\title{
Secondary metabolites in fungal biotic interactions
}

\author{
Dissertation \\ to obtain the $\mathrm{Ph}$. D. degree \\ in the International Ph. D. Program for Agricultural Sciences in Goettingen (IPAG) \\ at the Faculty of Agricultural Sciences, \\ Georg-August-University Göttingen, Germany
}

\author{
Presented by \\ Yi Kuang
}

Born in Xi' an, P. R. China

Göttingen, March 2014 
1. Name of supervisor: Prof. Dr. Petr Karlovsky

2. Name of co-supervisor: Prof. Dr. Matthias Hahn

Date of dissertation: 09.05.2014 


\section{Contents}

Chapter 1: General introduction .........................................................................1

Fungal secondary metabolites ....................................................................... 1

Secondary metabolites in fungus-microbe interactions ....................................... 1

Secondary metabolites in fungus-plant interactions ........................................6

Secondary metabolites of Fusarium verticillioides ............................................ 9

Secondary metabolites of Gliocladium roseum ….............................................13

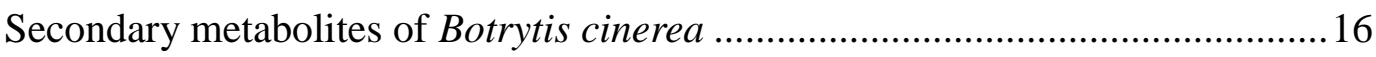

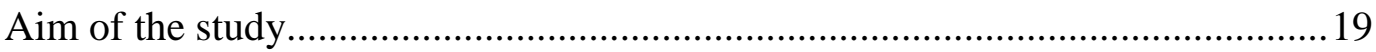

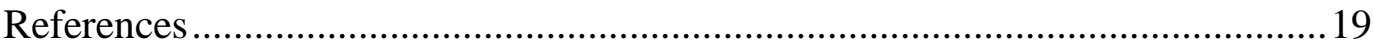

Chapter 2: Interactions among the fungi Aspergillus niger, Fusarium verticillioides, and Gliocladium roseum: effects on fungal biomass, metabolic

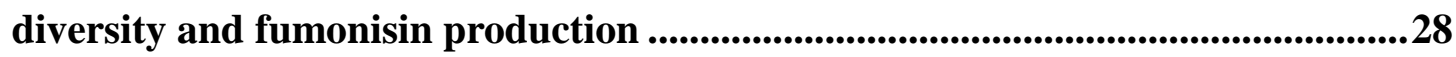

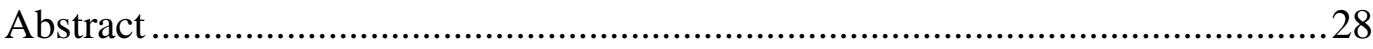

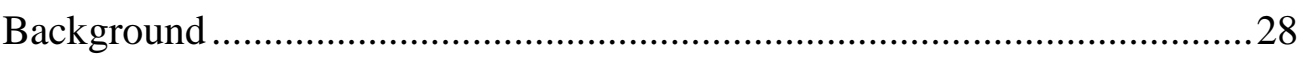

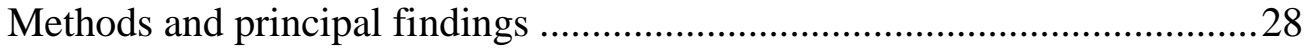

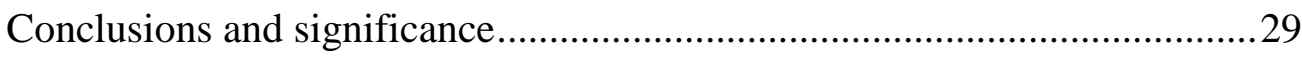

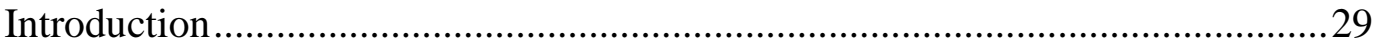

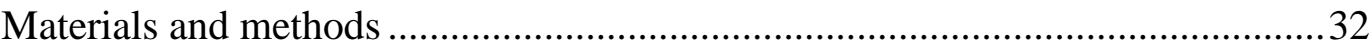

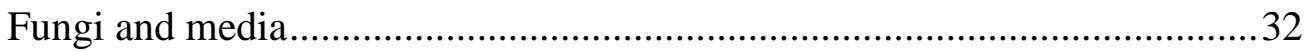

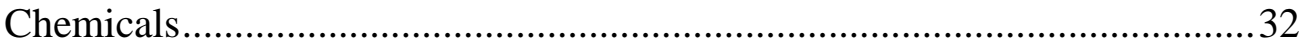

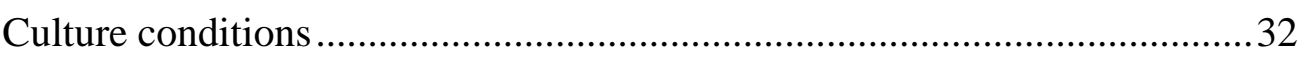

Determination of fungal biomass in dual cultures by densitometry of

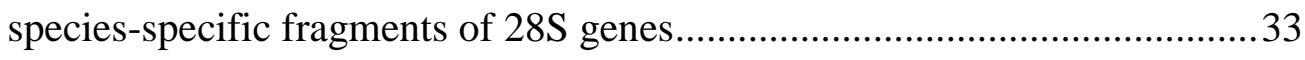

Estimation of the effect of competition on fitness .......................................35

Metabolic profiling of secreted fungal metabolites by HPLC-ESI-MS..........36

Determination of the content of fumonisins ..................................................38

Qualitative data analysis and descriptive statistics ......................................39 
Results. 39

Fungal biomass in dual cultures 39

Diversity of secreted metabolites 40

Suppression and stimulation of metabolite production by fungal interactions 42

Fumonisin synthesis in dual cultures 46

Degradation of FB1 by G. roseum 47

Discussion 48

Competitive fitness 48

Metabolic diversity

Stimulation, suppression, and degradation of secondary metabolites in dual cultures 50

Inhibition of fumonisin synthesis in F. verticillioides by G. roseum 51

References 53

Chapter 3: Production and antifungal activity of fusaric acid derivatives from Fusarium verticillioides co-cultured with Gliocladium roseum .57

Abstract 57

Introduction 58

Materials and methods 60

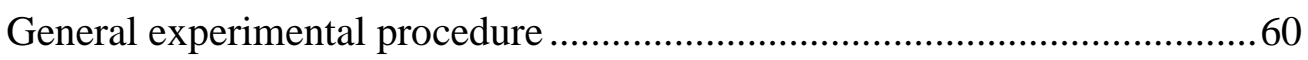

Chemicals 60

Fungal strains 60

Culture conditions .60

Metabolic profiling by HPLC-ESI-MS ...................................................61

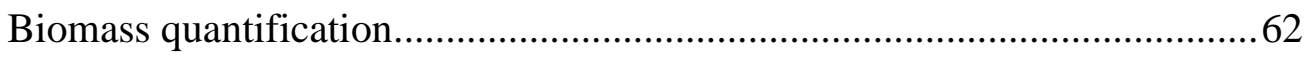

Analysis of fusaric acid (FA) by HPLC-DAD ..........................................63

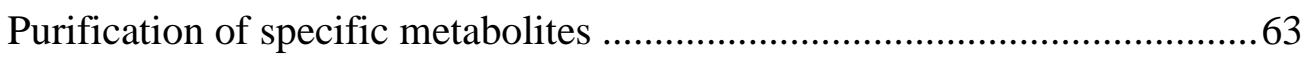

Interactions of $F$. verticillioides or $G$. roseum with other fungal species ......64

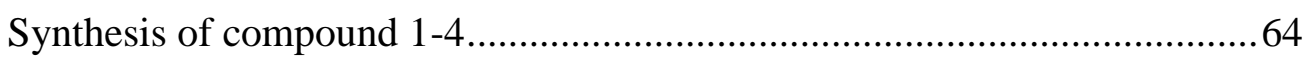

FA biotransformation 67 


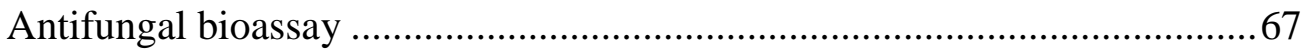

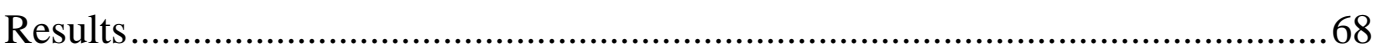

Secondary metabolites produced by mixed cultures....................................68

Identification of interaction-specific metabolites ......................................69

Accumulation of FA and fungal biomass in co-cultures of $F$. verticillioides

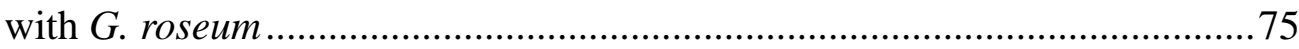

Origin of FA derivatives and accumulation of FA in co-cultures of F. verticillioides with different fungi ........................................................75

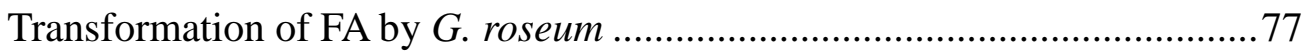

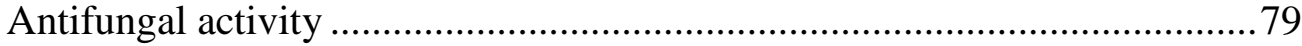

Degradation of FA by G. flavor-fuscum, G. catenulatum and T. harzianum .79

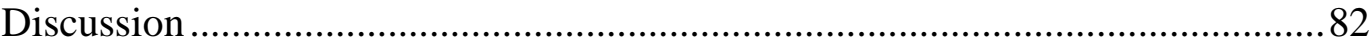

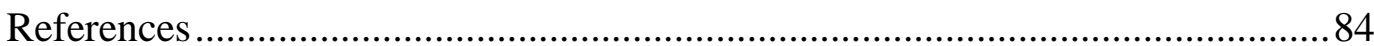

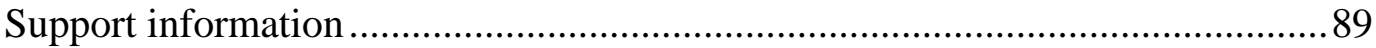

Chapter 4: LC-MS based metabolic profiling of Botrytis species...........................100

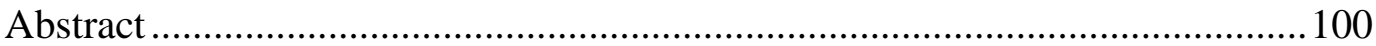

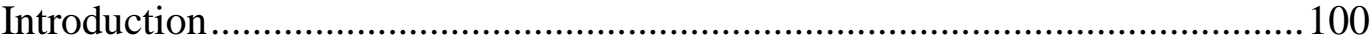

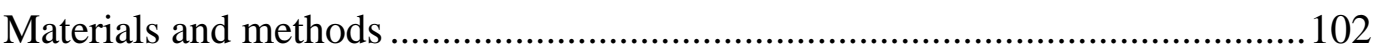

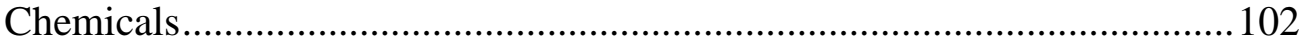

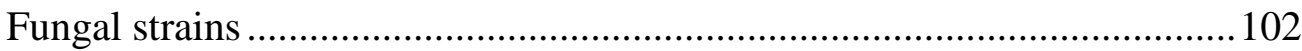

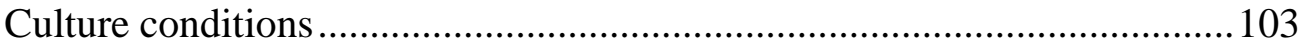

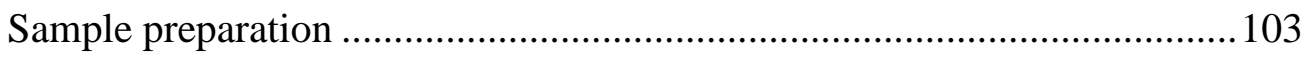

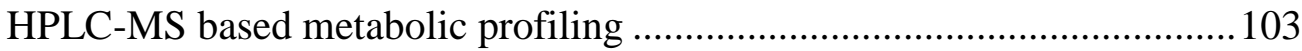

Data processing and statistical analysis .................................................. 104

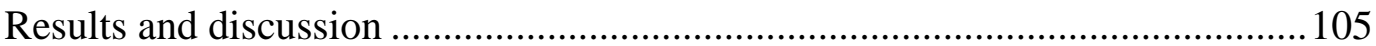

Comparison of metabolites produced by different Botrytis strains .............105

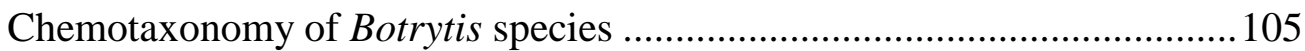

Comparison of metabolites produced by Botrytis cinerea $\mathrm{B} 05.10$ and its three

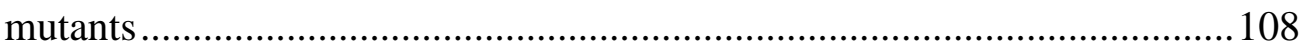

Comparison of Botrytis cinerea biomass ................................................. 111 


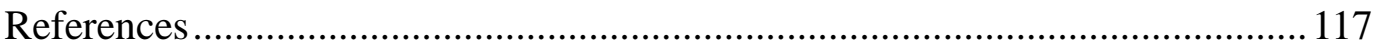

Chapter 5: General discussion ..........................................................................................121

Secondary metabolites in fungal interaction.................................................121

Metabolic profiling approaches in fungal secondary metabolites ..................... 124

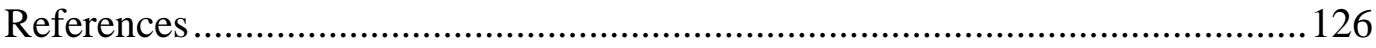

Summary

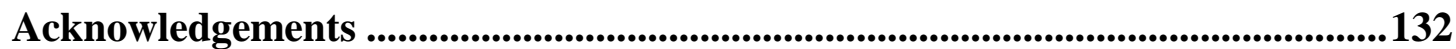

Curriculum vitae ..................................................................................................................134

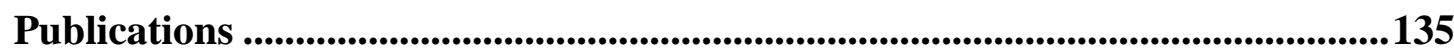




\section{Chapter 1: General introduction}

\section{Fungal secondary metabolites}

Fungi are non-photosynthetic microorganisms and play a key role in different environments. They do not live in isolation but occur in complex biotic ecosystems with other organisms like microbes, insects or plants. Fungi can be classified as pathogens or saprophytes. Pathogens attack living plants, insects or mammals while saprophytes grow on dead material (Hanson, 2008). The filamentous fungi which have different kinds of hyphal networks are very important for the litter decomposition, biogeochemical cycling and also play an important role in soil tilth and structure (Klein and Paschke, 2004).

Secondary metabolites, unlike primary metabolites, are not directly involved in the normal growth, development, or reproduction of an organism. Fungi are known for their production of chemical and biological diversity of secondary metabolites, containing polyketides, terpenoid metabolites, steroids, indole alkaloids and peptides. These secondary metabolites show diverse biological activities including phytotoxic, antibacterial, antifungal and antitumor activities.

To understand the structure, chemistry and biological activity of fungal secondary metabolites has made contributions to the development of natural product chemistry (Hanson, 2008).

\section{Secondary metabolites in fungus-microbe interactions}

In the modern natural product chemistry, cultivation of pure strain microorganisms is considered to be a standard method for the metabolites purification. During last decades, secondary metabolites isolated from the single-strain cultures have being studied extensively. However, the growth conditions of the single-strain cultures are significantly different from the natural environment where the microorganisms are interacting with each other (Onaka et al., 2011). To overcome this limitation, many new fermentation methods have been developed, one of them is co-cultivation of two or more microbes together (Zuck et al., 2011). 
Interactions among microbes lead to the activation of complex regulation mechanisms, which encompass antagonistic or competitive, facilitative and neutral outcomes (Rodriguez Estrada et al., 2011). Several investigations into natural products isolated from co-culture experiments indicated that the microbial interactions could be the reason for the production of necessary bioactive secondary metabolites. Therefore, co-culturing different microbial strains can be used as an effective method to harvest new molecules. A new antibiotic, pestalone (1), was first isolated from the mixed fermentation culture of a marine deuteromycete Pestalotia sp. with a marine bacterium, which could not be detected when either strain was cultured separately. Pestalone displayed potent antibacterial activity against methillin-resistant Staphylococcus aureus and vancomycin-resistant Enterococcus faecium, suggesting this new compound could be evaluated inadvaced models of infectious disease (Cueto et al., 2001).<smiles>COc1c(Cl)c(C)c(Cl)c(O)c1C(=O)c1c(O)cc(O)c(CC=C(C)C)c1C(C)C</smiles>

In the same year, Li et al. (2011) reported the isolation of a new xanthone derivative, 8-hydroxy-3-methyl-9-oxo-9H-xanthene-1-carboxylic acid methyl ether (2) from the co-culture broth of two marine fungi (strain No. E33 and K38) and could not be detected when either strain cultured alone. This xanthone derivative showed inhibitory activity against five microorganisms, including Gloeasporium musae and Peronophthora cichoralearum.<smiles>COC(=O)c1cc(C)cc2oc3cccc(O)c3c(=O)c12</smiles> 
Oh et al. (2007) reported that the co-cultivation of the marine-derived fungus Emericella sp. and the marine actinomycete Salinispora arenicola strongly induce the production of two new antimicrobial cyclic depsipeptides, emericellamides A and B (3 and 4). Emericellamides $A$ and $B$ showed moderate antimicrobial activity against methicillin-resistant Staphylococcus aureus and low cytotoxicity against HCT-116 human colon carcinoma cell line.

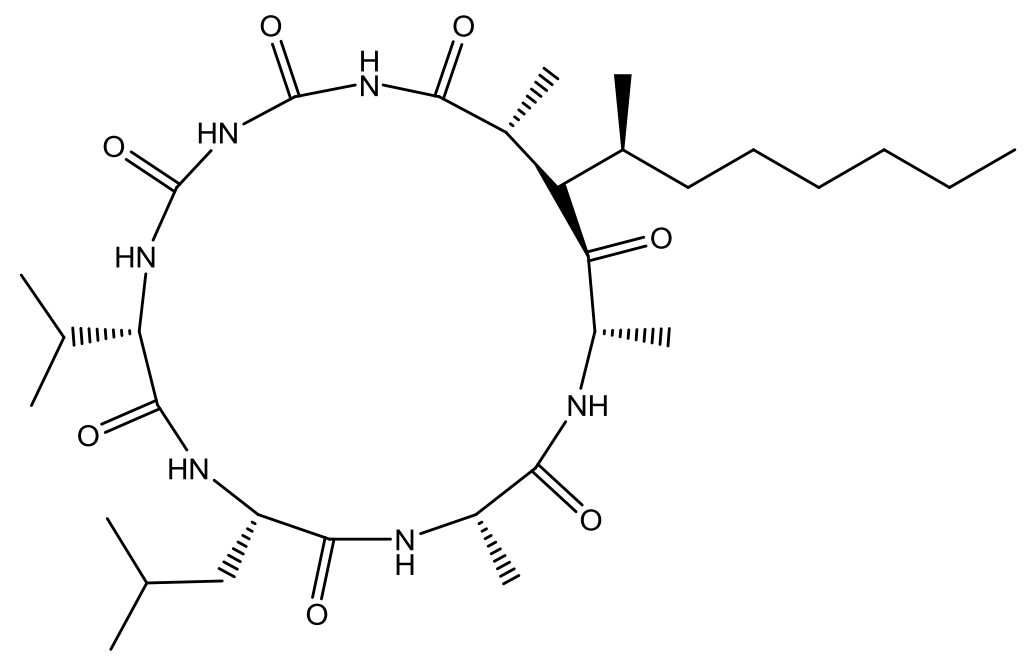

3

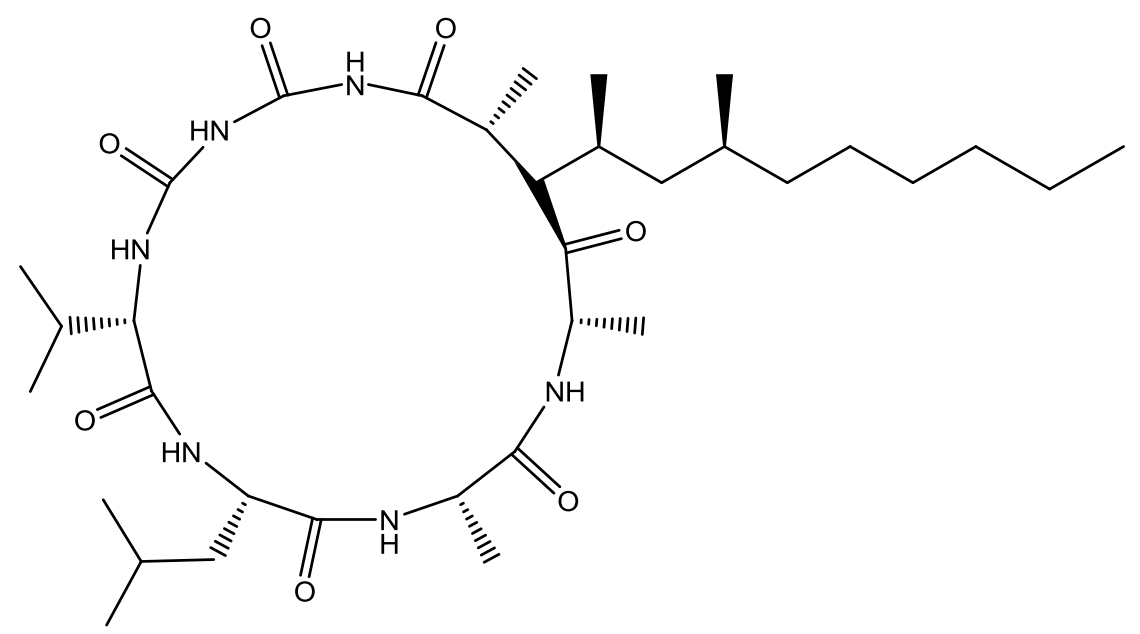

4

Recently, another two new metabolites, fumiformamide (5) and N, N'-((1Z, 3Z)-1, 4-bis (4-methoxyphenyl) buta-1, 3-diene-2, 3-diyl) diformamide (6) together with two known $N$-formyl derivatives and a xanthocillin analogue BU-4704 were obtained from the mix culture of the fungus Aspergillus fumigatus with the bacteria 
Streptomyces peucetius (Zuck et al., 2011).<smiles>COc1ccc(/C=C(NC=O)/C(=C/c2ccc(OS(=O)(=O)O)cc2)NC=O)cc1</smiles><smiles>COc1ccc(C=C(NC=O)/C(=C/c2ccc(OC)cc2)NC=O)cc1</smiles>

6

Another research on the mix fermentation successfully identified new metabolite as 4"-hydroxysulfoxy-2, 2"-dimethylthielavin P (7) in co-culture of Trichophyton rubrum and Bionectria ochroleuca. 4"-hydroxysulfoxy-2, 2"-dimethylthielavin P is a substitute of compound (8) found in the pure strain culture of B. ochroleuca. They suggested that B. ochroleuca produced $\mathbf{8}$, then $\mathbf{8}$ was further sulfated into $\mathbf{7}$ in the fungal interaction culture (Bertrand et al., 2013).<smiles>COc1c(C)c(OC(=O)c2c(C)c(C)c(OC(=O)c3c(C)c(C)c(OC(=O)O)c(C)c3OC)c(C)c2O)c(C)c(C(=O)O)c1C</smiles>

7. $\mathrm{R}=\mathrm{SO}_{3}, \quad 8 . \mathrm{R}=\mathrm{H}$

Schroeckh et al. (2009) reported that intimate physical interaction between 
Aspergillus nidulans and 58 soil-dwelling bacteria indicated that fungal-bacterial interaction could activate silent fungal metabolism genes at the molecular level (Schroeckh et al., 2009). In 2011, the same group, demonstrated that the Saga/Ada complex containing the protein (HAT GenE and AdaB) which is required for induction of the orsellinic acid gene cluster by the bacterium Streptomyces rapamycinicus, and Saga/Ada-dependent increase of histone 3 acetylation at lysine 9 and 14 occurs during interaction of Aspergillus nidulans and bacterium Streptomyces rapamycinicus. Their work provided insight into bacteria-triggered histone modification in a fungus as potential basis for a crosstalk between different species of microorganisms (Nützmann et al., 2011). In 2013, one paper reported that the co-cultivation of the bacterium Streptomyces rapamycinicus with human pathogenic fungus Aspergillus fumigatus induced a silent polyketide synthase pathway which led to the discovery of a previously unreported prenylated polyketide (9) (König et al., 2013).<smiles>CC(=O)C=C1CC2(O)Cc3c(c(O)c4c(O)cc(O)cc4c3CC=C(C)C)C(=O)C2O1</smiles>

Recently, Fusarium tricinctum and F. begonia were co-cultivated together. Their co-cultivation could induced the production of two new linear depsipeptides, subenniatins A and B (10 and 11), which were not detectable when either of the two fungi was grown individually. The structure of subenniatins A and B are biogenetic building blocks of the enniatins $\mathrm{B}, \mathrm{B} 1, \mathrm{~A}$, and $\mathrm{A} 1$, which are mycotoxins produced by F. tricinctum alone (Wang et al., 2013).<smiles>[R]CC(C)[C@H](C(=O)O[C@H](CN(C)[C@H](C(=O)O)C(C)C)C(C)C)N(C)C(=O)[C@@H](O)C(C)C</smiles>

10. $\mathrm{R}=\mathrm{H}$

11. $\mathrm{R}=\mathrm{CH}_{3}$ 
Mycotoxin producing strains, interacting with other microbes could affect the mycotoxin production. Co-culture of Fusarium culmorum Fc13, F. graminearum Fg23 and two Alternaria tenuissima isolates (At18 and At220) on wheat kernels in vitro simultaneously or consecutively has been reported. The production of mycotoxin showed that the combination of A. tenuissima At18 and A. tenuissima At220 with F. graminearum $\mathrm{Fg} 23$ inhibited the toxin production of both fungal partners. In contrast, F. culmorum Fc13 increased its DON and ZON production in competitive interaction with both A. tenuissima strains (Müller et al., 2012). The interactions between fumonisin producer Fusarium verticillioides and Fusarium proliferatum and zearalenone- and deoxynivalenol-producing isolate of Fusarium graminearum inoculated together on irradiated maize. On artificially inoculated maize earsthe production of fumonisin B1 by $F$. verticillioides and $F$. proliferatum was always inhibited by the presence of $F$. graminearum; however, the production of deoxynivalenol by $F$. graminearum was significantly stimulated when paired with the fumonisin producer. The presence of $F$. graminearum decreased the fungal populations of $F$. verticillioides and $F$. proliferatum (Velluti et al., 2001).

Furthermore, in vitro experiments of the biomass of Ustilago maydis was drastically reduced in interaction with $F$. verticillioides (Rodriguez Estrada et al., 2011). In 2012, they clarified the potential mechanisms of antagonism between these two fungi that also may occur in the soil or in maize, niches for both fungi where they were likely interact in nature. The decline of biomass of $U$. maydis may be attributed to the secretion of mycotoxins and the expression of genes encoding adhesive and cell wall-degrading proteins by $F$. verticillioides. $U$. maydis responded to co-cultivation by expressing siderophore biosynthetic genes and more highly expressed genes potentially involved in toxin biosynthesis (Jonkers et al., 2012).

The co-cultivation represents a potentially important strategy for discovery of new bioactive metabolites, however, the mechanism of the fungal-microbial interaction which is thought to be caused by the secondary metabolites is still poorly understood.

\section{Secondary metabolites in fungus-plant interactions}

Plants provide a perfect ecosystem for microorganisms. The interactions among a wide variety of microbes with plants occur in all parts of the plant from the aerial 
parts to the root system (Montesinos et al., 2002). In modern agricultural practices, major diseases of crop plants are caused by plant pathogens leading to enormous threat to food security worldwide (Strange and Scott, 2005). The interactions between plants and diverse fungal species in complex symbiotic, parasitic and pathogenic pathways are the most important relationships among them. The phytopathogenic fungi are often the producer of phytotoxins. The effect of phytotoxins is characterized by symptoms, wilting and growth suppression on plants (Berestetskiy, 2008).

Phytotoxins are classified into host-specific and non-host-specific. Host-specific phytotoxins are only toxic to susceptible plants and have contributed to a great advance in research on plant-microbe interactions (Otani et al., 1995). The host-specific toxins act as a primary determinant of pathogenicity in establishing disease development, whereas a limited number of host-specific phytotoxins are known yet. Alternaria species are well known for the production of a range of host-specific toxins including AAL-, ACT-, AF-, AT-, AM-, AK-toxins (Otani et al., 1995). In the interactions of A. alternata and tomato, the host-specific AAL-toxin plays an important role in the pathogenicity of the fungus, leading to cell death in susceptible cultivars. Tomato plants of the genotype asc/asc are susceptible to AAL-toxin while genotype plants Asc/Asc are less sensitive to the AAL-toxin (Akamatsu et al., 1997). The AK-toxins (12 and 13), AF-toxins (14 and 15) and ACT-toxins (16 and 17) share a common structural moiety, which is 9, 10-epoxy-8-hydroxy-9-methyl-decatrienoic acid. AKT7 (AK-toxin biosynthetic gene 7), a new identified gene, in the interaction of A. alternata and Japanese pear functions to limit AK-toxin production (Takaoka et al., 2014). Destruxin B (18), produced by $A$. brassicae, reported as host-specific virulence factor cause gray leaf spot in Brassica plants (Pedras et al., 2002). In the investigation of the role of destruxin B in infection of pathogen, suggested that destruxin B is not a host-specific toxin and does not change the accessibility of its host that necessary for colonization of the pathogen (Parada et al., 2007).

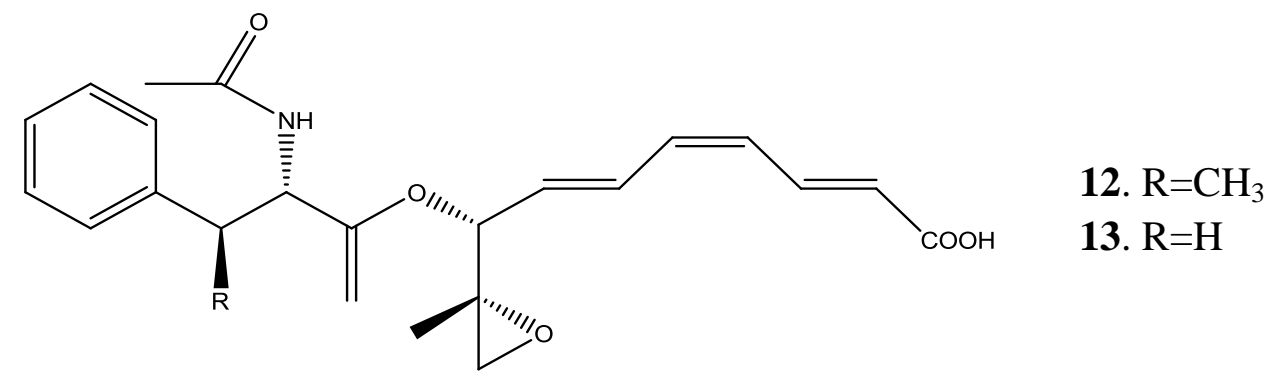



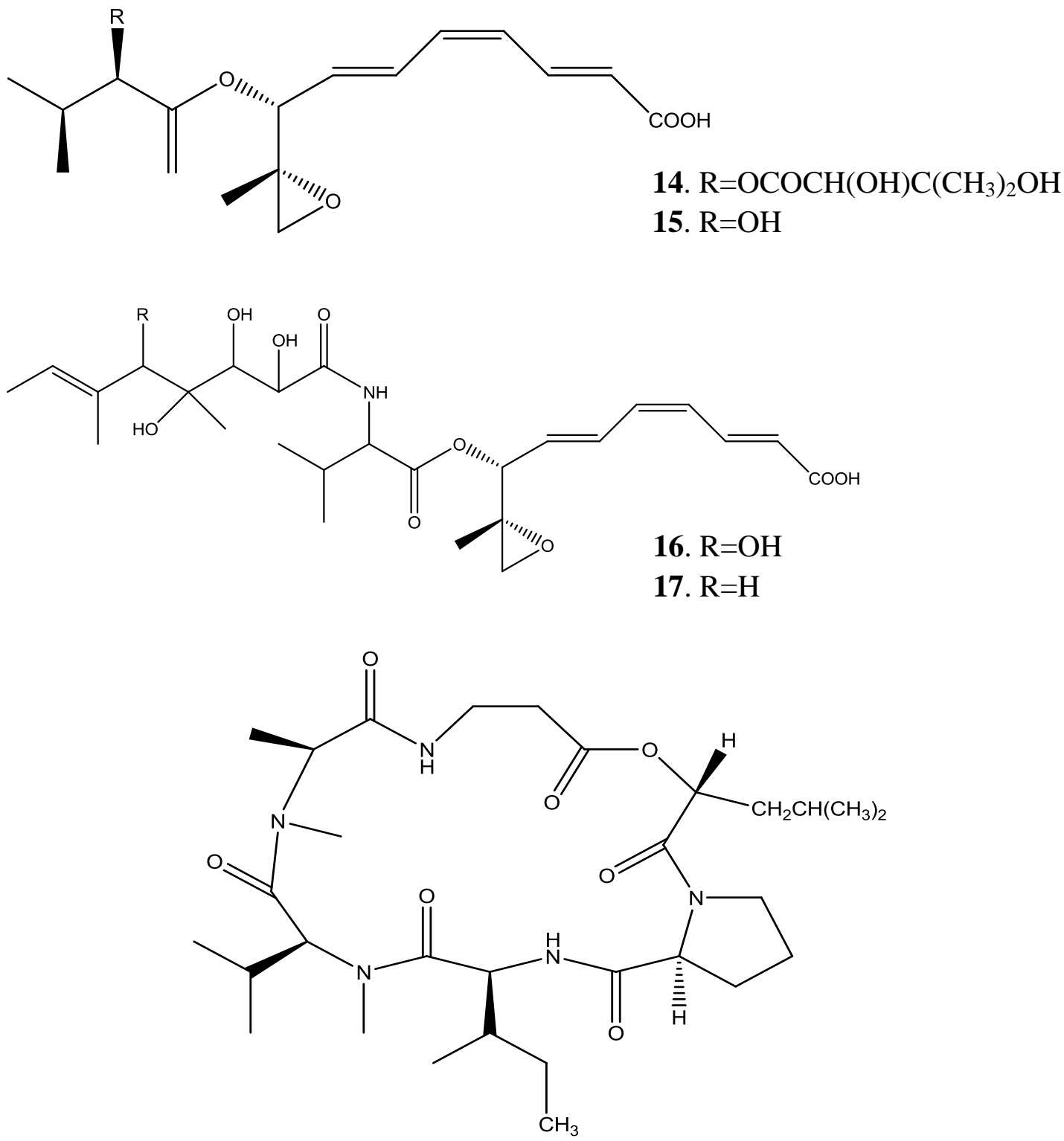

18

By far, the majority of phytotoxins are nonselective and do not reproduce the resistance and susceptibility of the host to the pathogen. F. graminearum, which is one of the most important pathogens of crops and causes Fusarium head blight (FHB) in wheat, barley and other small grain cereals, and Fusarium ear rot (FER) in maize (Boutigny et al., 2014). Trichothecenes are nonselective phytotoxins showing highly toxicity to wheat and rye (Nishiuchi et al., 2006). Jansen et al. (2005) reported that trichothecenes produced by $F$. graminearum, could act as virulence factors aiding the spread of the fungus on sensitive host plant during disease development. In the absence of trichothecenes, the fungus is blocked by the development of heavy cell wall thickenings in the rachis node of Nandu wheat. 
The phytotoxin ( $F v$ toxin) produced by Fusarium virguliforme caused sudden death syndrome disease of soybean. During its colonization of the roots and its translocation through the vascular system to the leaves causing interveinal chlorosis and necrosis leading to defoliation and early maturation (Radwan et al., 2013). While non-host-specific phytotoxins always play a role as virulence factors during plant colonization, host-specific phytotoxins act as pathogenicity factors in the plant-microbe interactions.

\section{Secondary metabolites of Fusarium verticillioides}

Fusarium verticillioides (Sacc. Nirenberg, syn. Moniliforme Sheldon), formerly known as Fusarium moniliforme, is the most important ear and kernel rotting pathogen of maize in most tropical and temperate regions, causing major damage and losses (Presello et al., 2008; Sanchez-Rangel et al., 2005). The typical F. verticillioides symptoms occur on single or small groups of kernels are as white or pink mold. There are several pathways $F$. verticillioides infects the maize cobs such as systemically from the seed or by infection through the silks (Munkvold et al., 1997). As a famous producer of biologically active secondary metabolites, F. verticillioides produced a set of toxic secondary metabolites including fumonisins, fusaric acid and fusarins (Brown et al., 2012).

Fumonisins (Figure 1) are a family of polyketide-derived natural products first isolated in 1988 from cultures of $F$. verticillioides (Gelderblom et al., 1988). Fumonisins have similar structure to sphinganine, and are known to disrupt the sphingolipid biosynthetic pathway (Wang et al., 1991). The biosynthesis of fumonisins requires a cluster of contiguous and co-regulated FUM genes (Proctor et al., 2003). This group of mycotoxin has been designated as A, B, C and P-series fumonisins. Fumonisin B is characterized by a 20 carbon backbone with an amine function, one to four hydroxyl functions, and two chains which are diesterified with propane-1, 2, 3-tricarballylic acid. The occurrence of fumonisins B in infected maize and maize products have been reported to cause variety of diseases in animals after eating the contaminated feeds, such as leukoencephalomalacia in horses and pulmonary edema syndrome in swine (Ross et al., 1990) and plays a role in human esophageal cancer and neural tube defects (Marasas et al., 2004). Control of fumonisin contamination in maize has become a priority in food safety research. 
Fumonisin B1, fumonisin B2 and fumonisin B3 are the most abundant fumonisins in nature which are mainly produced by F. verticillioides (Nelson et al., 1991; Sanchez-Rangel et al., 2005). FB1 typically accounts for $70-80 \%$ of the total fumonisins detected, followed by FB2 with 15-25\% and FB3 with 3-8\% (Branham and Plattner, 1993; Marin et al., 1995). Fumonisin A and C series typically occur in lower concentrations than fumonisin B. Fumonisin C analogues differ in structure from the B-series as their backbone consists of 19 carbons chain. Fumonisin A analogues differ in their structure from the B-series due to the acetylation of the terminal amine group (Bartók et al., 2006). The structures of fumonisin P analogues are identical to fumonisin B, only the amine function group was replaced with an N-linked 3-hydroxypiridinium moiety. Fumonisin $\mathrm{P}$ have been found on F. verticillioides grown on cracked maize kernel medium when the production of FB1 were at levels up to $30 \%$ (Musser et al., 1996). Fumonisin C and fumonisin P have been found to be phytotoxic to duckweed and cytotoxic to mammalian cell lines H4TG and MDCK (Abbas et al., 1998).

Fusaric acid (5-butylpicolinic acid) is a phytotoxin which was first reported in 1934 produced by Gibberella fujikuroi on rice plants (Yabuta et al., 1934). Its molecular structure (Figure 2) has been reported to exist in F. moniliforme (Burmeister et al., 1985). As a plant toxin fusaric acid is associated with damping-off and root rot diseases of many vegetables and crops while it is considered as a mild toxin to the mammals and brine shrimp (Capasso et al., 1996).

The fusarins (Figure 3) are a group of polyketide compounds which have a 3-pyrolidone ring and pentaene chain. It has been reported that the pentaene chain can easily rearrange under UV light, forming several stereoisomers like (10Z)-, (8Z)-, and (6Z)-fusarin C (Kleigrewe et al., 2012). Fusarin A and D are non-mutagenic, therefore, fusarin C was concentrated in most of fusarin studies (Savard and Miller, 1992). Fusarin C has been reported to be mutagenic in Ames Salmonella mutagenicity assay (Gelderblom et al., 1983) and it could act as an estrogenic mimic which stimulated growth of a breast cancer cell (Sondergaard et al., 2011). 
<smiles>[R]C(CCCCC([R2])CC(O)C(C)N)CC(C)CC(OC(=O)CC(CC(=O)O)C(=O)O)C(OC(=O)CC(CC(=O)O)C(=O)O)C(C)CCCC</smiles><smiles>[R]C(CCCCC([R])CC(O)C(C)NC(C)=O)CC(C)CC(OC(=O)CC(CC(=O)O)C(=O)O)C(OC(=O)CC(CC(=O)O)C(=O)O)C(C)CCCC</smiles>

$\begin{array}{llr} & \mathrm{R}_{1} & \mathrm{R}_{2} \\ \text { FA1 } & \mathrm{OH} & \mathrm{OH} \\ \text { FA2 } & \mathrm{OH} & \mathrm{H} \\ \text { FA3 } & \mathrm{H} & \mathrm{OH}\end{array}$<smiles>[R]C(CCCCC([R])CC(O)CN)CC(C)CC(OC(=O)CC(CC(=O)O)C(=O)O)C(OC(=O)CC(CC(=O)O)C(=O)O)C(C)CCCC</smiles>

$\mathrm{FB} 1 \mathrm{OH} \quad \mathrm{OH}$

FB2 $\mathrm{H} \quad \mathrm{OH}$

FB3 $\mathrm{OH} \quad \mathrm{H}$

FB4 $\mathrm{H} \quad \mathrm{H}$

FB5 $\mathrm{OH} \quad \mathrm{H}$

FA3 $\mathrm{H} \quad \mathrm{OH}$<smiles>[R7]C(CCCCC([R])CC(O)C(C)[n+]1cccc(O)c1)CC(C)CC(OC(=O)CC(CC(=O)O)C(=O)O)C(OC(=O)CC(CC(=O)O)C(=O)O)C(C)CCCC</smiles>

$\mathrm{R}_{1} \quad \mathrm{R}_{2}$ FP1 $\mathrm{OH} \quad \mathrm{OH}$ FP2 OH $\mathrm{H}$ FP3 $\mathrm{H} \quad \mathrm{OH}$

Figure 1. Chemical structures of the major fumonisin series 
<smiles>CCCCc1ccc(C(=O)O)nc1</smiles>

Figure 2. Chemical structure of fusaric acid<smiles>[R][C@]1(C(=O)/C(C)=C/C=C/C(C)=C/C(C)=C/C(=C\C)C(=O)OC)C(=O)N[C@]2(O)CCO[C@H]21</smiles>

Fusarin D $\quad \mathrm{R}=\mathrm{OH}$

Fusarin A $\mathrm{R}=\mathrm{H}$<smiles>C/C=C(\C=C(C)\C=C(C)\C=C\C=C(/C)C(=O)[C@@]12O[C@H]1[C@@](O)(CCO)NC2=O)C(=O)OC</smiles>

Fusarin C<smiles>C/C=C(\C=C(C)\C=C(C)\C=C\C=C(/C)C(=O)[C@@]1(O)C(=O)N[C@]2(CCO)O[C@H]21)C(=O)OC</smiles>

Fusarin F

Figure 3. Chemical structures of major fusarins 


\section{Secondary metabolites of Gliocladium roseum}

Fungi of the Gliocladium genus are commonly used as biological control agents against plant pathogenic fungi, such as Pythium ultimum and Rhizoctonia solani. The fungus Gliocladium roseum is widely distributed in different environments (tropical, temperate, desert). G. roseum could associate with other organisms such as fungi, several nematodes and the roots, stems, leaves, fruits, seeds of plants (Sutton et al., 1997; Zhang et al., 2008). As a destructive mycoparasite, it is a common facultative saprophyte in the soil (Schroers et al., 1999), which has been tested successfully as a biological control agent against several plant pathogenic fungi including Botrytis spp., and Fusarium spp. (Li et al., 2002; Xue et al., 2002). From light microscopy showed that the hyphae of G. roseum can parasitize B. cinerea (Yu and Sutton, 1997). The mycoparasitic activity has been attributed to the production of the cell wall degrading enzymes, including chitinase and $\beta-(1,3)$-glucanase, and its production of some toxins or antifungal substances (Pachenari and Dix, 1980; Li et al., 2002). Utermark and Karlovsky (2007) reported that G. roseum could be used as a biological control agent due to its ability to detoxify the mycotoxin zearalenone by producing a zearalenone-specific lactonase which catalyzes the hydrolysis of zearalenone, followed by a spontaneous decarboxylation. Phytochemical studies undertaken by different groups have resulted in the isolation of various compounds which shown various bioactivities, including diketopiperazines, terpenoids, peptides and polyketides (Dong et al., 2005; Bräse et al., 2009). Four polyterpenoids glisoprenins have been isolated from the cultures of G. roseum HA190-95 (Figure 4). And the glisoprenins C, D and E could be used as inhibitors of appressorium formation in Magnaporthe grisea (Thines et al., 1997; Sterner et al., 1998).

In 1999, roselipins (Figure 5) were isolated as inhibitors of diacylglycerol acyltransferase from cultures of G. roseum KF-1040 (Noriko et al., 1999). Dong et al. (2005) isolated five new verticillin-type epipolysulfanyldioxopiperazines (gliocladines A-E) along with four known compounds verticillin A, 11'-deoxyverticillin A, Sch52900 and Sch52901 from G. roseum 1A using solid-substrate fermentation (Figure 6). All these nine metabolites showed antinematodal activities against Caenorhabditis elegans and Panagrellus redivivus. Furthermore, a series of volatile hydrocarbons and hydrocarbon derivatives on an 
oatmeal-based agar under microaerophilic conditions have been reported to be produced by G. roseum NRRL 50072 (Strobel et al., 2008).<smiles>CC(C)=CCCC(C)(O)CCCC(C)(O)CCCC(C)(O)CCCC(C)=CCCC(C)=CCCC(C)=CCCC(C)=CCCC(C)(O)CCCO</smiles>

Glisoprenin A<smiles>CC(=CCCC1(C)OC1CCC(C)=CCO)CCC=C(C)CCCC(C)(O)CCCC(C)(O)CCCC(C)(O)CCCC(C)(O)CCCC(C)(O)O</smiles>

Glisoprenin B<smiles>CC(=CCO)CCC=C(C)CCC=C(C)CCC=C(C)CCCC(C)(O)CCCC(C)(O)CCCC(C)(O)CCCC(C)(O)C(C)(C)O</smiles>

Glisoprenin C<smiles>CC(=CCCC(C)(O)C(O)CCC(C)=CCO)CCC=C(C)CCCC(C)(O)CCCC(C)(O)CCCC(C)(O)CCCC(C)(O)CCCC(O)(O)C(C)(C)O</smiles>

Figure 4. Chemical structures of glisoprenins A-D 


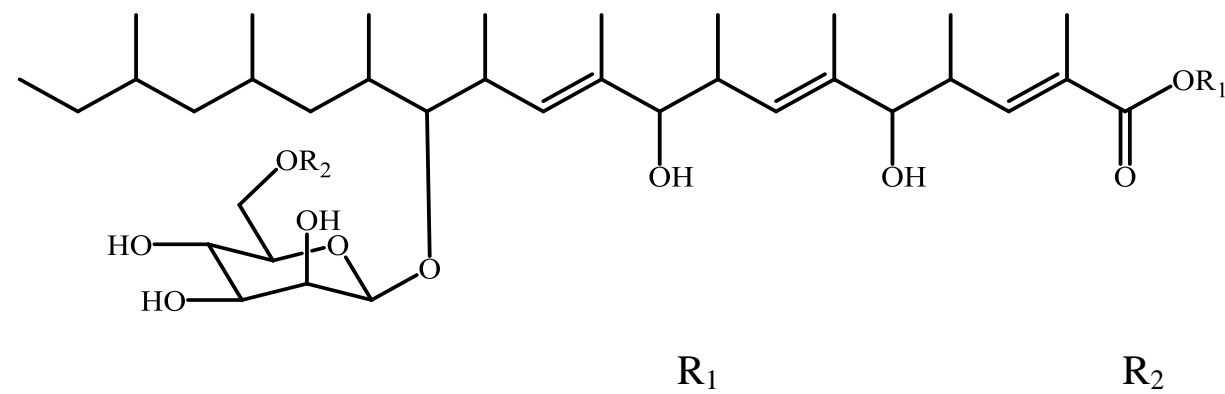

Roselipin 1A<smiles>CC[C@H](O)[C@@H](O)[C@H](O)CO</smiles>

$\mathrm{H}$

Roselipin 1B<smiles>CC[C@H](O)[C@@H](O)[C@H](O)CO</smiles>

Roselipin 2A<smiles>CC[C@H](O)[C@@H](O)[C@H](O)CO</smiles>

$\mathrm{COCH}_{3}$

Roselipin 2B<smiles>CC[C@H](O)[C@@H](O)[C@H](O)CO</smiles>

$\mathrm{COCH}_{3}$

Figure 5. Chemical structures of roselipins 


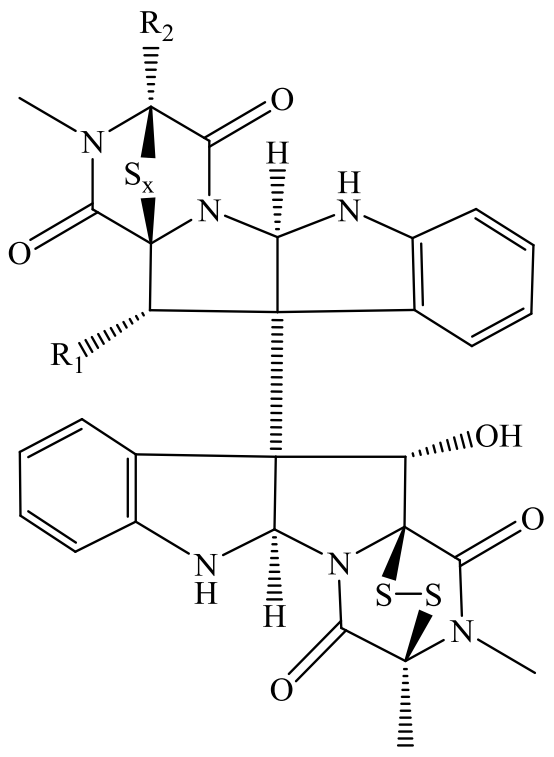<smiles>[3H][C@]12Nc3ccccc3[C@]1(c1c[nH]c3ccccc13)[C@@H](O)[C@]13S[C@](C)(C(=O)N12)N(C)C3=O</smiles>

Gliocladine $\mathrm{A} \quad \mathrm{R}_{1}=\mathrm{OH} \quad \mathrm{R}_{2}=\mathrm{CH}_{3} \quad \mathrm{X}=3$

Gliocladine $\mathrm{B} \quad \mathrm{R}_{1}=\mathrm{OH} \quad \mathrm{R}_{2}=\mathrm{CH}_{3} \quad \mathrm{X}=4$

Verticillin A $\quad \mathrm{R}_{1}=\mathrm{OH} \quad \mathrm{R}_{2}=\mathrm{CH}_{3} \quad \mathrm{X}=2$

11'-deoxyverticillin A $\quad \mathrm{R}_{1}=\mathrm{H} \quad \mathrm{R}_{2}=\mathrm{CH}_{3} \quad \mathrm{X}=2$

Sch52900 $\quad \mathrm{R}_{1}=\mathrm{OH} \quad \mathrm{R}_{2}=\mathrm{CH}_{2} \mathrm{CH}_{3} \quad \mathrm{X}=2$

$\operatorname{Sch} 52901 \quad \mathrm{R}_{1}=\mathrm{OH} \quad \mathrm{R}_{2}=\mathrm{CH}(\mathrm{OH}) \mathrm{CH}_{3} \quad \mathrm{X}=2$

Gliocladine C $\quad \mathrm{X}=2$

Gliocladine D $\quad X=3$

Gliocladine $\mathrm{E} \quad \mathrm{X}=4$

Figure 6. Chemical structures of verticillin-type compounds from G. roseum 1A

\section{Secondary metabolites of Botrytis cinerea}

Fungi from the genus Botrytis are fungi imperfecti belonging to the order Moniliales of the family Moniliaceae. Botrytis cinerea Pers.:Fr. [teleomorph Botryotinia fuckeliana (de Bary) Whetzel] is considered to be one of the top ten fungal plant pathogen listed for molecular plant pathology (Dean et al., 2012). B. cinerea is a necrotrophic plant pathogenic fungus which grows as grey mould in more than 200 commercial crops worldwide including grapes, lettuce, tomatoes, tobacco and strawberries and causing serious economic losses (Washington, 1993). The costs for chemical control of Botrytis represent $10 \%$ of the world fungicide market (UIPP, 2002), and the increasing problem of fungicide resistance has been reported recently (Leroch et al., 2011). B. cinerea is a well-known producer of many structurally 
diverse metabolites and some of these metabolites show phytotoxic activity (Figure 7).

A series of metabolites of sesquiterpenoid botryanes have been isolated from B. cinerea (Collado et al., 2007). The best known botryanes are botrydial, dihydrobotrydial and their derivatives which are responsible for the typical lesions caused by fungal infection (Fehlhaber et al., 1974). Another series of phytotoxic metabolites are polyketide lactones known as botrylactone (Welmar et al., 1979) and botcinolides (Jacyno et al., 1994). Botcinolide is a highly oxidized nine-membered lactone esterified with 4-hydroxy-2-octenoic acid which significantly inhibited etiolated wheat coleoptiles. During the following years, several botcinolide derivatives were found (Shiina and Fukui, 2009). Tani et al. (2005) isolated four metabolites botcinis A-D from B. cinerea, which showed antifungal activity against Magnaporthe grisea, a pathogen of rice blast. Later, they purified some other new botcinin analogs, such as botcinis E-F and botcinic acid from B. cinerea (Tani et al., 2006).

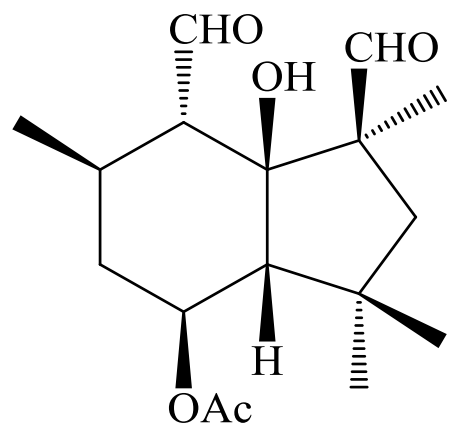

Botrydial

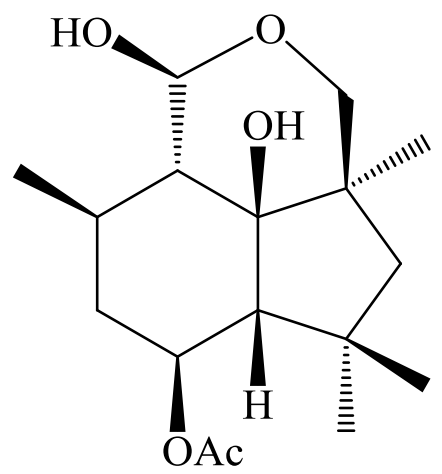

Dihyrobotrydial

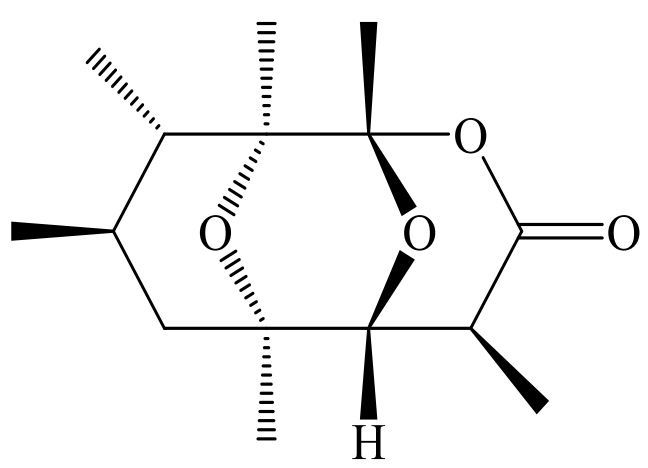

Botrylactone 


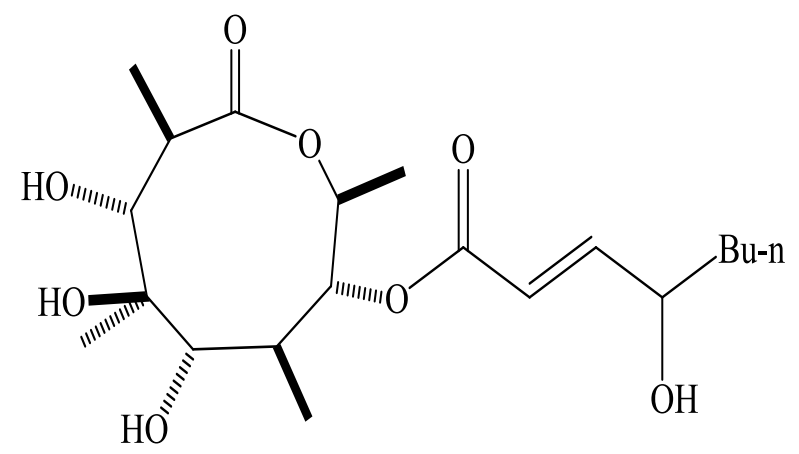

Botcinolide

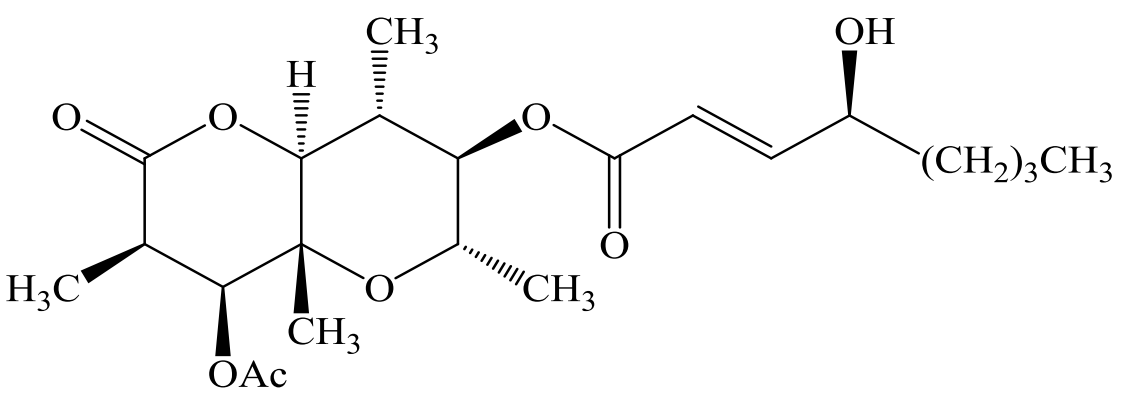

Botcin A<smiles>[R2]C1([R2])C(=O)O[C@H]2[C@@H](C)[C@@H](OC(=O)/C=C/[C@@H](O)CCC)[C@H](C)O[C@]2(C)[C@H]1OC(C)=O</smiles>

Botcin $\mathrm{B} \mathrm{R}_{1}=\mathrm{CH}_{3}, \mathrm{R}_{2}=\mathrm{H} ; \quad$ Botcin $\mathrm{C} \mathrm{R}_{1}=\mathrm{H}, \mathrm{R}_{2}=\mathrm{CH}_{3}$

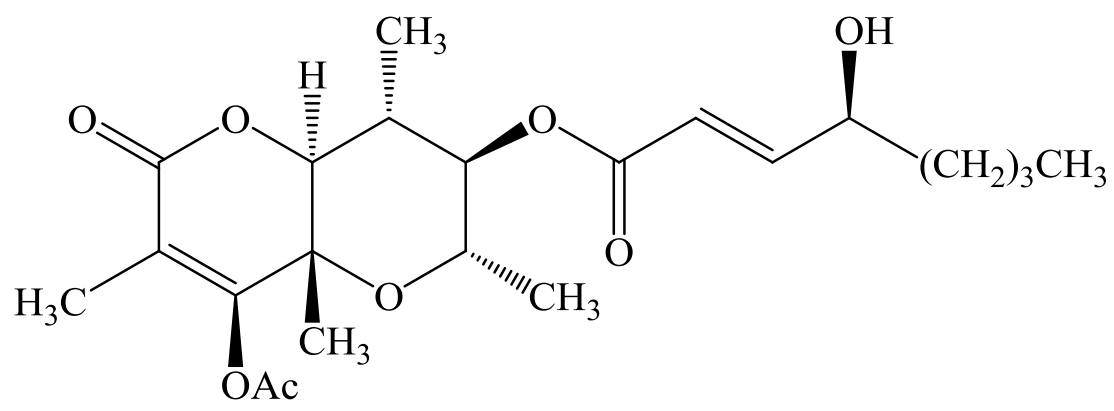

Botcin D 


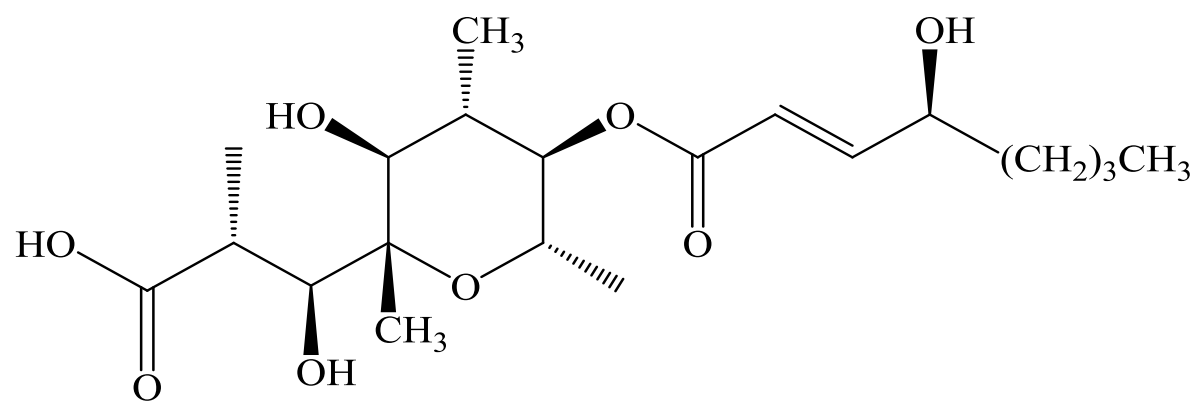

Botcinic acid

Figure 7. Chemical structures of some Botrytis cinerea metabolites

\section{Aim of the study}

Two major purposes were carried out for this study:

1. To investigate the role of secondary metabolites involved in the interaction between the phytopathogenic fungus Fusarium verticillioides and the mycoparastic fungus Gliocladium roseum.

2. To elucidate the chemical diversity in secondary metabolites production by Botrytis spp. and its potential role in host plant specialization.

\section{References}

Abbas, H.K., Shier, W.T., Seo, J.A., Lee, Y.W., Musser, S.M., 1998. Phytotoxicity and cytotoxicity of the fumonisin $\mathrm{C}$ and $\mathrm{P}$ series of mycotoxins from Fusarium spp. fungi. Toxicon 36, 2033-2037.

Akamatsu, H., Itoh, Y., Kodama, M., Otani, H., Kohmoto, K., 1997. AAL-toxin-deficient mutants of Alternaria alternata tomato pathotype by restriction enzyme-mediated integration. Phytopathology 87, 967-972.

Bartók, T., Szécsi, Á., Szekeres, A., Mesterházy, Á., Bartók, M., 2006. Detection of new fumonisin mycotoxins and fumonisin-like compounds by reversed-phase 
high-performance liquid chromatography/electrospray ionization ion trap mass spectrometry. Rapid Commun. Mass Spectrum 20, 2447-2462.

Berestetskiy, A.O., 2008. A review of fungal phytotoxins: from basic studies to practical use. Appl. Biochem. Microbiol. 44, 453-465.

Bertrand, S., Schumpp, O., Bohni, N., Monod, M., Gindro, K., Wolfender, J.L., 2013. De novo production of metabolites by fungal co-culture of Trichophyton rubrum and Bionectria ochroleuca. J. Nat. Prod. 76, 1157-1165.

Boutigny, A.L., Ward, T., Ballois, N., Iancu, G., Ioos, R., 2014. Diversity of the Fusarium graminearum species complex on French cereals. Eur. J. Plant Pathol. 138, 133-148.

Branham, B.E., Plattner, R.D., 1993. Alanine is a precursor in the biosynthesis of fumonisin B1 by Fusarium moniliforme. Mycopathologia 124, 99-104.

Bräse, S., Encinas, A., Keck, J., Nising, C.F., 2009. Chemistry and biology of mycotoxins and related fungal metabolites. Chem. Rev. 109, 3903-3990.

Brown, D.W., Butchko, R.A.E., Busman, M., Proctor, R.H., 2012. Identification of gene clusters associated with fusaric acid, fusarin, and perithecial pigment production in Fusarium verticillioides. Fungal Genet. Biol. 49, 521-532.

Burmeister, H.R., Grove, M.D., Peterson, R.E., Weisleder, D., Plattner, R.D., 1985. Isolation and characterization of two new fusaric acid analogs from Fusarium moniliforme NRRL13,163. Appl. Environ. Microbiol.50, 311-314.

Capasso, R., Evidente, A., Cutignano, A., Vurro, M., Zonno, M.C., Bottalico, A., 1996. Fusaric and 9, 10-dehydrofusaric acids and their methyl esters from Fusarium nygamai. Phytochemistry 41, 1035-1039.

Collado, I.G., Sanchez, A.J.M., Hanson, J.R., 2007. Fungal terpene metabolites: biosynthetic relationships and the control of the phytopathogenic fungus Botrytis cinerea. Nat. Prod. Rep. 24, 674-686.

Cueto, M., Jensen, P.R., Kauffman, C., Fenical, W., Lobkovsky, E., Clardy, J., 2001. Pestalone, a new antibiotic produced by a marine fungus in response to bacterial challenge. J. Nat. Prod. 64, 1444-1446.

Dean, R., Van Kan, J.A.L., Pretorius, Z.A., Hammond-Kosack, K.E., Di Pietro, A., 
Spanu, P.D., Rudd, J.J., Dickman, M., Kahmann, R., Ellis, J., Foster, G.D., 2012. The top 10 fungal pathogens in molecular plant pathology. Mol. Plant Pathol. 13, 414-430.

Dong, J.Y., He, H.P., Shen, Y.M., Zhang, K.Q., 2005. Nematicidal epipolysulfanyldioxopiperazines from Gliocladium roseum. J. Nat. Prod. 68, $1510-1513$.

Fehlhaber, H.W., Geipel, R., Mercker, H.J., Tschesche, R., Welmar, K., Schönbeck, F., 1974. Botrydial, ein sesquiterpen-antibiotikum aus der nährlösung des pilzes Botrytis cinerea. Chem. Ber. 107, 1720-1730.

Gelderblom, W.C.A., Thiel, P.G., van der Merwe, K.J., Marasas, W.F.O., Spies, H.S.C., 1983. A mutagen produced by Fusarium moniliforme. Toxicon 21, $467-473$.

Gelderblom, W.C.A., Jaskiewicz, K., Marasas, W.F.O., Thiel, P.G., Horak, R.M., Vleggaar, R., Kriek, N.P.J., 1988. Fumonisins-novel mycotoxins with cancer-promoting activity produced by Fusarium moniliforme. Appl. Environ. Microbiol. 54, 1806-1811.

Hanson, J.R., 2008. The chemistry of fungi. The Royal Society of Chemistry. Chapter $1,1-17$.

Jacyno, J.M., Harwood, J.S., Cutler, H.G., Dulik, D.M., 1994. Structure and solution-state conformation of botcinolide, a new biologically active metabolite from the fungus Botrytis cinerea. Int. J. Rapid Publ. Crit. 50, 11585-11592.

Jansen, C., von Wettstein, D., Schäfer, W., Kogel, K.H., Felk, A., Maier, F.J., 2005. Infection patterns in barley and wheat spikes inoculated with wild-type and trichodiene synthase gene disrupted Fusarium graminearum. Proc. Natl. Acad. Sci. 102, 16892-16897.

Jonkers, W., Rodriguez Estrada, A.E., Lee, K., Breakspear, A., May, G., Kistler, H.C., 2012. Metabolome and transcriptome of the interaction between Ustilago maydis and Fusarium verticillioides in vitro. Appl. Environ. Microbiol. 78, 3656-3667. 
Kleigrewe, K., Aydin, F., Hogrefe, K., Piecuch, P., Bergander, K., Würthwein, E.U., Humpf, H.U., 2012. Structure elucidation of new fusarins revealing insights in the rearrangement mechanisms of the Fusarium mycotoxin fusarin C. J. Agric. Food Chem. 60, 5497-5505.

Klein, D.A., Paschke, M.W., 2004. Filamentous fungi: the indeterminate lifestyle and microbial ecology. Microb. Ecol. 47, 224-235.

König, C.C., Scherlach, K., Schroeckh, V., Horn, F., Nietzsche, S., Brakhage, A.A., Hertweck, C., 2013. Bacterium induces cryptic meroterpenoid pathway in the pathogenic fungus Aspergillus fumigatus. Chem. Bio. Chem. 14, 938-942.

Leroch, M., Kretschmer, M., Hahn, M., 2011. Fungicide resistance phenotypes of Botrytis cinerea isolates from commercial vineyards in south west Germany. J. Phytopathol. 159, 63-65.

Li, C., Zhang, J., Shao, C., Ding, W., She, Z., Lin, Y., 2011. A new xanthone derivative from the co-culture broth of two marine fungi (strain No. E33 and K38). Chem. Nat. Compd. 47, 382-384.

Li, G.Q., Huang, H.C., Kokko, E.G., Acharya, S.N., 2002. Ultrastructural study of mycoparasitism of Gliocladium roseum on Botrytis cinerea. Bot. Bull. Acad. Sin. 43, 211-218.

Marasas, W.F.O., Riley, R.T., Hendricks, K.A., Stevens, V.L., Sadler, T.W., Gelineau-van Waes, J., Missmer, S.A., Cabrera, J., Torres, O., Gelderblom, W.C.A., Allegood, J., Martínez, C., Maddox, J., Miller, J.D., Starr, L., Sullards, M.C., Roman, A.V., Voss, K.A., Wang, E., Merrill, A.H., 2004. Fumonisins disrupt sphingolipid metabolism, folate transport, and neural tube development in embryo culture and in vivo: a potential risk factor for human neural tube defects among populations consuming fumonisin-contaminated maize. J. Nutr. 134, 711-716.

Marin, S., Sanchis, V., Vinas, I., Canela, R., Magan, N., 1995. Effect of water activity and temperature on growth and fumonisin B1 and B2 production by Fusarium proliferatum and F. moniliforme on maize grain. Lett. Appl. Microbiol. 21, 298-301. 
Montesinos, E., Bonaterra, A., Badosa, E., Francés, J., Alemany, J., Llorente, I., Moragrega, C., 2002. Plant-microbe interactions and the new biotechnological methods of plant disease control. Int. Microbiol. 5, 169-175.

Müller, M.E.H., Steier, I., Köppen, R., Siegel, D., Proske, M., Korn, U., Koch, M., 2012. Cocultivation of phytopathogenic Fusarium and Alternaria strains affects fungal growth and mycotoxin production. J. Appl. Microbiol. 113, 874-887.

Munkvold, G.P., McGee, D.C., Carlton, W.M., 1997. Importance of different pathways for maize kernel infection by Fusarium moniliforme. Phytopathology 87, 209-217.

Musser, S.M., Gay, M.L., Mazzola, E.P., Plattner, R.D., 1996. Identification of a new series of fumonisins containing 3-hydroxypyridine. J. Nat. Prod. 59, 970-972.

Nelson, P.E., Plattner, R.D., Shackelford, D.D., Desjardins, A.E., 1991. Production of fumonisins by Fusarium moniliforme strains from various substrates and geographic areas. Appl. Environ. Microbiol. 57, 2410-2412.

Nishiuchi, T., Masuda, D., Nakashita, H., Ichimura, K., Shinozaki, K., Yoshida, S., Kimura, M., Yamaguchi, I., Yamaguchi, K., 2006. Fusarium phytotoxin trichothecenes have an elicitor-like activity in Arabidopsis thaliana, but the activity differed significantly among their molecular species. Mol. Plant. Microbe Interact. 19, 512-520.

Noriko, T., Yukako, O., Hiroshi, T., Tatsuo, A., Michio, N., Satoshi, O., 1999. Structure elucidation of roselipins, inhibitors of diacylglycerol acyltransferase produced by Gliocladium roseum KF-1040. J. Antibiotics 52, 815-826.

Nützmann, H.W., Reyes-Dominguez, Y., Scherlach, K., Schroeckh, V., Horn, F., Gacek, A., Schümann, J., Hertweck, C., Strauss, J., Brakhage, A.A., 2011. Bacteria-induced natural product formation in the fungus Aspergillus nidulans requires Saga/Ada-mediated histone acetylation. Proc. Natl. Acad. Sci. 108, $14282-14287$.

Oh, D.C., Kauffman, C.A., Jensen, P.R., Fenical, W., 2007. Induced production of emericellamides A and B from the marine-derived fungus Emericella sp. in 
competing co-culture. J. Nat. Prod. 70, 515-520.

Onaka, H., Mori, Y., Igarashi, Y., Furumai, T., 2011. Mycolic acid-containing bacteria induce natural-product biosynthesis in streptomyces species. Appl. Environ. Microbiol. 77, 400-406.

Otani, H., Kohmoto, K., Kodama, M., 1995. Alternaria toxins and their effects on host plants. Can. J. Bot. 73, 453-458.

Pachenari, A. and Dix, N.J., 1980. Production of toxins and wall degrading enzymes by Gliocladium roseum. Trans. Br. Mycol. Soc. 74, 561-566.

Parada, R.Y., Oka, K., Yamagishi, D., Kodama, M., Otani, H., 2007. Destruxin B produced by Alternaria brassicae does not induce accessibility of host plants to fungal invasion. Physiol. Mol. Plant Pathol. 71, 48-54.

Pedras, M.S.C., Irina, Z.L., Ward, D.E., 2002. The destruxins: synthesis, biosynthesis, biotransformation, and biological activity. Phytochemistry 59, 579-596.

Presello, D.A., Botta, G., Iglesias, J., Eyhérabide, G.H., 2008. Effect of disease severity on yield and grain fumonisin concentration of maize hybrids inoculated with Fusarium verticillioides. Crop Prot. 27, 572-576.

Proctor, R.H., Brown, D.W., Plattner, R.D., Desjardins, A.E., 2003. Co-expression of 15 contiguous genes delineates a fumonisin biosynthetic gene cluster in Gibberella moniliformis. Fungal Genet. Biol. 38, 237-249.

Radwan, O., Li, M., Calla, B., Li, S., Hartman, G.L., Clough, S.J., 2013. Effect of Fusarium virguliforme phytotoxin on soybean gene expression suggests a role in multidimensional defence. Mol. Plant Pathol. 14, 293-307.

Rodriguez Estrada, A.E., Hegeman, A., Kistler, H.C., May, G., 2011. In vitro interactions between Fusarium verticillioides and Ustilago maydis through real-time PCR and metabolic profiling. Fungal Genet. Biol. 48, 874-885.

Ross, P.F., Nelson, P.E., Richard, J.L., Osweiler, G.D., Rice, L.G., Plattner, R.D., Wilson, T.M., 1990. Production of fumonisins by Fusarium moniliforme and Fusarium proliferatum isolates associated with equine leukoencephalomalacia and a pulmonary edema syndrome in swine. Appl. Environ. Microbiol. 56, 3225-3226. 
Sanchez-Rangel, D., SanJuan-Badillo, A., Plasencia, J., 2005. Fumonisin production by Fusarium verticillioides strains isolated from maize in Mexico and development of a polymerase chain reaction to detect potential toxigenic strains in grains. J. Agric. Food Chem. 53, 8565-8571.

Savard, M.E., Miller, J.D., 1992. Characterization of fusarin F, a new fusarin from Fusarium moniliforme. J. Nat. Prod. 55, 64-70.

Schroeckh, V., Scherlach, K., Nützmann, H.W., Shelest, E., Schmidt-Heck, W., Schuemann, J., Martin, K., Hertweck, C., Brakhage, A.A., 2009. Intimate bacterial-fungal interaction triggers biosynthesis of archetypal polyketides in Aspergillus nidulans. Proc. Natl. Acad. Sci. 106, 14558-14563.

Schroers, H., Samuels, G., Seifert, K., Gams, W., 1999. Classification of the mycoparasite Gliocladium roseum in Clonostachys as C. rosea, its relationship to Bionectria ochroleuca, and notes on other Gliocladium-like fungi. Mycologia 91, 365-385.

Shiina, I., Fukui, H., 2009. Chemistry and structural determination of botcinolides, botcinins and botcinic acids. Chem. Commun. 385-400.

Sondergaard, T.E., Hansen, F.T., Purup, S., Nielsen, A.K., Bonefeld-Jørgensen, E.C., Giese, H., Sørensen, J.L., 2011. Fusarin C acts like an estrogenic agonist and stimulates breast cancer cells in vitro. Toxicol. Lett. 205, 116-121.

Sterner, O., Thines, E., Eilbert, F., Anke, H., 1998. Glisoprenins C, D and E, new inhibitors of appressorium formation in Magnaporthe grisea, from cultures of Gliocladium roseum 2. Structure determination. J. Antibiotics 51, 228-231.

Strange, R.N., Scott, P.R., 2005. Plant disease: a threat to global food security. Annu. Rev. Phytopathol. 43, 83-116.

Strobel, G.A., Knighton, B., Kluck, K., Ren, Y., Livinghouse, T., Griffin, M., Spakowicz, D., Sears, J., 2008. The production of myco-diesel hydrocarbons and their derivatives by the endophytic fungus Gliocladium roseum (NRRL 50072). Microbiology 154, 3319-3328.

Sutton, J.C., Li, D.W., Peng, G., Yu, H., Zhang, P., Valdebenito-Sanhueza, R.M., 1997. Gliocladium roseum a versatile adversary of Botrytis cinerea in crops. Plant 
Dis. $81,316-328$.

Takaoka, S., Kurata, M., Harimoto, Y., Hatta, R., Yamamoto, M., Akimitsu, K. and Tsuge, T., 2014. Complex regulation of secondary metabolism controlling pathogenicity in the phytopathogenic fungus Alternaria alternata. New Phytologist 202, 1297-1309.

Tani, H., Koshino, H., Sakuno, E., Nakajima, H., 2005. Botcinins A, B, C, and D, metabolites produced by Botrytis cinerea, and their antifungal activity against Magnaporthe grisea, a pathogen of rice blast disease. J. Nat. Prod. 68, 17681772.

Tani, H., Koshino, H., Sakuno, E., Cutler, H.G., Nakajima, H., 2006. Botcinins E and $\mathrm{F}$ and botcinolide from Botrytis cinerea and structural revision of botcinolides. J. Nat. Prod. 69, 722-725.

Thines, E., Eilbert, F., Sterner, O., Anke, H., 1997. Glisoprenin A, an inhibitor of the signal transduction pathway leading to appressorium formation in germinating conidia of Magnaporthe grisea on hydrophobic surfaces. FEMS Microbiol. Lett. 151, 219-224.

UIPP, 2002. Union des Industries de la Protection des Plantes. Annu. Rep. Paris, France.

Utermark, J., Karlovsky, P., 2007. Role of Zearalenone lactonase in protection of Gliocladium roseum from fungitoxic effects of the mycotoxin zearalenone. Appl. Environ. Microbiol. 73, 637-642.

Velluti, A., Marin, S., Gonzalez, R., Ramos, A., Sanchis, V., 2001. Fumonisin B1, zearalenone and deoxynivalenol production by Fusarium moniliforme, $F$. proliferatum and F. graminearum in mixed cultures on irradiated maize kernels. J. Sci. Food Agric. 81, 88-94.

Wang, E., Norred, W.P., Bacon, C.W., Riley, R.T., Merrill, A.H., 1991. Inhibition of sphingolipid biosynthesis by fumonisins. Implications for diseases associated with Fusarium moniliforme. J. Biol. Chem. 266, 14486-14490.

Wang, J., Lin, W., Wray, V., Lai, D., Proksch, P., 2013. Induced production of depsipeptides by co-culturing Fusarium tricinctum and Fusarium begoniae. 
Tetrahedron Lett. 54, 2492-2496.

Washington, W.S., 1993. Recent advances in Botrytis research. Australas. Plant Pathol. $22,156-157$.

Welmar, K., Tschesche, R., Breitmaier, E., 1979. Botrylacton, ein neuer wirkstoff aus der nährlösung des Pilzes Botrytis cinerea. Chem. Ber. 112, 3598-3602.

Xue, A.G., 1999. Gliocladium roseum strains useful for the control of fungal pathogens in plants. WO 2000018241 A1 20000406.

Yabuta, T., Kambe, K., Hayashi, T., 1934. Biochemistry of the Bakanae-fungus, I. fusarinic acid, a new product of the Bakanae-fungus. J. Agric. Chem. Soc. 10, $1059-1068$.

Yu, H. and Sutton, J.C., 1997. Morphological development and interactions of Gliocladium roseum and Botrytis cinerea in raspberry. Can. J. Plan Pathol. 19, 237-246.

Zhang, L., Yang, J., Niu, Q., Zhao, X., Ye, F., Liang, L., Zhang, K.Q., 2008. Investigation on the infection mechanism of the fungus Clonostachys rosea against nematodes using the green fluorescent protein. Appl. Microbiol. Biotechnol. 78, 983-990.

Zuck, K.M., Shipley, S. and Newman, D.J., 2011. Induced production of N-formyl alkaloids from Aspergillus fumigatus by co-culture with Streptomyces peucetius. J. Nat. Prod. 74, 1653-1657. 
Chapter 2: Interactions among the fungi Aspergillus niger, Fusarium verticillioides, and Gliocladium roseum: effects on fungal biomass, metabolic diversity and fumonisin production

\author{
Subhankar Chatterjee, Yi Kuang, Richard Splivallo, Paramita Chatterjee, \\ Petr Karlovsky*
}

\title{
Author Contributions
}

YK carried out the experiments of fumonisin analysis in single and dual culture of F. verticillioides with G. roseum and wrote the part of this manuscript.

\begin{abstract}
Background

Interactions among fungi colonizing dead organic matter are dominated by exploitation competition and interference competition. The major mechanism of interference competition is thought to be antibiosis caused by secondary metabolites. However, the effect of competition on secondary metabolite production by fungi is poorly understood.
\end{abstract}

\section{Methods and principal findings}

The saprophyte Aspergillus niger, the phytopathogen Fusarium verticillioides, and the mycoparasite Gliocladium roseum were grown in single and dual culture. Fungal biomass was determined based on restriction fragments of co-amplified 28S RNA 
genes. Secreted metabolites were analyzed by HPLC-MS, and signal intensities were normalized according to producer biomass. Most metabolites produced early disappeared over time. Dual cultures accumulated numerous new metabolites not produced in single cultures. Many metabolites occurring in single cultures were suppressed in dual cultures, few were stimulated or degraded. Production of the mycotoxin fumonisin $\mathrm{B}$ by $F$. verticillioides was reduced in the co-cultures with G. roseum.

\section{Conclusions and significance}

The ability to take-up and catabolize self-secreted metabolites may be widespread among fungi. Fungal interactions affect the diversity of secreted metabolites primarily by suppressing the synthesis of metabolites produced in single culture and by inducing the synthesis of new metabolites. The latter substantially increases the metabolic diversity in mixed cultures. The production of fumonisin B by F. verticillioides was reduced by co-cultivating with the mycoparasite G. roseum, indicating that $G$. roseum may reduce the mycotoxin levels in the commodity against F. verticillioides.

Keywords: Fungal competition, metabolic diversity, interference competition, fumonisin, metabolic profiling

\section{Introduction}

Dead organic matter is rapidly colonized by a complex community of microorganisms that includes saprophytic fungi. Obligate saprophytic fungi feed on dead organic matter during their entire life, while many phytopathogenic and entomopathogenic fungi depend on saprophytic growth when they form propagules at the end of their life cycle. Trichoderma, Gliocladium, and other mycoparasitic fungi with a wide range of hosts feed either on living fungal mycelia or on dead organic matter and may also colonize living plants. The interactions among saprophytic fungi are dominated by 
competition.

Interspecific competition is formally divided into exploitation competition, which occurs when the use of a resource by one species reduces its availability for the another species, and interference competition, which occurs when one species directly restrains the growth or spread of a competitor. In microbial communities, exploitation competition reduces the access of a competitor to a substrate by its depletion while interference competition inhibits competitor growth by antibiosis (Wicklow, 1981).

Filamentous fungi secrete mixtures of secondary metabolites, many of which possess prominent biological activities (Keller et al., 2005). Although specific biological functions have been demonstrated for very few of them, these secreted substances contribute to the fitness of their producers at some stage of their life cycle or under specific, repeatedly occurring conditions. The ecological metabolite hypothesis defines ecological metabolites as compounds affecting the interactions of their producers with other organisms (Sirenko et al., 1979). The current view of fungal ecological chemistry is that most, if not all, secreted fungal secondary metabolites are ecological metabolites (Karlovsky, 2008). The best-established function of ecological metabolites is antibiotic activity that inhibits competing microorganisms (O'Brien and Wright, 2011). Any physiological or developmental process may be the target of secondary metabolites. For instance, ecological metabolites can inhibit hydrolytic enzyme activity (Omura et al., 2000) or synthesis (Lutz et al., 2003), and can inhibit secondary metabolite synthesis (Child et al., 1998; Cooney at al., 2001). In response to antibiotic metabolites produced by a competitor, a fungus may detoxify the metabolite (Cooney et al., 2001; Utermark and Karlovsky, 2007) or may synthesize and secrete its own toxic metabolites (Losada et al., 2009).

Genomes of filamentous fungi harbor numerous gene clusters putatively involved in the synthesis of secondary metabolites. Many of these clusters appear to be silent; the corresponding metabolic products are mostly unknown (Keller et al., 2005). It only recently became apparent that the expression of many of these gene clusters is induced by biotic interactions (Oh et al., 2007; Scherlach and Hertweck, 2009; Brakhage and Schroeckh, 2011; Schroeckh et al., 2009). Reoccurring interactions 
drive the selection for antibiotic production, as recently demonstrated by competition-based laboratory evolution of a Streptomyces spp. (Charusanti et al., 2012). The genetic repertoire for secondary metabolites production and its control thus reflects the adaptation of fungi to biotic interactions.

Several studies have considered the effects of fungal interactions on mycotoxin production. Interaction with other fungi often inhibited but only infrequently stimulated mycotoxin synthesis (Velluti et al., 2000; Marin et al., 2001; Ramakrishna et al., 1996). Rodriguez Estrada et al. (2011) recently compared the growth and metabolic profiles of F. verticillioides and Ustilago maydis interacting on agar plates. The authors reported that Fusarium suppressed the growth of Ustillago and induced the production of Ustillago metabolites, which were hypothesized to function as antibiotics against Fusarium. Surprisingly, Fusarium biomass was greater when growing in dual culture with Ustillago than when growing alone. The authors suggested that Fusarium might have acted as a mycoparasite, i.e., it might have killed and then consumed Ustillago cells.

In this work, we use metabolic profiling to investigate the induction, inhibition and degradation of secreted fungal metabolites in dual cultures of fungi. A common saprophyte (Aspergillus niger, A.n.), a phytopathogen (Fusarium verticillioides, F.v.), and a mycoparasite with a saprophytic life phase (Gliocladium roseum, G.r.) were selected as interacting partners. The use of all dual combinations of the three species allowed us to determine whether competitive fitness is "congruent" (i.e., whether relative fitness of strains $\mathrm{A}, \mathrm{B}$ and $\mathrm{C}$ following the patterns $\mathrm{A}>\mathrm{B}$ and $\mathrm{B}>\mathrm{C}$ implies $\mathrm{A}$ $>$ C) or "incongruent" (i.e., whether relative fitness of strains A, B and C may follow the patterns $\mathrm{A}>\mathrm{B}, \mathrm{B}>\mathrm{C}$, and $\mathrm{C}>\mathrm{A}$ ). Furthermore, we determined: the extent to which metabolites secreted in single cultures are induced or suppressed in dual cultures; the extent to which new metabolites are synthesized in dual cultures; whether such effects are species specific; and how such effects relate to changes in fungal biomass over time. 


\section{Materials and methods}

\section{Fungi and media}

The fungi studied included the saprophyte Aspergillus niger 14.203 (culture collection of the Division of Phytopathology, University of Göttingen, Germany, provided by A. von Tiedemann), the mycoparasite Gliocladium roseum DSM 62726 (syn. Clonostachys rosea; obtained from Deutsche Sammlung von Mikroorganismen und Zellkulturen, Braunschweig, Germany), and the phytopathogen Fusarium verticillioides FRC M-8114 (obtained from the Fusarium Research Center, PA, USA). The fungi were maintained on potato dextrose agar plates. Potato dextrose agar was purchased from Carl Roth GmbH (Karlsruhe, Germany) and prepared according to the manufacturer's instruction. For the experiments, the fungi were grown on agar plates containing GM7 medium (Utermark and Karlovsky, 2007), and spore suspensions were made in sterile-distilled water. For liquid cultures, GM7 medium was prepared in the same way except that glucose was autoclaved separately and no agar for solidification was used.

\section{Chemicals}

All chemicals used were of "pro analysis" quality. Methanol (gradient quality; Fisher Scientific, Schwerte, Germany), acetonitrile (gradient quality; VWR, Darmstadt, Germany), and acetic acid (LCMS grade; Fluka/Sigma-Aldrich, St. Louis, USA) were used for the mobile phases in HPLC. TE buffer contained $10 \mathrm{mM}$ Tris and $1 \mathrm{mM}$ EDTA; the pH was adjusted to 8.0. Analytical standards of fumonisins B1, B2 and B3 were purchased from Biopure (Tulln, Austria).

\section{Culture conditions}

Four replicates of single and dual cultures of Aspergillus niger (A.n.), Fusarium verticillioides (F.v.), and Gliocladium roseum (G.r.) were grown in the dark at $21^{\circ} \mathrm{C}$ in 100-ml Erlenmeyer flasks containing $30 \mathrm{ml}$ of GM7 medium inoculated with $100 \mu \mathrm{L}$ 
of a spore suspension that contained $10^{5}$ spores/mL. For dual cultures (designated F.v./G.r., F.v./A.n., and A.n./G.r.), an equal number of spores from two species was inoculated. Cultures were grown without agitation to prevent the development of mycelia lumps, and the medium in the flask formed only a thin layer $(13 \mathrm{~mm})$, which insured its saturation with oxygen. Fungal mycelium from single and dual cultures was harvested by filtration after 10, 20, and 30 days. The harvested mycelium was freeze-dried. For the fumonisin biotransformation experiment, G.r. with $1 \mathrm{~mL}$ fumonisin $\mathrm{B} 1$ solution $\left(5 \mathrm{mg} \cdot \mathrm{L}^{-1}\right)$ incubated at $21^{\circ} \mathrm{C}$ using the same culture medium as described previously. Four flasks for each treatment were used as replicates. Samples for fumonisin analysis were not extracted or defatted by organic solvent to avoid losses of the mycotoxins in the organic phase.

\section{Determination of fungal biomass in dual cultures by densitometry of species-specific fragments of $28 \mathrm{~S}$ genes}

Principle. Total biomass of both fungi in a dual culture was determined as the weight of the freeze-dried mycelium. Densitometry of species-specific restriction fragments of 28S RNA genes was used to estimate the proportion of each species in the total biomass in the mixed culture. For this purpose, a 900-bp fragment of the 28S RNA gene was amplified using primers common to all three fungal species in the study and was digested by restriction enzymes to produce species-specific fragments. The relative intensities of the fragments, corrected for fragment length, were used to calculate the absolute biomass of each species; we assumed that the copy number of the amplified sequence was identical for all three species. The lengths of the restriction fragments of the amplified portion of the 28S RNA gene for A.n., F.v., and G.r. used in this study are listed in Table 1. Fungal biomass in single cultures was determined as the dry weight of the mycelium. 
Table 1. Restriction enzymes used and DNA fragment size

\begin{tabular}{|l|c|c|c|}
\hline \multirow{2}{*}{$\begin{array}{l}\text { Species } \\
\text { combination } \\
\text { (restriction } \\
\text { enzymes) }\end{array}$} & $\begin{array}{c}|c| \\
\text { Aspergillus niger } \\
\text { (A.n.) }\end{array}$ & $\begin{array}{c}\text { Fusarium verticillioides } \\
(\text { F.v. })\end{array}$ & $\begin{array}{c}\text { Gliocladium roseum } \\
\text { (G.r.) }\end{array}$ \\
\hline A.n./F.v. (MseI) & $617 *+307$ & $550^{*}+300+57$ & - \\
\hline $\begin{array}{l}\text { A.n./G.r. } \\
\text { (MseI+ApoI) }\end{array}$ & $\begin{array}{l}500^{*}+193+117+ \\
87+27\end{array}$ & - & $603 *+271+27$ \\
\hline F.v./G.r. (MseI) & - & $550^{*}+300+57$ & $603^{*}+298$ \\
\hline
\end{tabular}

*Fragments used for quantification.

Genomic DNA isolation from single and dual cultures. Lyophilized mycelium was ground in a ball mill (Mixer Mill MM 200, Retsch, Hann, Germany) in a 2-mL tube with five wolfram carbide spheres (diameter $3 \mathrm{~mm}$ ) for $30 \mathrm{~s}$ at maximum speed. For DNA extraction, a variant of the CTAB method was used as described earlier (Brandfass and Karlovsky, 2006). The quality and quantity of DNA was assessed by electrophoresis in $0.8 \%(\mathrm{w} / \mathrm{v})$ agarose gels (Cambrex, Rockland, ME, USA) prepared in TAE buffer (40 mM Tris, $1 \mathrm{mM}$ EDTA, $\mathrm{pH}$ adjusted to 8.5 with acetic acid). The electrophoresis was carried out at $4 \mathrm{~V} \cdot \mathrm{cm}^{-1}$ for $60 \mathrm{~min}$. The gels were stained with ethidium bromide $\left(2 \mathrm{mg} \cdot \mathrm{L}^{-1}\right)$ and documented after irradiation with UV light at 360 nm with a digital imaging system (Vilber Lourmat, Marne la Vallee, France). The densitometry was performed using Multi Analyst-Software (BioRad, Hercules, CA, USA). The concentration of fungal DNA was calculated by comparing a dilution series with defined amounts of DNA of lambda phage (methylated, from Escherichia coli host strain W3110).

PCR amplification and restriction digestion. PCR amplification was carried out in a $25-\mu \mathrm{L}$ reaction mixture containing $1 \mathrm{X}$ PCR buffer (from 10X reaction buffer: 670 $\mathrm{mM}$ Tris- $\mathrm{HCl}, 160 \mathrm{mM}\left(\mathrm{NH}_{4}\right)_{2} \mathrm{SO}_{4}, 0.1 \%$ (v/v) Tween-20, $\mathrm{pH} 8.8$ at $25^{\circ} \mathrm{C}$; Bioline, Luckenwalde, Germany), $3 \mathrm{mM} \mathrm{MgCl}_{2}, 0.2 \mathrm{mM}$ of each deoxyribonucleotide triphosphate (Bioline, Luckenwalde, Germany), $0.5 \mu \mathrm{M}$ of each primer (forward: 
CTAATCATTCGCTTTACCTCATAAAACTGA), 0.4 units of Taq DNA polymerase (BIOTaq, Bioline, Luckenwalde, Germany), and $2 \mu \mathrm{L}$ of template DNA.

The TPersonal thermocycler (Biometra, Göttingen, Germany) was used for PCR amplification. The condition were: an initial denaturation for 2 min at $94^{\circ} \mathrm{C}$; followed by 35 cycles of $30 \mathrm{~s}$ denaturation at $94^{\circ} \mathrm{C}, 30 \mathrm{~s}$ annealing at $61^{\circ} \mathrm{C}$, and $60 \mathrm{~s}$ elongation at $72^{\circ} \mathrm{C}$; and a final extension for $5 \mathrm{~min}$ at $72^{\circ} \mathrm{C}$. The amplification was checked by agarose electrophoresis as described above. PCR products were precipitated with ethanol and dissolved in $25 \mu \mathrm{L}$ of sterile TE buffer.

A $10-\mu \mathrm{L}$ volume of purified PCR products was either digested with $5 \mathrm{u}$ of MseI (for DNA of F.v./G.r. and F.v./A.n.) or was doubly digested with $2.5 \mathrm{u}$ each of MseI and ApoI (for DNA of A.n./G.r.) in a $25-\mu \mathrm{L}$ reaction mixture following the procedure described by the manufacturer of the enzymes (Fermentas, Germany). To obtain DNA bands that could be quantified by densitometry, different dilutions of the digestion products were loaded on agarose gels. For A.n./G.r., 1.7\% (w/v) agarose gel was used, and the separation was carried out at $4 \mathrm{~V} \mathrm{~cm}^{-1}$ for $60 \mathrm{~min}$. For F.v./G.r. and F.v./A.n., $3 \%$ (w/v) low-MW agarose (Biozyme Scientific, Oldendorf, Germany) was used, and the separation was carried out at $4 \mathrm{~V} \mathrm{~cm}^{-1}$ for $210 \mathrm{~min}$. Gels were stained with ethidium bromide $\left(2 \mathrm{mg} \cdot \mathrm{L}^{-1}\right)$ and visualized and photographed in UV light using a 128-bit camera (Vilber Lourmat, Eberhardzell, Germany).

Densitometric analysis and biomass estimation. Intensities of DNA bands were determined using Quantity One software version 4.5 (BioRad, Hercules, USA). Relative intensities were normalized by the size of DNA fragments (Table 1) and multiplied by the dry weight of mycelium obtained from dual cultures to determine the biomass of each species.

\section{Estimation of the effect of competition on fitness}

To compare the effect of interaction between three fungi on their biomass in all dual combinations, we calculated the relative biomass of strain $i$ to strain $j$ in single cultures and dual cultures as follows: 


$$
\begin{aligned}
& \mathrm{rB}_{\mathrm{i}(\mathrm{s}, \mathrm{j})}=2 * \mathrm{~B}_{\mathrm{i}(\mathrm{s})} /\left[\mathrm{B}_{\mathrm{i}(\mathrm{s})}+\mathrm{B}_{\mathrm{j}(\mathrm{s})}\right] \\
& \mathrm{rB}_{\mathrm{i}(\mathrm{d}, \mathrm{j})}=2 * \mathrm{~B}_{\mathrm{i}(\mathrm{d}, \mathrm{j})} /\left[\mathrm{B}_{\mathrm{i}(\mathrm{d}, \mathrm{j})}+\mathrm{B}_{\mathrm{j}(\mathrm{d}, \mathrm{i})}\right]
\end{aligned}
$$

where:

- $\mathrm{rB}_{\mathrm{i}(\mathrm{s}, \mathrm{j})}$ is the relative biomass of strain i compared to strain $\mathrm{j}$ in single culture,

$\circ \mathrm{B}_{\mathrm{i}(\mathrm{s})}$ is the biomass of strain $\mathrm{i}$ in single culture,

$\circ B_{j(s)}$ is the biomass of strain $\mathrm{j}$ in single culture,

○ $\mathrm{rB}_{\mathrm{i}(\mathrm{d}, \mathrm{j})}$ is the relative biomass of strain $\mathrm{i}$ in dual culture with strain $\mathrm{j}$,

○ $B_{i(d, j)}$ is the biomass of strain $i$ in dual culture with strain $j$, and

- $B_{j(d, i)}$ is the biomass of strain $\mathrm{j}$ in dual culture with strain $\mathrm{i}$.

The effect of the interaction with strain $\mathrm{j}$ on biomass of strain $\mathrm{i}$ was determined as the ratio of relative biomass in dual and single cultures:

$$
\mathrm{rF}_{\mathrm{i}(\mathrm{j})}=\left[\mathrm{rB}_{\mathrm{i}(\mathrm{d}, \mathrm{j})} / \mathrm{rB}_{\mathrm{i}(\mathrm{s}, \mathrm{j})}\right]-1
$$

$\mathrm{rF}_{\mathrm{i}(\mathrm{j})}$ was designated the index of competitive fitness. In the absence of competition, the biomass of both fungi in a dual culture is expected to grow at the same rate as in single cultures:

$$
\mathrm{rF}_{\mathrm{i}(\mathrm{j})}=\mathrm{rF}_{\mathrm{j}(\mathrm{i})}=0
$$

If interactions affect growth, $\mathrm{rF}_{\mathrm{i}(\mathrm{j})}$ and $\mathrm{rF}_{\mathrm{j}(\mathrm{i})}$ will diverge from 0 in opposite directions:

$$
\left(\left(\mathrm{rF}_{\mathrm{i}(\mathrm{j})}<0\right) \text { AND }\left(\mathrm{rF}_{\mathrm{j}(\mathrm{i})}>0\right)\right) \text { OR }\left(\left(\mathrm{rF}_{\mathrm{i}(\mathrm{j})}>0\right) \text { AND }\left(\mathrm{rF}_{\mathrm{j}(\mathrm{i})}<0\right)\right)
$$

A positive value of $\mathrm{rF}_{\mathrm{i}(\mathrm{j})}$ indicates that strain $\mathrm{i}$ gains advantage over strain $\mathrm{j}$ or suffers less inhibition than strain $\mathrm{j}$ in dual cultures of $\mathrm{i}$ and $\mathrm{j}$.

\section{Metabolic profiling of secreted fungal metabolites by HPLC-ESI-MS}

Extraction of secreted metabolites. Single and dual cultures harvested at three time points were filtered, and supernatants were extracted twice with an equal volume of n-hexane for defatting. A $25-\mathrm{mL}$ volume of each defatted supernatant was extracted three times with an equal volume of ethyl acetate (EtOAc). Combined extracts were evaporated to dryness under vacuum at $30{ }^{\circ} \mathrm{C}$. The residue was dissolved in $1 \mathrm{~mL}$ of 
methanol/bi-distilled water $(1: 1)$, and the solution was filtered through a $0.2-\mu \mathrm{m}$ Teflon filter (WICOM, Heppenheim, Germany). The solution was immediately subjected to HPLC-MS analysis or was stored at $-20{ }^{\circ} \mathrm{C}$.

Metabolic profiling by HPLC-MS. For non-targeted metabolite analysis, a reverse-phase HPLC system coupled to an electrospray and ion trap detector 500-MS (Varian) was used as described before (Ratzinger et al., 2009). The mobile phase consisted of a binary gradient of $7 \mathrm{mM}$ acetic acid in $95 \%$ water $/ 5 \%$ acetonitrile (A) and $7 \mathrm{mM}$ acetic acid in methanol (B): 0-5 min 90\% A; 5-30 min from 90\% A to $2 \%$ $\mathrm{A} ; 30-38 \mathrm{~min} 2 \% \mathrm{~A}$; and $38-40 \min 2 \%$ A to $90 \% \mathrm{~A}$ at a flow rate of $0.2 \mathrm{~mL} / \mathrm{min}$. This was followed by washing and re-equilibration steps. A quality control sample was injected at the beginning, in the middle, and at the end of each sequence to monitor the stability of the method.

Ionization was done by electrospray both in positive and negative mode with the following parameters (negative/positive): needle voltage $-4,500 \mathrm{~V} /+5,000 \mathrm{~V}$, shield voltage $-600 \mathrm{~V} /+600 \mathrm{~V}$, capillary voltage $-/+40 \mathrm{~V}$, drying gas (nitrogen) 25 psi (172.5 $\mathrm{kPa}$ ) at $250{ }^{\circ} \mathrm{C}$, and nebulizing gas (air) $50 \mathrm{psi}(345 \mathrm{kPa}$ ). The MS analyzer was operated in full-scan mode, mass range $m / z$ 100-1000, scan speed $5000 \mathrm{Da} / \mathrm{s}$, and three scans averaged. For data acquisition, MS workstation/MS Data Review 6.9 (Varian) was used. Because mass spectrometry signals after ESI originate from molecular ions, the terms MS signals and metabolites are used synonymously in the following text.

Data processing. Raw HPLC-MS data were processed with the Component Detection Algorithm (CODA, Windig et. al., 1996) implemented in ACD/MS Manager v. 8.0 (Advanced Chemistry Development, Toronto, Canada). The CODA algorithm evaluates the quality of chromatographic peaks by calculating a mass quality index (MCQ) that reflects the similarity between the original mass chromatograms and their smoothed and mean-subtracted versions. Data processing by CODA included smoothing, baseline correction, and peak picking. Peak tables contained the monoisotopic mass (mass to charge ratio for $[\mathrm{M}-\mathrm{H}]^{-}$and $[\mathrm{M}+\mathrm{H}]^{+}$), retention time (Rt), 
peak area, and MCQ value for each signal that passed the MCQ thresholds of 0.8 and the $\mathrm{S} / \mathrm{N}$ threshold of 10. After peak alignment, signals occurring in controls (EtOAc extracts of uninoculated medium) and signals detected in fewer than three of four replicates were discarded. Normalization of peak areas for single cultures was accomplished by dividing peak areas by fungal biomass. For dual cultures, peak areas were divided by the biomass of the producer of the pertinent metabolite, which was identified by comparison with metabolic profiles of single species. Signals of unknown origin, which occurred only in dual cultures and never in single cultures, were normalized by dividing peak areas with the average biomass of both fungi. After normalization, corresponding signals in single and dual cultures were compared. Suppressed metabolites were defined as metabolites whose normalized signal intensities were at least 10-times lower in dual cultures than in single cultures. Induced metabolites were defined as metabolites whose signal intensities were at least 10-times larger in dual cultures than in single cultures. Interaction-specific metabolites were defined as signals found only in dual cultures.

\section{Determination of the content of fumonisins}

Two $\mathrm{mL}$ of fermentation broth of each sample was filtered through a $0.8-\mu \mathrm{m}$-pore-size filter. The analysis of fumonisin was performed by HPLC-MS/MS (500 MS, Varian, Germany) as described before (Adejumo et al., 2007). Ten $\mu \mathrm{L}$ sample was injected for the determination of FB1, FB2 and FB3. The separation was carried out using a reverse phase column Kinetex C18 (50.0 mm x 2.1mm, particle size $2.6 \mu \mathrm{m}$, Phenomenex, Germany) at $40^{\circ} \mathrm{C}$ with binary gradient elution. The mobile phase consisted of water with $5 \%$ acetonitrile (A) and methabol (B) both containing $7 \mathrm{mM}$ of acetic acid. Start 30 s for $20 \%$ B, 10 min gradient from $20 \%$ to $98 \%$ B, 4 min kept at $98 \%$ B , 1 min linear gradient from $98 \%$ to $20 \%$ B and 5 min held at $20 \%$ B at a flow rate of $0.2 \mathrm{~mL} / \mathrm{min}$. The protonated ion $\mathrm{m} / \mathrm{z}, 722.5,706$ and 706 in positive ionization mode were used as precursor ion and $\mathrm{m} / \mathrm{z}$ 686.4, 336.6 and 688.4 were selected as product ions for FB1, FB2 and FB3 respectively. The concentration of fumonisin was 
determined by using standard calibration curves prepared with 10 concentrations up to $2.5 \mathrm{mg} \cdot \mathrm{L}^{-1}$. Samples exceeding the concentration of the highest standard were diluted 10-times.

\section{Qualitative data analysis and descriptive statistics}

The HPLC-MS profiles of all samples in form of peak tables were combined in a single matrix containing retention times, $\mathrm{m} / \mathrm{z}$ values, and peak areas. The matrix was transformed into a binary matrix showing the presence or absence of signals. The matrix was used to study the effect of fungal interactions and cultivation time on the diversity of secreted metabolites and to group samples by cluster analysis (UPGMA clustering using Jaccard's similarity indices).

\section{Results}

\section{Fungal biomass in dual cultures}

A.n., F.v. and G.r. were grown for 10, 20, and 30 days in single and dual cultures without agitation. Mycelia were harvested, dried, and weighed. The contribution of each species to the biomass in dual cultures was determined by DNA analysis. The development of fungal biomass over time is shown in Figure 1. G.r. produced the largest biomass at all time points in single cultures, whereas A.n. dominated over F.v. and G.r. in dual cultures (Figure 1). The effects of competition on the fitness of interacting fungi, estimated by comparing their biomass in single and dual cultures, are shown in Table 2. In spite of the dominance of A.n. over F.v. in co-culture, the growth suppression in A.n. in co-cultures with F.v. as compared to single cultures of A.n. was similar (10 days) or even larger (30 days) than the growth suppression experienced by F.v. in co-culture with A.n., as indicated by the indices of competitive fitness (Table 2). 

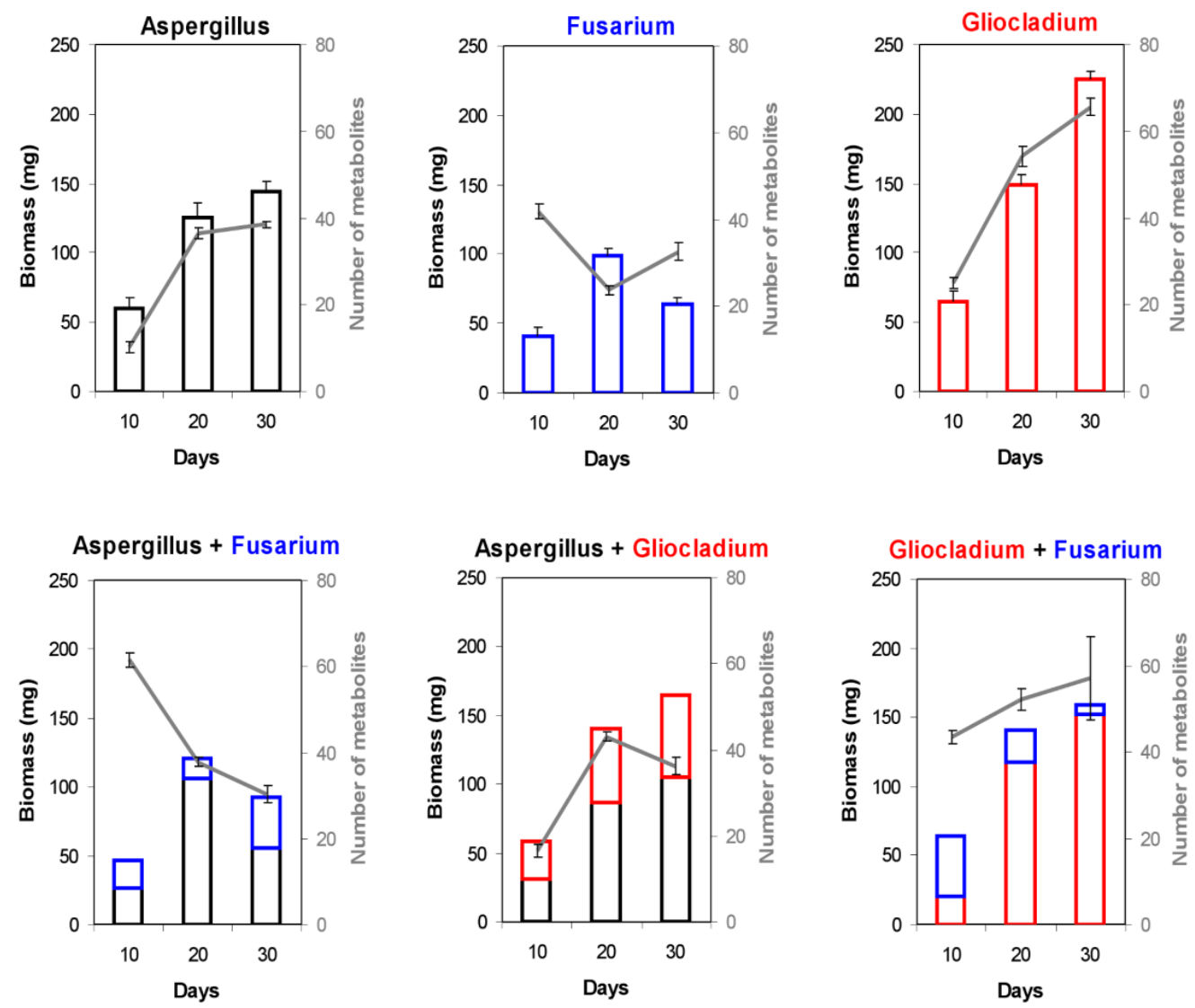

Figure 1. Development of fungal biomass and the number of detected metabolites over time in single and dual cultures. Number of metabolic signals detected by HPLC-MS (grey lines) and the fungal biomass (bars) for single and dual cultures of Aspergillus niger, Fusarium verticillioides, and Gliocladium roseum after 10, 20, and 30 days of incubation are shown. Aspergillus: Aspergillus niger; Fusarium: Fusarium verticillioides; Gliocladium: Gliocladium roseum. Error bars indicated the standard error for the biomass in single cultures and for the number of metabolic signals.

\section{Diversity of secreted metabolites}

The number of metabolites detected in single and dual cultures over time is shown in Figure 1. The total number of metabolites extracted from the supernatants of single cultures of A.n. and G.r. increased over time in parallel with the increase in biomass. This trend was not apparent in F.v. cultures, probably because the mycelium underwent autolysis between day 20 and 30. In dual cultures of A.n. / F.v., the number of detectable metabolites decreased by $50 \%$ during the cultivation period, indicating 
that metabolites secreted at an early stage were taken up and possibly catabolized by aging mycelia. This trend was not apparent for the number of metabolites detected in the supernatants of dual cultures of A.n. / G.r. and G.r. / F.v..

Similarities among aligned metabolic profiles for all cultures were investigated by hierarchical clustering. UPGMA based on Jaccard's similarity indices revealed that the profiles split into two major clusters (Figure 2). Cluster I consisted of metabolites produced by single cultures of F.v. and G.r. and their dual cultures at all time points. Cluster II consisted of A.n. alone and in dual culture with F.v. and G.r. at all time points. The results show that metabolic profiles were less affected by culture age than by interacting species. Cluster II also illustrates the dominance of A.n. metabolites in dual cultures with G.r. and F.v. Subclusters are also evident in Figure 2. After 20 and 30 days, the metabolic profiles of F.v. / G.r. dual cultures clustered separately from those of single cultures of F.v. or G.r., indicating that the metabolic profiles of the dual cultures were dominated by newly formed "interaction-specific" metabolites (defined further in the next section). A similar pattern was evident in the interaction between A.n. and F.v. in that the metabolic profiles of F.v. /A.n. dual cultures formed a subcluster distinct from those of A.n. and F.v. single cultures at all time points. This was, however, not true for the interaction between A.n. and G.r., which was dominated by A.n.. Taken together, these results indicate strong effects of fungal interactions on the composition and complexity of secreted metabolites. These effects are further dissected in the following sections. 


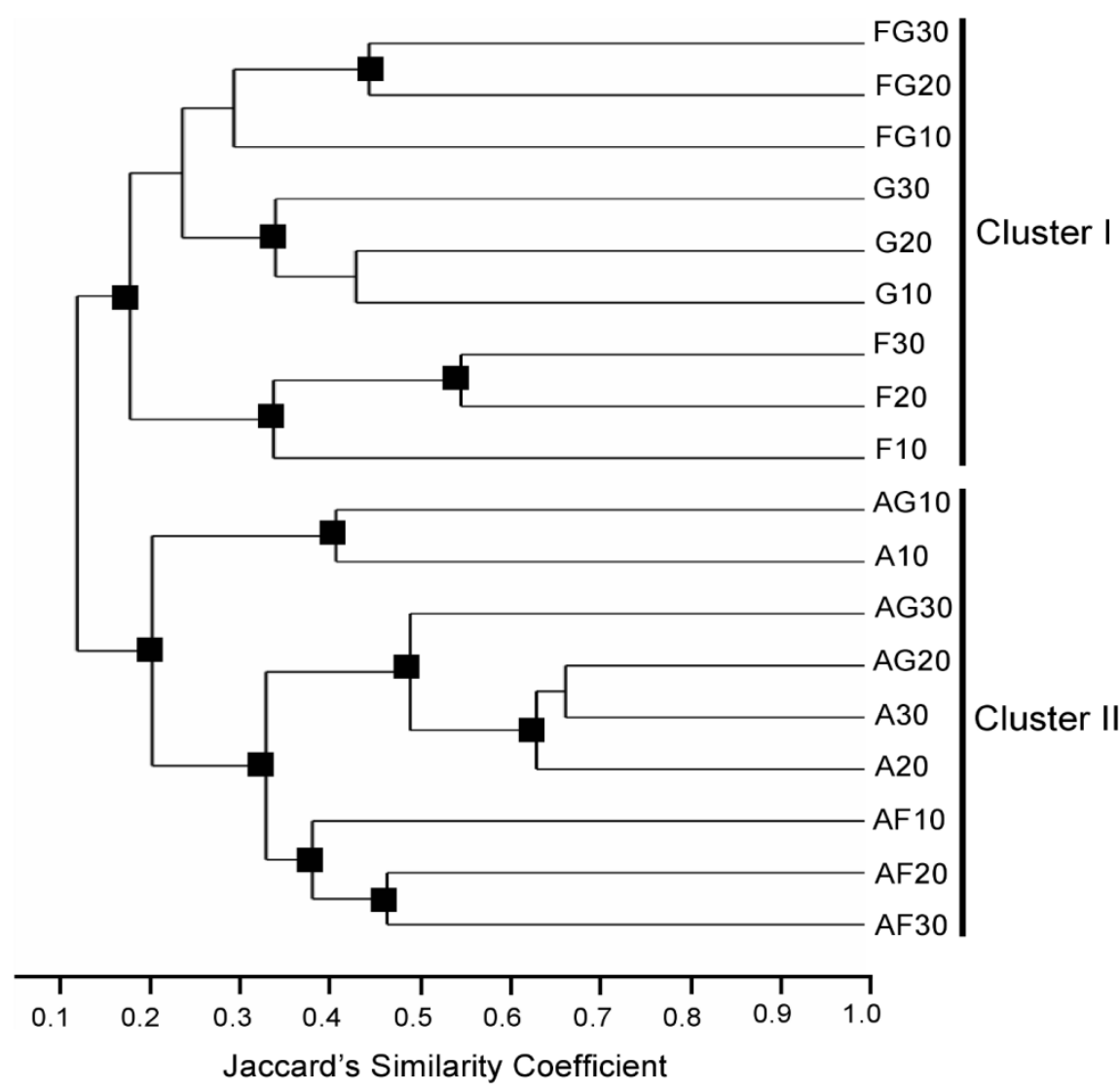

Figure 2. Hierarchical clustering analysis (HCA) based on Jaccard's similarity coefficients $($ coph. coeff. $=0.906)$. Nodes denoted as a were supported by $>75 \%$ bootstraps for 2000 replicates. A: Aspergillus niger; F: Fusarium verticillioides; G: Gliocladium roseum; AG: Aspergillus niger + Gliocladium roseum; FG: Fusarium verticillioides + Gliocladium roseum; AF: Aspergillus niger + Fusarium verticillioides; 10: 10 days, 20: 20 days, 30: 30 days.

\section{Suppression and stimulation of metabolite production by fungal interactions}

We normalized signal intensities by dividing peak areas with the biomass of the fungus that produced the signals, and we then compared the normalized signal intensities between single cultures and dual cultures to identify metabolites suppressed, stimulated, and newly formed in dual cultures. Metabolites with normalized intensities at least 10-times lower in dual cultures as compared to single cultures were designated suppressed. Some of the metabolic signals observed in single 
cultures were not found in dual cultures, and we refer to this phenomenon as complete suppression. The number of metabolic signals suppressed in dual culture is shown in Table 3. It is evident that among the three interacting species, A.n. had the greatest ability to suppress the metabolites produced by the other fungi in dual culture and that the metabolites of A.n. were the least suppressed by the other fungi. As shown in Table 3, some metabolites were suppressed specifically in dual cultures with one competitor while others were suppressed by both competitors.

Some metabolites accumulated at higher concentrations in the supernatants of dual cultures than in those of single cultures. When signal intensity normalized by biomass increased 10-times or more in a dual culture relative to single culture, the metabolite was referred to as induced. Generally, the number of induced metabolites was low; no induced metabolites were detected in dual cultures of A.n. and G.r. at any sampling time (Table 5).

Many metabolites detected in the supernatants of dual cultures were not found in any single culture. The origin of these metabolites is unknown; we refer to them as new, interaction-specific metabolites (Table 4, see). The largest number of new metabolites was detected in A.n. /F.v. dual cultures followed by F.v. / G.r. dual cultures sampled on day 10. Surprisingly, many new metabolites detected on day 10 were unstable or were degraded or transformed by fungal cultures, because fewer interaction-specific metabolites were detected on day 20 and day 30 than on day 10 . 
Table 2. Fitness of fungal species in single and dual cultures of Aspergillus niger (A.n.), Fusarium verticillioides (F.v.), and Gliocladium roseum (G.r.).

\begin{tabular}{|c|c|c|c|c|c|c|c|c|c|c|}
\hline \multirow[t]{2}{*}{ Days } & \multirow{2}{*}{$\begin{array}{c}\text { Interacting } \\
\text { fungi } \\
\mathrm{i}+\mathrm{j}\end{array}$} & \multicolumn{2}{|c|}{$\begin{array}{c}\text { Relative } \\
\text { biomass in } \\
\text { dual culture } \\
{\left[\mathrm{rB}_{\mathrm{i}(\mathrm{d}, \mathrm{j})}\right]}\end{array}$} & \multirow[t]{2}{*}{$\begin{array}{l}\text { Pairwise } \\
\text { competiti } \\
\text {-veness }\end{array}$} & \multicolumn{2}{|c|}{$\begin{array}{c}\text { Relative biomass } \\
\text { in single culture } \\
{\left[\mathrm{rB}_{\mathrm{i}(\mathrm{s}, \mathrm{j})}\right]}\end{array}$} & \multirow[t]{2}{*}{$\begin{array}{l}\text { Growth } \\
\text { rates in } \\
\text { single } \\
\text { cultures }\end{array}$} & \multicolumn{3}{|c|}{$\begin{array}{l}\text { Index of competitive } \\
\text { fitness in dual cultures } \\
{\left[\mathrm{rF}_{\mathrm{i}(\mathrm{j})}\right]}\end{array}$} \\
\hline & & $\mathrm{i}$ & $\mathrm{j}$ & & $\mathrm{i}$ & $\mathrm{j}$ & & A.n. & F.v. & G.r. \\
\hline \multirow[t]{3}{*}{10} & A.n. + F.v. & 1.12 & 0.88 & $\begin{array}{l}\text { A.n. > } \\
\text { F.v. }\end{array}$ & 1.19 & 0.81 & \multirow{3}{*}{$\begin{array}{l}\text { G.r. }> \\
\text { A.n. }> \\
\text { F.v. }\end{array}$} & -0.06 & +0.09 & \\
\hline & A.n. + G.r. & 1.07 & 0.93 & $\begin{array}{c}\text { A.n. > } \\
\text { G.r. }\end{array}$ & 0.96 & 1.04 & & +0.11 & & -0.11 \\
\hline & F.v. + G.r. & 1.35 & 0.65 & $\begin{array}{l}\text { F.v. > } \\
\text { G.r. }\end{array}$ & 0.77 & 1.23 & & & +0.75 & -0.47 \\
\hline \multirow[t]{3}{*}{20} & A.n. + F.v. & 1.76 & 0.24 & $\begin{array}{l}\text { A.n. > } \\
\text { F.v. }\end{array}$ & 1.12 & 0.88 & \multirow{3}{*}{$\begin{array}{c}\text { G.r. > } \\
\text { A.n. > } \\
\text { F.v. }\end{array}$} & +0.57 & -0.73 & \\
\hline & A.n. + G.r. & 1.24 & 0.76 & $\begin{array}{c}\text { A.n. > } \\
\text { G.r. }\end{array}$ & 0.92 & 1.08 & & +0.35 & & -0.30 \\
\hline & F.v. + G.r. & 0.33 & 1.67 & $\begin{array}{l}\text { G.r. > } \\
\text { F.v. }\end{array}$ & 0.80 & 1.20 & & & -0.59 & +0.39 \\
\hline \multirow[t]{3}{*}{30} & A.n. + F.v. & 1.20 & 0.80 & $\begin{array}{l}\text { A.n. > } \\
\text { F.v. }\end{array}$ & 1.38 & 0.62 & \multirow{3}{*}{$\begin{array}{c}\text { G.r. > } \\
\text { A.n. > } \\
\text { F.v. }\end{array}$} & -0.13 & +0.29 & \\
\hline & A.n. + G.r. & 1.28 & 0.72 & $\begin{array}{l}\text { A.n. > } \\
\text { G.r. }\end{array}$ & 0.78 & 1.22 & & +0.64 & & -0.41 \\
\hline & F.v. + G.r. & 0.09 & 1.91 & $\begin{array}{l}\text { G.r. > } \\
\text { F.v. }\end{array}$ & 0.44 & 1.56 & & & -0.80 & +0.22 \\
\hline
\end{tabular}


Table 3. Number of suppressed metabolites in interacting cultures of Aspergillus niger (A.n.), Fusarium verticillioides (F.v.), and Gliocladium roseum (G.r.) ${ }^{1}$

\begin{tabular}{|c|c|c|c|c|c|c|c|}
\hline \multirow[t]{3}{*}{ Producer } & \multirow[t]{3}{*}{ Competitor } & \multicolumn{6}{|c|}{ Number of suppressed metabolites ${ }^{2}$} \\
\hline & & \multicolumn{2}{|c|}{ Day 10} & \multicolumn{2}{|c|}{ Day 20} & \multicolumn{2}{|c|}{ Day 30} \\
\hline & & $\begin{array}{l}\text {-ve } \\
\text { mode }\end{array}$ & $\begin{array}{l}+\mathrm{ve} \\
\text { mode }\end{array}$ & $\begin{array}{l}\text {-ve } \\
\text { mode }\end{array}$ & $\begin{array}{l}\text { +ve } \\
\text { mode }\end{array}$ & $\begin{array}{c}-\mathrm{ve} \\
\text { mode }\end{array}$ & $\begin{array}{l}\text { +ve } \\
\text { mode }\end{array}$ \\
\hline \multirow{3}{*}{ A.n. } & F.v. & 6 & 7 & 7 & 15 & 9 & 28 \\
\hline & G.r. & 2 & 0 & 2 & 1 & 5 & 11 \\
\hline & F.v. \& G.r. ${ }^{3}$ & 2 & 0 & 1 & 1 & 2 & 8 \\
\hline \multirow{3}{*}{ F.v. } & A.n. & 31 & 56 & 17 & 47 & 13 & 39 \\
\hline & G.r. & 20 & 34 & 13 & 20 & 13 & 35 \\
\hline & A.n. \& G.r. ${ }^{3}$ & 18 & 21 & 8 & 13 & 8 & 19 \\
\hline \multirow{3}{*}{ G.r. } & A.n. & 28 & 31 & 32 & 68 & 36 & 103 \\
\hline & F.v. & 23 & 19 & 25 & 42 & 24 & 36 \\
\hline & A.n. \& F.v ${ }^{3}$ & 21 & 16 & 22 & 37 & 23 & 26 \\
\hline
\end{tabular}

${ }^{1}$ Suppressed metabolites were defined as metabolites with normalized signal intensities in dual cultures at least 10-times lower as compared to single cultures. Metabolites transformed or catabolized by interacting species were not included.

${ }^{2}$ Criteria for analysis were: minimum intensity for negative ionization $75,000 \mathrm{cpm}$, for positive ionization 500,000 cpm; MCQ 0.9; relative standard deviation among replicates < 1; change fold-factor $<0.1$.

${ }^{3}$ Number of metabolites suppressed by both competitors.

Table 4. Interaction-specific metabolites produced by dual cultures of Aspergillus niger (A.n.), Fusarium verticillioides (F.v.), and Gliocladium roseum (G.r.) ${ }^{1}$

\begin{tabular}{|c|c|c|c|c|c|c|}
\hline \multirow{2}{*}{$\begin{array}{l}\text { Interacting } \\
\text { fungi }\end{array}$} & \multicolumn{5}{|c|}{ Number of interaction-specific metabolites } \\
\cline { 2 - 7 } & \multicolumn{2}{|c|}{ Day 10} & \multicolumn{2}{|c|}{ Day 20 } & \multicolumn{2}{c|}{ Day 30 } \\
\cline { 2 - 7 } & -ve mode & +ve mode & -ve mode & +ve mode & -ve mode & +ve mode \\
\hline A.n. + F.v. & 37 & 68 & 1 & 10 & 4 & 7 \\
\hline A.n. + G.r. & 7 & 23 & 6 & 17 & 4 & 18 \\
\hline F.v. + G.r. & 19 & 39 & 17 & 37 & 7 & 31 \\
\hline
\end{tabular}

${ }^{1}$ New metabolites were defined as signals found in dual cultures but not in single cultures.

${ }^{2}$ Criteria for analysis were: minimum intensity for negative ionization 75,000 cpm; for positive ionization 500,000 cpm; MCQ 0.9. Products of biotransformation were excluded. 
Table 5. Metabolites stimulated by competition between Aspergillus niger (A.n), Fusarium verticillioides (F.v.), and Gliocladium roseum (G.r.) ${ }^{1}$

\begin{tabular}{|l|l|c|c|c|c|c|c|}
\hline \multirow{2}{*}{ Producer } & \multirow{2}{*}{} & \multirow{2}{*}{} & & \multicolumn{5}{|c|}{ Nompetitor } & \multicolumn{2}{|c|}{ Day 10} & \multicolumn{2}{c|}{ Day 20 } & \multicolumn{2}{c|}{ Day 30} \\
\cline { 3 - 8 } & & $\begin{array}{c}\text {-ve } \\
\text { mode }\end{array}$ & $\begin{array}{c}\text { +ve } \\
\text { mode }\end{array}$ & $\begin{array}{c}\text {-ve } \\
\text { mode }\end{array}$ & $\begin{array}{c}\text { +ve } \\
\text { mode }\end{array}$ & $\begin{array}{c}\text {-ve } \\
\text { mode }\end{array}$ & $\begin{array}{c}\text { +ve } \\
\text { mode }\end{array}$ \\
\hline A.n. & F.v. & 1 & 0 & 0 & 0 & 0 & 2 \\
\hline F.v. & A.n. & 0 & 4 & 0 & 2 & 0 & 0 \\
\hline F.v. & G.r. & 0 & 1 & 0 & 5 & 1 & 8 \\
\hline G.r. & F.v. & 0 & 1 & 0 & 2 & 0 & 0 \\
\hline A.n. & G.r. & 0 & 0 & 0 & 0 & 0 & 0 \\
\hline G.r. & A.n. & 0 & 0 & 0 & 0 & 0 & 0 \\
\hline
\end{tabular}

${ }^{1}$ Induced metabolites were defined as metabolites with normalized signal intensities at least 10-times larger in dual cultures as compared to single cultures.

${ }^{2}$ Criteria for analysis were: minimum intensity for negative ionization $75,000 \mathrm{cpm}$, for positive ionization 500,000 cpm; MCQ 0.9; relative standard deviation < 1 ; change fold-factor $>10$.

\section{Fumonisin synthesis in dual cultures}

Three signals reduced in dual culture of F.v. with G.r. had $\mathrm{m} / z$ of 722.5, 706 and 706 in positive ionization mode. These three signals, which were found in all cultures containing F.v., originated from the mycotoxin fumonisin B1 (FB1), fumonisin B2 (FB2) and fumonisin B3 (FB3), as proven by comparing the retention time and $\mathrm{MS}^{2}$ spectrum with an authentic standard, respectively. As we can see in Figure 3, the amount of FB1, FB2 and FB3 in the dual culture of F.v. with G.r. were significantly reduced than F.v. single cultures. Fumonisins were detectable after 10 days incubation, and their concentrations increased fast at 20 days then appeared to be slowly. At day 30 the amount of FB1, FB2 and FB3 reached to the highest in F.v. single cultures. In the co-culture of F.v. with G.r., the production of FB1, FB2 and FB3 had the same increase trend as F.v. single culture until 25 days but then showed to be decreased slightly at 30 days. The production of FB1 is always the most abundant among these three fumonisins in F.v. single and dual cultures up to 30 days. 


\section{Degradation of FB1 by G. roseum}

Degradation or biotransformation is the likely reason for the reduction of FBs in F.v. / G.r. dual culture than F.v. single culture. We hypothesis that fumonisin, produced by F.v., which could be degraded by its partner fungus G.r. According to this we incubated G.r. together with pure FB1 instead of FB1 producer F.v.. After 10 days, the co-culture of G.r. with FB1 were analyzed using HPLC-MSMS method, the result showed that the amount of FB1 kept the same in its co-culture with G.r. under our incubation conditions. No degradation has been detected in this experiment.
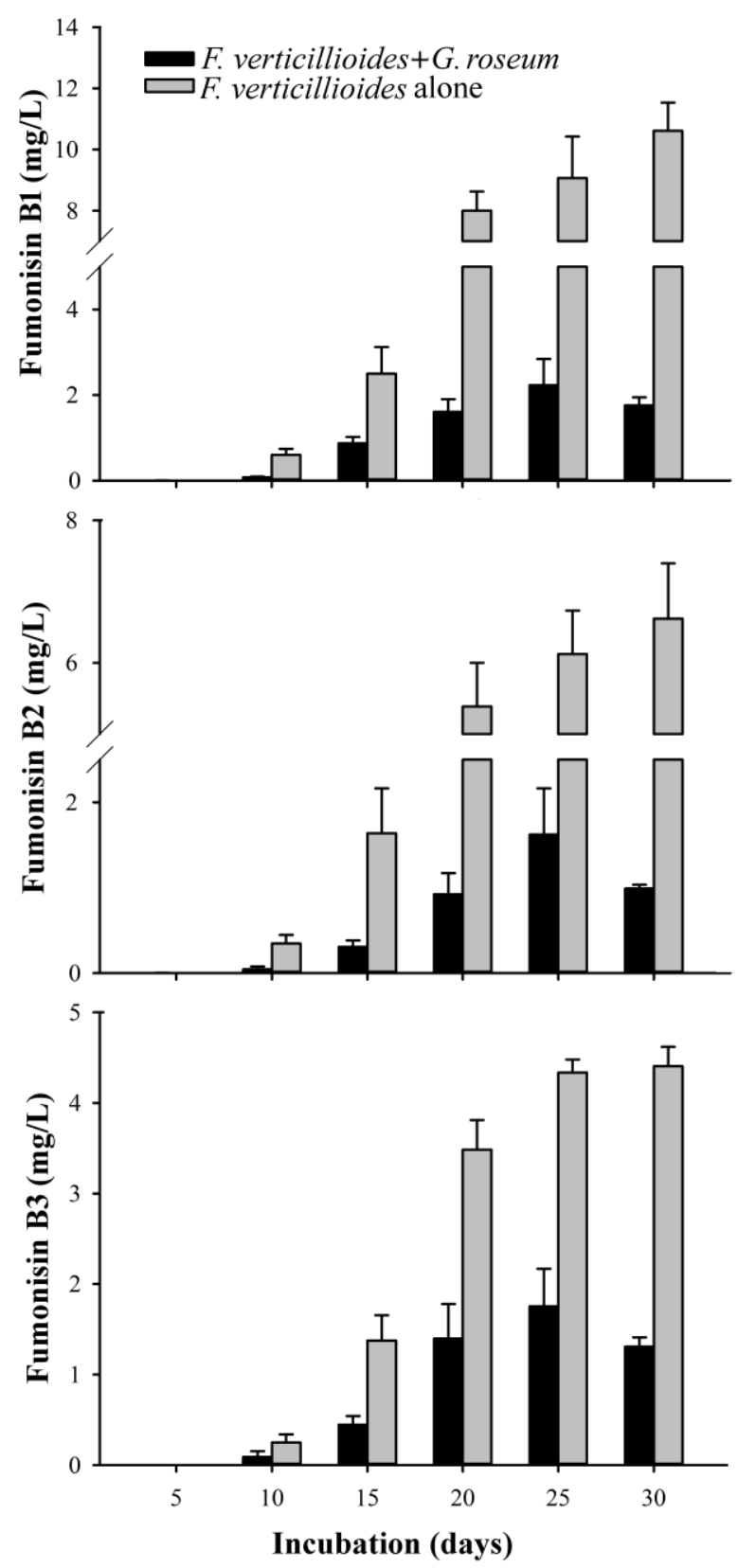

Figure 3. (A) Production of fumonisin B1 by $F$. verticilloides over time in single and dual cultures;

(B) Production of fumonisin B2 by $F$. verticilloides over time in single and dual cultures;

(C) Production of fumonisin B3 by $F$. verticilloides over time in single and dual cultures.

F. verticillioides alone:

F. verticillioides single cultures; F. verticillioides $+G$. roseum: dual cultures of $F$. verticillioides with G. roseum. Error bars indicated the standard error of mean. 


\section{Discussion}

\section{Competitive fitness}

The biomass of both A.n. and G.r. in single cultures increased over the entire cultivation period (Figure 1). The growth of F.v. was slower and the cultures underwent autolysis, perhaps because this phytopathogen and endophyte is not adapted to saprophytic growth under the conditions used. The biomass of G.r. in single culture of was higher than that of A.n. and F.v. at all time points, indicating a high saprophytic fitness of this species even though G.r. is a mycoparasite (Kubicek and Harman, 2007) and entomopathogen (Toledo et al., 2006).

The biomass of F.v. in dual cultures with G.r., declined, suggesting that the mycelium of F.v. was destroyed by mycoparasitism. Interestingly, at day 10, F.v. had generated the similar amount of biomass in dual culture with G.r. as in single culture. The growth of G.r. was strongly suppressed in G.r. / F.v. dual culture before this time point (Figure 1). The fast growth of G.r. after day 10 and the vanishing biomass of F.v. suggest that the temporary growth retardation of G.r. in dual culture with F.v. resulted from physiological self-control rather than from antibiosis. Slow replication and a reduced metabolic rate may have induced an "antibiotic-tolerant" state, as recently shown for Mycobacterium tuberculosis (Baek et al., 2011). We speculate that during the first 10 days, G.r. activated mechanisms that enabled it to cope with interference competition metabolites produced by F.v.. A high number of mutually suppressed metabolites (Table 3) as well as interaction-specific metabolites (Table 4) in dual cultures of G.r. / F.v. corroborate the dominant role of secreted metabolites in this interaction.

G.r. was less successful in dual culture with A.n. than with F.v. (Figure 1). The growth of both fungi was reduced relative to single cultures but the suppression was much more pronounced for G.r. The mycoparasite generated more biomass than A.n. in single cultures but less biomass than A.n. in dual cultures. Its index of competitive fitness was $<-0.30$ at all time points. 
Given that A.n. outcompeted G.r. and that G.r. essentially destroyed F.v., it was surprising that A.n. / F.v. dual cultures did not unequivocally show a dominance of A.n. over F.v. The biomass of A.n. was larger than that of F.v. at all time points (Figure 1) but the growth suppression was similar for both species at day 10 and was larger for A.n. than for F.v. at day 30, resulting in a higher index of competitive fitness for the latter species. The co-incubation with A.n. apparently delayed the senescence and autolysis of F.v. mycelium (Figure 1).

At days 10 and 20, the rankings for competitive fitness were congruent (if $\mathrm{A}>\mathrm{B}$ and $\mathrm{B}>\mathrm{C}$, then $\mathrm{A}>\mathrm{C}$ ). The rankings were A.n. $\approx$ F.v. $>$ G.r. at day 10 and A.n. > G.r. > F.v. at day 20. At day 30, however, the rankings were incongruent in that F.v. > A.n., A.n. > G.r., and G.r. > F.v. This situation is reminiscent of non-transitive systems of three competing species with a rock-paper-scissors relationship (Kerr et al., 2002). Our finding indicates that fungal competitive fitness depends on the competitor, which is in line with observations of Losada et al. (2009) for competing Aspergillus species grown on agar plates. Differences in the ranking among the time points indicate that physiological adaptation altered the competitive fitness of interacting fungal strains in the course of incubation. Understanding how it happened will require the monitoring of fungal biomass at short time intervals.

\section{Metabolic diversity}

According to recent research, however, the synthesis of novel metabolites can be activated by the co-culturing of fungi with other microbes (Oh et al., 2007; Scherlach and Hertweck, 2009; Brakhage and Schroeckh, 2011; Schroeckh et al., 2009). These research efforts focused on the recovery of new structures with potentially useful properties, and the effect of biotic interactions on metabolic diversity was not systematically studied. Metabolic fingerprints generated by HPLC-MS in our work revealed a dramatic and unprecedented increase in the diversity of secreted metabolites in dual fungal cultures. Some of these signals may have resulted from the degradation that escaped detection in mycelium exchange experiments, many of them, however, are 
likely to have originated from new structures and/or from derivatives of known metabolites that have not yet been isolated from these organisms. The low ratio of the number of known secondary metabolites to the number of relevant metabolic pathways found in sequenced fungal genomes is consistent with this hypothesis (Keller et al., 2005).

Intensity of competition has been shown to modulate plant metabolic diversity (Scherling et al., 2010), although direct involvement of plant metabolites in plantplant interactions is limited. Model simulations of microbial interactions in spatially structured communities suggested that antibiosis maintains microbial diversity on an evolutionary time scale (Czaran et al., 2002). Model predictions were confirmed by E. coli populations as long as the interactions and strain dispersal were limited in space (Kerr et al., 2002). Our results suggest an extension of the paradigm of competition-driven maintenance of species/strain diversity to include the competition-driven maintenance of the diversity of secondary metabolites that a fungal species is able to produce. Based on the large increase in the number of metabolic signals obtained from interacting cultures (Table 4), we hypothesize that re-occurring competition has selected for fungal strains that respond to biotic challenges by synthesizing a large set of metabolites with different modes of action, increasing the chance that some of these metabolites will suppress the competitor. Although it is unlikely that a fungus can launch a unique response to each biotic challenge it encounters, different interacting partners triggered different responses. Elucidation of how interacting partners are recognized and how the recognition is translated to control secondary metabolism is a major task for future research in fungal chemical ecology.

Stimulation, suppression, and degradation of secondary metabolites in dual cultures

Contrary to our expectation, only a few of the metabolites detected in single cultures were significantly stimulated (i.e., produced in $\geq 10$-times amounts) in dual cultures (Table 5). In line with the observation of a large number of new metabolites in dual 
cultures, this finding suggests that the synthesis of metabolites involved in interactions with other species is fully suppressed in the absence of these interactions. The tight metabolic control is likely to result from the selection for the minimization of metabolic costs.

Many metabolites accumulated in lower amounts in dual cultures than in single cultures (Table 3). This suppression could be caused by the inhibition of synthesis or secretion, by enzymatic transformation or degradation in the medium, or by uptake of the metabolite by the interacting fungus or adsorption on its cell wall. It is evident from Table 3 that the number of secreted secondary metabolites suppressed in dual cultures differed greatly depending on the species of the producer and the competitor. A particular metabolite from fungus A may be suppressed by fungus B only, by fungus $\mathrm{C}$ only, or by both fungus $\mathrm{B}$ and $\mathrm{C}$. Among the three species, A.n. metabolites were the least suppressed, and this was consistent with biomass data, which indicated that A.n. outcompeted the other two species in dual cultures.

Most metabolic signals observed on day 10 and day 20 had disappeared by the next time point. We hypothesize that catabolism by the producers of the metabolites was the cause. Fungi might be able to take-up their own secondary metabolites and use them as a source of energy. The phenomenon has rarely been documented (i.e., Doyle and Marth, 1978; Savard and Miller, 1992). Our results indicate that these observations may be examples of a widespread yet inadequately studied ability of fungi to scavenge the secondary metabolites they produce.

\section{Inhibition of fumonisin synthesis in $F$. verticillioides by $G$. roseum}

The amount of fumonisins, which are produced by F.v., were reduced in F.v. /G.r. dual culture than in F.v. single culture as a result of co-culturing, the question is still remain as to its biological mechanism. Depend on the results of no biotransformation of FB1 has been done by G.r. suggested that the degradation or biotransformation was not the reason of the fumonisin reduction in F.v. / G.r. dual culture. Therefore, the likely reason of the reduction of FBs in F.v. / G.r. dual culture would be the inhibition of the 
growth of F.v. by the mycoparasitic fungus G.r. Therefore we compared the biomass of F.v. in co-culture with G.r. and its single culture (Figure 1), the results revealed that G.r. can inhibit the growth of F.v., the producer of fumonisins, which lead to the reduction of fumonisins in F.v. / G.r. dual culture. In addition, we did not normalized fumonisins by biomass of F.v., due to the DNA of F.v. were destoried in the dual culture with G.r. after 20 days, however, the already produced fumonisin still kept in the culture supernatants. According to this, the normalization of fumonisins by biomass was not included in this work.

The effect of co-incubation of F.v. with Aspergillus spp. on fumonisin production was studied by Velluti et al. (2000) and Marin et al. (2001), the fumonisin concentrations were not normalized by fungal biomass as well. Depending on the conditions, fumonisin levels in mixed cultures were stimulated or suppressed, but the effects were much less dramatic than in our dual cultures with G.r. Rodriguez Estrada et al. (2011) observed a slight suppression of fumonisin levels normalized by biomass in interactions between F.v. and U. maydis. They speculated that fumonisins may have limited the growth of $U$. maydis in dual cultures with F.v., but except for one publication from the South African Medical Research Council, which is difficult to access (Keyser et al., 1999; Leslie et al., 2008), the effect of fumonisin on fungi has not been studied systematically.

Inhibition of the synthesis of fumonisins by G.r. indicates that the application of G.r. as a biological control agent against F.v. may reduce the mycotoxin levels in the commodity. But it is generally recognized that expression of antagonism by a microorganism towards a pathogen in culture medium cannot be regarded as evidence the microorganism will control the pathogen in the field (Reddy and Hynes, 1994). We should check if G.r. could also be used as biological control agent in the field experiment. 


\section{References}

Adejumo, T.O., Hettwer, U., Karlovsky, P., 2007. Survey of maize from south-western Nigeria for zearalenone, alpha- and beta-zearalenols, fumonisin B1 and enniatins produced by Fusarium species. Food Addit. Contam. 24, 993-1000.

Baek, S.H., Li, A.H., Sassetti, C.M., 2011. Metabolic regulation of mycobacterial growth and antibiotic sensitivity. PLoS Biol. 9, e1001065. doi:10.1371/journal.pbio.1001065.

Brakhage, A.A., Schroeckh, V., 2011. Fungal secondary metabolites - strategies to activate silent gene clusters. Fungal Genet. Biol. 48, 15-22.

Brandfass, C., Karlovsky, P., 2006. Simultaneous detection of Fusarium culmorum and F. graminearum in plant material by duplex PCR with melting curve analysis. BMC Microbiol. DOI 10.1186/1471-2180-6-4

Charusanti, P., Fong, N.L., Nagarajan, H., Pereira, A.R., Li, H.J., Abate, E.A., Su, Y.X., Gerwick, W.H., Palsson, B.O., 2012. Exploiting adaptive laboratory evolution of Streptomyces clavuligerus for antibiotic discovery and overproduction. PLoS ONE 7, e33727. doi:10.1371/journal.pone.0033727.

Child, C.J., Shoolingin-Jordan, P.M., 1998. Inactivation of the polyketide synthase, 6-methylsalicylic acid synthase, by the specific modification of Cys-204 of the b-ketoacyl synthase by the fungal mycotoxin cerulenin. J. Biochem. 330, 933-937.

Cooney, J.M., Lauren, D.R., di Menna, M.E., 2001. Impact of competitive fungi on trichothecene production by Fusarium graminearum. J. Agric. Food Chem. 49, $522-526$.

Czárán, T.L., Hoekstra, R.F., Pagie, L., 2002. Chemical warfare between microbes promotes biodiversity. Proc. Natl. Acad. Sci. 99, 786-790.

Doyle, M., Marth, E., 1978. Aflatoxin is degraded by mycelia from toxigenic and nontoxigenic strains of Aspergilli grown on different substrates. Mycopathologia 63, 145-153.

Karlovsky, P., 2008. Secondary metabolites in soil ecology. In: Secondary metabolites 
in soil ecology (Ed. P. Karlovsky), Springer, 1-19.

Keller, N.P., Turner, G., Bennett, J.W., 2005. Fungal secondary metabolism - from biochemistry to genomics. Nat. Rev. Microbiol. 3, 937-947.

Keller, S.E., Sullivan, T.M., 1996. Liquid culture methods for the production of fumonisin. Adv. Exp. Med. Biol. 392, 205-212.

Kerr, B., Riley, M.A., Feldman, M.W., Bohannan, B.J.M., 2002. Local dispersal promotes biodiversity in a real-life game of rock-paper-scissors. Nature 418, $171-174$.

Keyser, Z., Vismer, H.V., Klaasen, J.A., Snijman, P.W., Marasas, W.F.O., 1999. The antifungal effect of fumonisin B1 on Fusarium and other fungal species. South African Journal of Science 95, 455-458.

Kubicek, C.P., Harman, G.E., editors, 2007. Trichoderma and Gliocladium Vol 2. 1st ed. Taylor \& Francis. 393 p.

Leslie, J.F., Summerell, B.A., 2008. The Fusarium laboratory manual. John Wiley \& Sons. 403 p.

Losada, L., Ajayi, O., Frisvad, J.C., Yu, J., Nierman, W.C., 2009. Effect of competition on the production and activity of secondary metabolites in Aspergillus species. Medical Mycology. 47, Supplement, S88-S96.

Lutz, M.P., Feichtinger, G., Défago, G., Duffy, B., 2003. Mycotoxigenic Fusarium and deoxynivalenol production repress chitinase gene expression in the biocontrol agent Trichoderma atroviride P1. Appl. Environ. Microbiol. 69, 3077-3084.

Marín, S., Albareda, X., Ramos, A.J., Sanchis, V., 2001. Impact of environment and interactions of Fusarium verticillioides and Fusarium proliferatum with Aspergillus parasiticus on fumonisin B1 and aflatoxins on maize grain. Journal of the Science of Food and Agriculture 81, 1060-1068.

O’Brien, J., Wright, G.D., 2011. An ecological perspective of microbial secondary metabolism. Curr. Opin. Biotechnol 22, 552-558.

Oh, D.C., Kauffman, C.A., Jensen, P.R., Fenical, W., 2007. Induced production of emericellamides A and B from the marine-derived fungus Emericella sp. in 
competing co-culture. J. Nat. Prod. 70, 515-520.

Omura, S., Arai, N., Yamaguchi, Y., Masuma, R., Iwai, Y., 2000. Argifin, a new chitinase inhibitor, produced by Gliocladium sp FTD-0668 - I. taxonomy, fermentation and biological activities. J. Antibiot. 53, 603-608.

Ramakrishna, N., Lacey, J., Smith, J.E., 1996. The effects of fungal competition on colonization of barley grain by Fusarium sporotrichioides on $\mathrm{T}-2$ toxin formation. Food Addit. Contam. 13, 939-948.

Ratzinger, A., Riediger, N., von Tiedemann, A., Karlovsky, P., 2009. Salicylic acid and salicylic acid glucoside in xylem sap of Brassica napus infected with Verticillium longisporum. J. Plant Res. 122, 571-579.

Reddy, M.S., Hynes, R.K., 1994. Relationship between in vitro growth inhibition of pathogens and suppression of pre-emergence damping-off and post-emergence root rot of white beans seedlings in the greenhouse by bacteria, Can. J. Microbiol. 40, 113-119.

Rodriguez Estrada, A.E., Hegeman, A., Kistler, H.C., May, G., 2011. In vitro interactions between Fusarium verticillioides and Ustilago maydis through real-time PCR and metabolic profiling. Fungal Genet. Biol. 48, 874-885.

Savard, M.E., Miller, J.D., 1992. Characterization of fusarin F, a new fusarin from Fusarium moniliforme. J. Nat. Prod. 55, 64-70.

Scherlach, K., Hertweck, C., 2009. Triggering cryptic natural product biosynthesis in microorganisms. Org. Biomol. Chem. 7, 1753-1760.

Scherling, C., Roscher, C., Giavalisco, P., Schulze, E.D., Weckwerth, W., 2010. Metabolomics unravel contrasting effects of biodiversity on the performance of $\begin{array}{lllll}\text { individual plant } & \text { Species. }\end{array}$ e12569.doi:10.1371/journal.pone.0012569

Schroeckh, V., Scherlach, K., Nützmann, H.W., Shelest, E., Schmidt-Heck, W., Schuemann, J., Martin, K., Hertweck, C., Brakhage, A.A., 2009. Intimate bacterial fungal interaction triggers biosynthesis of archetypal polyketides in Aspergillus nidulans. Proc. Natl. Acad. Sci. 106, 14558-14563.

Sirenko, L.A., Malyarevskaya, A.Y., Birger, T., Kirpenko, Y.A., 1979. Ecological 
metabolites. In: Krasnov EV (ed) Vzaimodeistne Vodoi Zhiuym Veshchestuom, vol 2. Izdania, Moscow, pp 48-56.

Toledo, A.V., Virla, E., Humber, R.A., Paradell, S.L., Lastra, C.C.L., 2006. First record of Clonostachys rosea (Ascomycota: Hypocreales) as an entomopathogenic fungus of Oncometopia tucumana and Sonesimia grossa (Hemiptera: Cicadellidae) in Argentina. J. Invertebr. Pathol. 92, 7-10.

Utermark, J., Karlovsky, P., 2007. Role of zearalenone lactonase in protection of Gliocladium roseum from fungitoxic effects of the mycotoxin zearalenone. Applied and Environmental Microbiology 73, 637-642.

Velluti, A., Marín, S., Bettucci, L., Ramos, A.J., Sanchis, V., 2000. The effect of fungal competition on colonization of maize grain by Fusarium moniliforme, $F$. proliferatum and $F$. graminearum and on fumonisin B1 and zearalenone formation. Int. J. Food Microbiol. 59, 59-66.

Wicklow, D.T., 1981. Interference competition and the organization of fungal communities. In: The fungal community, its organization and role in the ecosystem. DT Wicklow and GC Carroll (Eds.). Marcel Dekker. New York. pp. $351-375$.

Windig, W., Phalp, J.M., Payne, A.W., 1996. Noise and background reduction method for component detection in liquid chromatography/mass spectrometry. Anal. Chem. 68, 3602-3606. 


\title{
Chapter 3: Production and antifungal activity of fusaric acid derivatives from Fusarium verticillioides co-cultured with
} Gliocladium roseum

\author{
Yi Kuang, Kirstin Scherlach, Petr Karlovsky*
}

\begin{abstract}
The fungal co-cultivation recently has been considered as an effective method to obtain novel metabolites. However, the effect of fungal interactions on secondary metabolites production is poorly understood. A pathogen Fusarium verticillioides and mycoparasitic fungus Gliocladium roseum were co-cultivated. DNA of F. verticillioides was determined by real-time PCR and secreted metabolites were analyzed by HPLC-MS and HPLC-DAD. Co-cultures of $F$. verticillioides with G. roseum resulted in isolation of two new compounds (2 and $\mathbf{3})$ together with two known fusaric acid derivatives. All these isolated metabolites were produced specifically in F. verticillioides and G. roseum co-cultures. Compound 2 was identified as 4-(5-butylpicolinamido) butanoic acid and compound $\mathbf{3}$ was methyl 4-(5-butylpicolinamido) butanoate on the basis of detailed spectroscopic analysis (mass spectrometry and one- and two-dimensional NMR experiments). Compound 2 and $\mathbf{3}$ are fusaric acid biotransformation products by $G$. roseum. The isolated compounds were evaluated for in vitro antifungal activities against Botrytis cinerea and Aspergillus niger. The bioassay showed that the fusaric acid derivatives were less toxic than FA; compound 2 was the least toxic FA derivative, which indicated the detoxification of fusaric acid is involved in fungal interactions.
\end{abstract}




\section{Introduction}

In modern natural product chemistry, single-strain culture is the standard method for cultivating microorganisms. However, the growth conditions of the culture in a flask are significantly different from those in the natural environment where the microorganisms interact with each other (Onaka et al., 2011). To overcome this limitation, many new fermentation methods have been developed, one of them is mixed culture fermentation, the co-culture of two or more microbes (Zuck et al., 2011). According to genomic studies, microbes surviving in the competitive natural environments require potential interesting gene clusters for expressing bioactive secondary metabolites which likely are not expressed or remain silent under standard laboratory single-strain culture condition (Pettit, 2009; Slattery et al., 2001; Schroeckh et al., 2009). Several investigations into natural products which have been obtained from co-culture experiments indicated that the microbes interactions could trigger the production of bioactive secondary metabolites contributing to survival in a competitive environment (Bertrand et al., 2013). Culturing two or more different microbial strains was shown as an effective method to obtain novel molecules ( $\mathrm{Li}$ et al., 2011), change the yields of known metabolites (Oh et al., 2007) and generate the analogues of known metabolites by combine pathways (Degenkolb et al., 2002). Interactions among fungi are dominated by competition. The major mechanism of interference competition is thought to be antibiosis that is caused by secondary metabolites. It has been reported that the originally silent gene clusters that are involved in secondary metabolite biosynthesis could be induced by biotic interactions (Schroeckh et al., 2009; Scherlach and Hertweck, 2009). However, most reports of fungal co-cultivation mainly focused on the discovery of novel metabolites with potentially useful properties (Cueto et al., 2001; Zuck et al., 2011; Li et al., 2011; Bertrand et al., 2013), while the effect on fungal secondary metabolites biosynthesis is poorly understood. Furthermore, the origin and the biological role of the structures and the effect of biotic interactions on metabolic diversity were not systematically studied. We introduced a method to evaluate interaction mechanisms between Fusarium verticillioides and Gliocladium roseum, and monitored the changes of metabolites that are affected in the interaction cultures (Chapter 1).

F. verticillioides (Sacc. Nirenberg, syn. Moniliforme Sheldon), formerly known as F. moniliforme, is common on different host plants. It is a well known causative agent 
of ear and kernel rotting of maize (Presello et al., 2008; Sanchez-Rangel et al., 2005), but also occurs as endophytic fungus. F. verticillioides is famous to produce mycotoxin fumonisins and some other biologically active metabolites, including the toxins fusaric acid and fusarins (Brown et al., 2012). The occurrence of fumonisins B in infected maize and maize products have been reported to cause variety of diseases in animals after eating the contaminated feeds, such as leukoencephalomalacia in horses and pulmonary edema syndrome in swine (Ross et al., 1990) and plays a role in human esophageal cancer and neural tube defects (Marasas et al., 2004). Control of fumonisin contamination in maize has become a priority in food safety research. Fusaric acid is a phytotoxin which was first reported in 1934 produced by Gibberella fujikuroi on rice plants (Yabuta et al., 1934). Later on, it has been reported to exist in F. moniliforme (Burmeister et al., 1985). As a plant toxin fusaric acid is associated with damping-off and root rot diseases of many vegetables and crops, it is also considered as a mild toxin to the mammals and brine shrimp (Capasso et al., 1996).

The fungus G. roseum (syn. Clonostachys rosea), is widely distributed in different environments as a nonpathogenic parasite of plants. G. roseum could associate with other organisms such as fungi, several nematodes and plants (Sutton et al., 1997; Zhang et al., 2008). As a destructive mycoparasite, G. roseum represents as an efficient biological control agent against several plant pathogenic fungi, including Botrytis spp. and Fusarium spp. (Li et al., 2002; Xue, 2002). It has been reported that, G.roseum could detoxify the mycotoxin zearalenone by producing a zearalenone-specific lactonase which catalyzes the hydrolysis of zearalenone, followed by a spontaneous decarboxylation. The enzyme converts zearalenone to cleavage product to protect itself from the toxic environments (Utermark and Karlovsky, 2007).

The aim of this study was to identify the interaction-specific metabolites from co-cultures of $F$. verticillioides with $G$. roseum. The activity of the co-cultured strains was monitored by HPLC-MS metabolic profiling and the relative fungal biomass assessment. The toxin production in mono- and co-cultures was investigated to obtain insights about potential biotransformation during their interaction. 


\section{Materials and methods}

\section{General experimental procedure}

Mycelium from liquid culture was filtered, then freeze-dried for real-time PCR analysis and the fermentation broth was stored at $-20^{\circ} \mathrm{C}$ immediately. Four flasks for each treatment were used as replicates and all experiments were repeated two times at different time periods.

\section{Chemicals}

Methanol (HPLC-grade), ethyl acetate (p.a. grade), cyclo-hexan (p.a grade) were purchased from Carl Roth GmbH \& Co. KG (Karlsruhe, Germany). Acetonitrile (LC-MS grade) and methanol (LC-MS grade) were purchased from Th. Geyer GmbH \& Co. KG (Reniningen, Germany). Acetic acid (LC-MS grade) was purchased from Sigma-Aldrich GmbH (Steinheim, Germany). Diethyl chlorophosphate (97\%, 25g), was purchased from Sigma-Aldrich (Poland). Lithium hydroxide monohydrate $(98.5 \%, 100 \mathrm{~g})$ was purchased from Sigma-Aldrich (USA). $\mathrm{H}-\gamma-\mathrm{Abu}-\mathrm{OtBu} \cdot \mathrm{HCl}(1 \mathrm{~g})$ was purchased from Bachem AG (Switzerland). Fusaric acid was purchased from Tokyo chemical industry Co. LTD (Japan).

\section{Fungal strains}

The fungal strains used for this study were listed in Table 1. The fungi were maintained on potato dextrose agar (Carl Roth $\mathrm{GmbH}$, Karlsruhe, Germany). Spore suspensions were prepared in $25 \%$ glycerol and stored at $-80{ }^{\circ} \mathrm{C}$.

\section{Culture conditions}

Fungal mono- and co-cultures were incubated stationary at $21^{\circ} \mathrm{C}$ in GM7 medium in darkness after inoculation of $10^{5}$ spores $/ \mathrm{mL}$. The co-cultures for the metabolic profiling and fusaric acid quantification were incubated in $100 \mathrm{~mL}$ Erlenmeyer flasks containing $30 \mathrm{~mL} \mathrm{GM7} \mathrm{medium} \mathrm{for} \mathrm{5,} \mathrm{10,} \mathrm{15,} \mathrm{20,} 25$ and 30 days. Four flasks for each treatment were used as replicates and all experiments were repeated two times at different time periods. For the specific secondary metabolites purification, 10 days old 
co-cultures of $G$. roseum with $F$. verticillioides were incubated in 1 L Erlenmeyer flasks containing $300 \mathrm{~mL} \mathrm{GM7} \mathrm{medium,} \mathrm{totally} 80 \mathrm{~L}$ was prepared.

Table 1. Fungal strains used in this work

\begin{tabular}{|c|c|c|}
\hline Species & Strain & Source \\
\hline Fusarium verticillioides & FRCM 8114 & $\mathrm{~A}$ \\
\hline Aspergillus niger & 14.203 & $\mathrm{~B}$ \\
\hline Gliocladium roseum & DSMZ 62726 & $\mathrm{C}$ \\
\hline Gliocladium cibotii & DSMZ 2529 & $\mathrm{C}$ \\
\hline Gliocladium flavo-fuscum & DSMZ 3500 & $\mathrm{C}$ \\
\hline Gliocladium catenulatum & DSMZ 62724 & $\mathrm{C}$ \\
\hline Trichoderma virens & DSMZ 1963 & $\mathrm{C}$ \\
\hline Trichoderma harzianum & $\mathrm{Tu}$ & $\mathrm{D}$ \\
\hline Botrytis cinerea & B05.10 & $\mathrm{E}$ \\
\hline Alternaria alternata f.sp.lycopersici & GOE83 & $\mathrm{F}$ \\
\hline Excserohilum turcicum & IPP 1285 & $\mathrm{~B}$ \\
\hline Phoma lingam & IPP 0695 & $\mathrm{~B}$ \\
\hline Magnaporthe oryzae & IPP 1177 & $\mathrm{~B}$ \\
\hline Verticillium longisporum & VL 143 & $\mathrm{~B}$ \\
\hline
\end{tabular}

A: Fusarium Research Center, PA, USA; B: Division of Phytopathology, University of Göttingen, Germany; C: Deutsche Sammlung von Mikroorganismen und Zellkulturen, Braunschweig, Germany; D: Uniseeds Co. LTD., Bangkok, Thailand; E: Division of Plant Pathology, University of Kaiserslautern, Germany; F: Isolated from tomato plant in Sudan by Azza Siddiq Abbo (2008).

\section{Metabolic profiling by HPLC-ESI-MS}

For non-targeted metabolite analysis, a reverse-phase HPLC system coupled to an electrospray ion trap, Varian 500-MS was used as described previously (Ratzinger et al., 2009). The mobile phase consisted of a binary gradient of $7 \mathrm{mM}$ acetic acid in 
95\% water $/ 5 \%$ acetonitrile (A) and $7 \mathrm{mM}$ acetic acid in methanol (B): 0-5 min 90\% A; 5-30 min from $90 \%$ A to $2 \% \mathrm{~A} ; 30-38 \min 2 \% \mathrm{~A}$; and $38-40 \min 2 \% \mathrm{~A}$ to $90 \% \mathrm{~A}$ at a flow rate of $0.2 \mathrm{~mL} / \mathrm{min}$. This was followed by washing and re-equilibration steps. A quality control sample was injected at the beginning, in the middle, and at the end of each sequence to monitor the stability of the method.

Ionization was done by electrospray both in positive and negative mode with the following parameters (negative/positive mode): needle voltage $-4,500 \mathrm{~V} /+5,000 \mathrm{~V}$, shield voltage $-600 \mathrm{~V} /+600 \mathrm{~V}$, capillary voltage $-/+40 \mathrm{~V}$, drying gas (nitrogen) 25 psi $(172.5 \mathrm{kPa})$ at $250{ }^{\circ} \mathrm{C}$, and nebulizing gas (air) $50 \mathrm{psi}(345 \mathrm{kPa})$. The MS analyzer was operated in full-scan mode, mass range m/z 100-1000, scan speed $5000 \mathrm{Da} / \mathrm{s}$, and three scans averaged. For data acquisition, MS workstation/MS Data Review 6.9 (Varian) was used.

\section{Biomass quantification}

The fungal biomass of $F$. verticillioides in its single and co-cultures was quantified by using real-time PCR. The total DNA was extracted from the freeze dried mycelium with the published method (Brandfass and Karlovsky, 2006) and the quality and quantity of the DNA were estimated by using the electrophoresis of $0.8 \%(\mathrm{w} / \mathrm{v})$ agarose gel described by Nutz et al. (2011). The standard curve was created from the serial dilutions of total pure F. verticillioides genomic DNA. The primer pairs used in this study were VER1 (CTTCCTGCGATGTTTCTCC) and VER2 (AATTGGCCATTGGTATTATATATCTA) which amplify a DNA fragment of $578 \mathrm{bp}$ of the calmodulin gene in F. verticillioides (Mulè et al., 2004).

The qPCR analysis was performed by a thermocycler (CFX384TM Real-Time System, C1000TM Thermal Cycler, BioRad, USA) with 384-wells microplates (Kisker Biotech GmbH, Steinfurt, Germany). The reaction mixture contained with $\mathrm{NH}_{4}$ reaction buffer (16 mM (NH$)_{2} \mathrm{SO}_{4} ; 67 \mathrm{mM}$ Tris-HCl; 0.01\% (v/v) Tween 20, pH 8.8, at $25^{\circ} \mathrm{C}$, Bioline, Lükenwalde, Germany), $0.1 \mathrm{mM}$ concentration of each of the four deoxynucleosidetriphosphates (dNTPs; Bioline, Luckenwalde, Germany), $2.5 \mathrm{mM}$ $\mathrm{MgCl}_{2}, 0.5 \mathrm{U}$ of Taq DNA polymerase (BIOTaq, Bioline, Lükenwalde, Germany), 
$0.3 \mu \mathrm{M}$ concentration of each primer, 0.1 x SYBR Green I (Invitrogen, Karlsruhe, Germany), and $1 \mu \mathrm{L}$ of template DNA. The amplification conditions for F. verticillioides were: $2 \mathrm{~min}$ at $95^{\circ} \mathrm{C}$ of the initial denaturation, 40 cycles of $40 \mathrm{~s}$ denaturation at $94^{\circ} \mathrm{C}, 30 \mathrm{~s}$ at $62^{\circ} \mathrm{C}$, and $40 \mathrm{~s}$ elongation at $72^{\circ} \mathrm{C}$. The final elongation step was performed for $4 \mathrm{~min}$ at $72^{\circ} \mathrm{C}$. Fluorescent data were obtained during each annealing phase for setting up a melting curve at the end of each qPCR assay. The qPCR assay was completed by carrying out a melting curve analysis according to the following protocol: $95^{\circ} \mathrm{C}$ for $1 \mathrm{~min}, 55^{\circ} \mathrm{C}$ for $1 \mathrm{~min}$, and heated to $65^{\circ} \mathrm{C}$, then gradual heating of the samples from $65^{\circ} \mathrm{C}$ to $95^{\circ} \mathrm{C}$ at a rate of $0.05^{\circ} \mathrm{C} / \mathrm{s}$ while the fluorescence was measured constantly.

\section{Analysis of fusaric acid (FA) by HPLC-DAD}

Two $\mathrm{mL}$ of fermentation broth of each sample was filtered through a $0.8-\mu \mathrm{m}$-pore-size filter. The analysis of fusaric acid (FA) was performed by HPLC (Jasco, Japan) linked to a photodiode array detector (Prostar DAD 330, Varian, Germany) recording spectral at $271 \mathrm{~nm}$. A Kinetex C18 column $(50.0 \mathrm{~mm}$ x $2.1 \mathrm{~mm}$, particle size $2.6 \mu \mathrm{m}$, Phenomenex, Germany) was used for separation with gradient elution of $10 \%$ to $100 \%$ methanol in water with addition of $1.2 \%$ phosphoric acid and $0.372 \mathrm{mM}$ $\mathrm{Na}_{2}$-EDTA. The flow rate was $0.2 \mathrm{~mL} / \mathrm{min}$. Seven FA concentrations ranging from $1 \mathrm{ppm}$ to $75 \mathrm{ppm}$ were prepared for calculating the standard calibration curve, which was used for the FA quantification of each sample. Samples exceeding the concentration of the highest standard were diluted 10-times.

\section{Purification of specific metabolites}

Ten days old fermentation broth $(80 \mathrm{~L})$ was separated from the mycelium by filtration. Batches of $20 \mathrm{~L}$ were concentrated to about $5 \mathrm{~L}$ volume in a rotary evaporator at $40^{\circ} \mathrm{C}$ and consequently extracted with $5 \mathrm{~L}$ ethyl acetate for three times. The combined ethyl acetate extract was dried in vacuum to yield $2.3 \mathrm{~g}$ crude extract. The crude extract was dissolved in $500 \mathrm{~mL} \mathrm{MeOH}: \mathrm{H}_{2} \mathrm{O}(8: 2, \mathrm{v} / \mathrm{v})$, and then defatted with an equal volume of cyclohexane two times. The residue of aqueous fraction $(1.5 \mathrm{~g})$ was subjected to normal-phase silica gel flash chromatography (Buchi, Switzerland) eluted with a gradient system of $\mathrm{CH}_{2} \mathrm{Cl}_{2}-\mathrm{MeOH}(0-100 \% \mathrm{MeOH})$, and 6 fractions were collected on 
basis of TLC analyses.

Each fraction was injected in the HPLC-MS using the same method used for metabolic profiling as described before. Fungal interaction specific metabolites mainly occurred in fractions 2, 3 and 4. Accordingly, fraction 2 was subjected to a Sephadex LH-20 column chromatography using $\mathrm{MeOH}$ as eluent to yield four subfractions (Fr.2.1-2.4) on basis of TLC analyses. Each subfraction was analyzed by HPLC-MS, indicating subfraction 2.2 and 2.3 contained the metabolites of interest. Pure compound 1 was obtained from subfraction 2.3 by preparative TLC $\left(\mathrm{CH}_{2} \mathrm{Cl}_{2}: \mathrm{MeOH}=25: 1, \mathrm{v} / \mathrm{v}\right)$. Subfractions 2.2 yielded compounds 2 and 4 . Both were purified by preparative TLC $\left(\mathrm{CH}_{2} \mathrm{Cl}_{2}: \mathrm{MeOH}=20: 1\right.$, v/v), and preparative HPLC (Jasco, Japan) on a Nucleodur C18 pyramid column (Macherey-Nagel) using a $\mathrm{MeOH}-\mathrm{H}_{2} \mathrm{O}$ gradient system (10:90 to $\left.98: 2\right)$ with a flow rate of $3.2 \mathrm{~mL} / \mathrm{min}$ within 40 min. Fraction 3 was subjected to RP-C18 column flash chromatography with $\mathrm{MeOH}: \mathrm{H}_{2} \mathrm{O}$ (8:2) which was followed by Sephadex LH-20 column chromatography using $\mathrm{MeOH}$ to yield pure compound $\mathbf{3}$.

\section{Interactions of $\boldsymbol{F}$. verticillioides or $\boldsymbol{G}$. roseum with other fungal species}

Co-cultures of F. verticillioides or G. roseum with combinations among the 11 tested fungal species (Gliocladium cibotii, Gliocladium flavo-fuscum, Gliocladium catenulatum, Trichoderma virens, Trichoderma harzianum, Botrytis cinerea, Alternaria alternata f.sp.lycopersici, Excserohilum turcium, Phoma lingam, Magnaporthe oryzae, Verticillium longisporum) and their respective single cultures were incubated for 10 days. Culture conditions were the same as described previously. The cultures were filtered, defatted with cyclohexane and extracted with ethyl acetate. The extracts were dried and dissolved in $4 \mathrm{~mL} \mathrm{MeOH} / \mathrm{H}_{2} \mathrm{O}(1: 1, \mathrm{v} / \mathrm{v}) .2 \mathrm{~mL}$ of each sample was filtered through a $0.45-\mu \mathrm{m}$ filter membrane just before analysis. Compound 1-4 were used as authentic standards for identification. The described HPLC-DAD method was used for quantification of FA.

\section{Synthesis of compound 1-4}

Synthesis of 1. A solution of an appropriate FA (1 mmol, $0.179 \mathrm{~g})$ in absolute methanol $(5 \mathrm{~mL})$ was refluxed in the presence of concentrated $\mathrm{H}_{2} \mathrm{SO}_{4}(0.3 \mathrm{~mL})$ for 24 hours. After completion of reaction, the reaction mixture was poured into ice-cold 
water $(20 \mathrm{~mL})$ and neutralized with solid potassium carbonate. The reaction product was extracted with EtOAc $(30 \mathrm{~mL})$ for 3 times, then washed with saturated $\mathrm{NaCl}$ solution. The organic layers were combined and further purified by flash chromatography using $\mathrm{MeOH}: \mathrm{H}_{2} \mathrm{O}$ (20:80 to 100:0), leading to pure 1 (yellow oil, $92 \mathrm{mg}, 48 \%$ yielding).

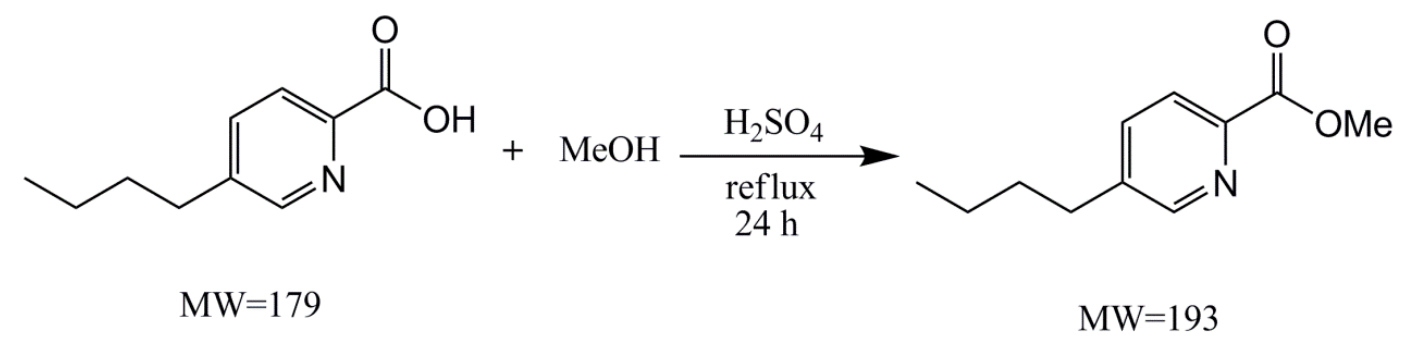

Scheme1. Synthesis of $\mathbf{1}$

Synthesis of 2. A solution of FA (2.0 mmol, $0.358 \mathrm{~g})$ and $10 \mathrm{~mL}$ dry THF was cooled to $0^{\circ} \mathrm{C}$. Into it, $0.29 \mathrm{~mL}(3.0 \mathrm{mmol})$ of diethyl chlorophosphate was added, followed by $0.42 \mathrm{~mL}(3.0 \mathrm{mmol})$ of triethylamine. The reaction was warmed to room temperature and stirred for one hour. To this solution $0.46 \mathrm{~g}(3.0 \mathrm{mmol})$ of $\beta$-alanine ethyl ester hydrochloride was added and followed by $0.42 \mathrm{~mL}(3.0 \mathrm{mmol})$ of triethylamine. The reaction was stirred for 6 hours at room temperature. The reaction mixture was poured into water $(30 \mathrm{~mL})$ and extracted with EtOAc $(30 \mathrm{~mL})$ for 3 times. The combined EtOAc fractions were further purified by flash chromatography using $\mathrm{MeOH}: \mathrm{H}_{2} \mathrm{O}$ (20:80 to 100:0), providing the intermediate ester (yellow oil, $560 \mathrm{mg}$, $87 \%$ yield).

A solution of $500 \mathrm{mg}(1.5 \mathrm{mmol})$ of the intermediate ester prepared as described above of $0.5 \mathrm{~mL}$ of $\mathrm{CF}_{3} \mathrm{COOH}$ in $5 \mathrm{~mL} \mathrm{CH}_{2} \mathrm{Cl}_{2}$, and $1.5 \mathrm{~mL}$ of water was stirred at room temperature for 20 hours. The reaction was poured into water $(30 \mathrm{~mL})$ and extracted with EtOAc $(30 \mathrm{~mL})$ for 3 times. The organic layers were combined, dried (anhydrous $\mathrm{Na}_{2} \mathrm{SO}_{4}$ ) and concentrated in vacuum. The extract was purified by flash chromatography using $\mathrm{MeOH}: \mathrm{H}_{2} \mathrm{O}$ (20:80 to 100:0), providing the pure compound 2 (yellow oil, $282 \mathrm{mg}, 71 \%$ yield). 
<smiles>CCCCc1ccc(C(=O)O)nc1</smiles><smiles>CCCCc1ccc(C(=O)NCCCC(=O)OC(C)(C)C)nc1</smiles><smiles>CCCCc1ccc(C(=O)NCCCC(=O)OC(C)(C)C)nc1</smiles>

Scheme 2. Synthesis of 2

Synthesis of 3. A solution of 2 ( $0.5 \mathrm{mmol}, 0.132 \mathrm{~g})$ in absolute methanol (5 mL) was stirred in the presence of concentrated $\mathrm{H}_{2} \mathrm{SO}_{4}(0.3 \mathrm{~mL})$ at room temperature for 20 hours. After completion of the reaction, the reaction mixture was poured into water $(30 \mathrm{~mL})$ and neutralized with solid $\mathrm{KH}_{2} \mathrm{PO}_{4}$. The reaction products were extracted by EtOAc $(30 \mathrm{~mL}) 3$ times, then washed by saturated $\mathrm{NaCl}$ solution. The combined organic layers were further purified by flash chromatography using $\mathrm{MeOH}: \mathrm{H}_{2} \mathrm{O}$ (20:80 to 100:0), providing pure 3 (yellow oil, $71 \mathrm{mg}, 51 \%$ yield).

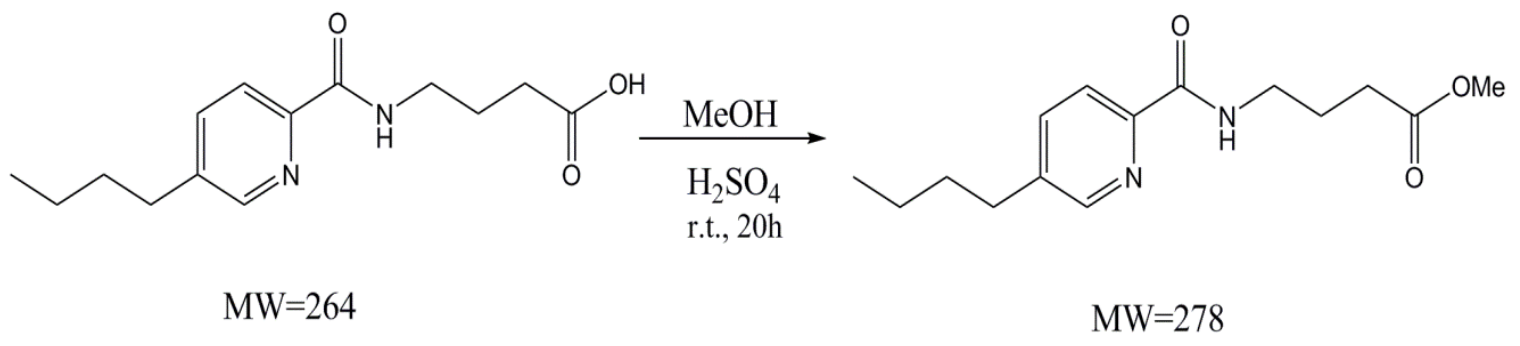

Scheme 3. Synthesis of $\mathbf{3}$ 
Synthesis of 4. FA (0.2 mmol, $35.8 \mathrm{mg})$ was dissolved in methanol $(5 \mathrm{~mL})$ and aqueous $\mathrm{CuSO}_{4}(0.1 \mathrm{mmol}, 15.9 \mathrm{mg})$ were added in $30 \mathrm{~mL}$ water. The reaction mixture was stirred at room temperature for 24 hours. Then the reaction mixture was extracted by EtOAc $(30 \mathrm{~mL})$ for 3 times, the organic layers were combined and evaporated in vacuum. Pure 4 were obtained and crystallized as blue needles ( $25 \mathrm{mg}$, $49 \%$ yield).

\section{FA biotransformation}

For the FA biotransformation experiment, G. roseum, G. catenulatum, G. flavo-fuscum and T. harzianum was cultured in presence of $1 \mathrm{~mL}$ FA solution $(1000 \mathrm{mg} / \mathrm{L})$ at $21^{\circ} \mathrm{C}$ and $28^{\circ} \mathrm{C}$ under the same culture conditions as described previously for 10,20 and 30 days. $1 \mathrm{~mL}$ FA solution without fungi in GM7 medium was prepared parallel as positive control and fungal culture without FA solution was used as negative control. Four flasks for each treatment were used as replicates.

\section{Antifungal bioassay}

The tested fungi in this study were Botrytis cinerea B05.10 and Aspergillus niger 14.203. Antifungal activity was assessed in 96-well microtiter plates with GM7 medium. Serial dilutions of FA and its derivatives were prepared up to $100 \mathrm{mM}$ in DMSO to give seven different concentrations. The tested fungal suspension $\left(10^{4}\right.$ spores $/ \mathrm{mL}$ ) was prepared in GM7 medium. $10 \mu \mathrm{L}$ solution of each tested compound and $190 \mu \mathrm{L}$ of fungal suspension was added into the wells as triplicate. $10 \mu \mathrm{L}$ DMSO were used as negative control. The covered 96-well plate was enclosed in a P flask that was coated with moisture paper and incubated at $25^{\circ} \mathrm{C}$ for one week. Fungal growth in the microtiter plates were checked every 24 hours by reading optical density at $550 \mathrm{~nm}$ with a microplate spectrophotometer ( $\mu$ Quant Universal, BioTekR Instruments, Inc. USA). A four parameter logistic growth curve model was used for curve fitting the determined inhibition values on basis of which the $\mathrm{IC}_{50}$ was estimated (Sigma Plot 11, Systat Software Inc, USA). The micrographs of fungal growth were measured by using an Electron Microscope (Leitz DMRB). 


\section{Results}

\section{Secondary metabolites produced by mixed cultures}

Extraction of co-cultures and single cultures of F. verticillioides and G. roseum were subjected to HPLC-MS. A comparison of the metabolic profiles of co-cultures and single cultures indicated that co-cultivation could affect the production of secondary metabolites (Figure 1). 14 peak signals induced in the interaction cultures were highlighted and considered as new metabolites. For further metabolites identification, four peak signals in the positive ionization mode with $\mathrm{m} / \mathrm{z}$ of 194, 265, 279 and 420, exclusively being detected in dual cultures were selected. The peak signal in positive mode with $\mathrm{m} / \mathrm{z}$ of 265 at $20.5 \mathrm{~min}$ is the dominant new metabolite signal which was induced in the co-culture of $F$. verticillioides and G. roseum. Figure 1 shows the total ion current chromatogram (TIC) of the 10 days old co-culture and single culture of F. verticillioides and G. roseum. The ion traces of compound 2 and $\mathbf{3}$ in 10 days old single and co-cultures are illustrated in Figure 2.

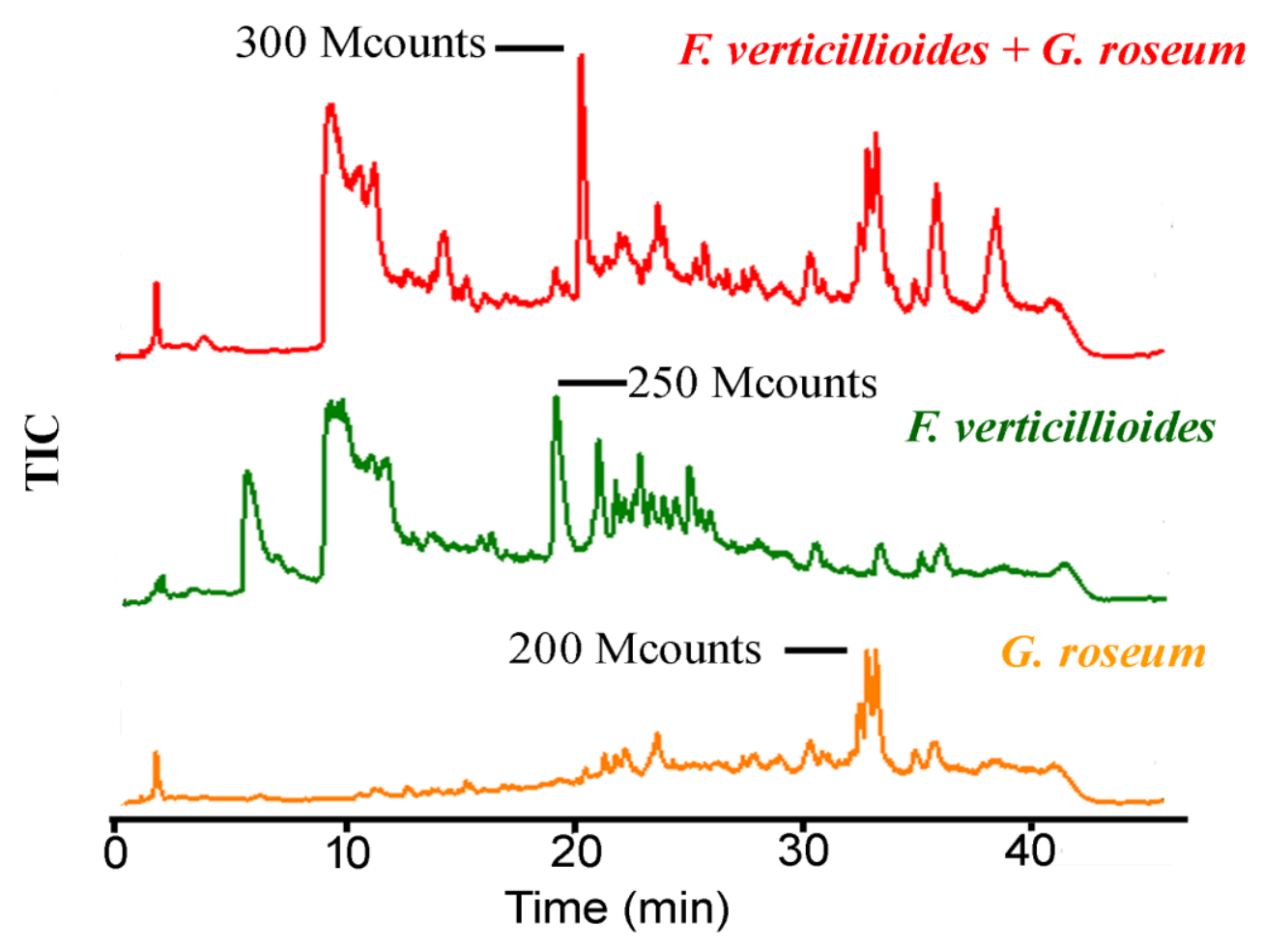

Figure 1. The total ion chromatograms (TIC) of the 10 days old single and co-cultures of Fusarium verticillioides and Gliocladium roseum obtained from HPLC-MS. 

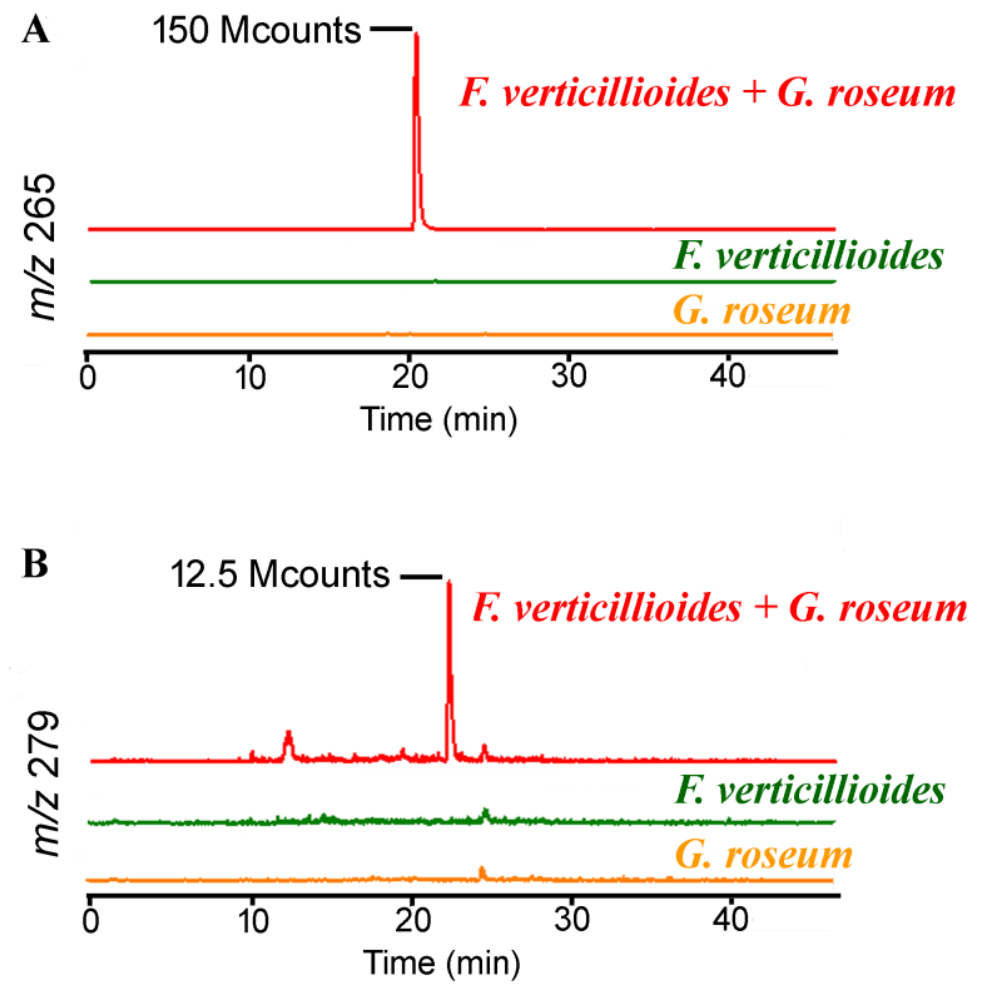

Figure 2. The extract ion chromatograms of compound 2 (A) and compound 3 (B) obtained from HPLC-MS analysis. F. verticillioides: 10 days culture of Fusarium verticillioides; G. roseum: 10 days culture of Gliocladium roseum; F. verticillioides $+G$. roseum: 10 days co-cultures of $G$. roseum with $F$. verticillioides.

\section{Identification of interaction-specific metabolites}<smiles>CCCCc1ccc(C(=O)OC)nc1</smiles><smiles>CCCCc1ccc(C(=O)NCCCC(=O)O)nc1</smiles>

2<smiles>CCCCc1ccc(C(=O)NCCCC(=O)OC)nc1</smiles>

Figure 3. Structures of compound 1-4 
Compound 1 was obtained as white needles. The molecular formula $\mathrm{C}_{11} \mathrm{H}_{15} \mathrm{NO}_{2}$ was established by positive HRESIMS: $m / z=194.1177[\mathrm{M}+\mathrm{H}]^{+}$(calcd. for $\mathrm{C}_{11} \mathrm{H}_{16} \mathrm{NO}_{2}$ 194.1176). The structure determination was performed by means of 1D- and 2D-NMR experiments. A comparison of ${ }^{1} \mathrm{H}-\mathrm{NMR}$ and ${ }^{13} \mathrm{C}-\mathrm{NMR}$ data with published spectral data of FA (Figure 4) suggested that $\mathbf{1}$ had a similar carbon skeleton as FA (Tschesche and Führer, 1978).

The ${ }^{13} \mathrm{C}$-NMR and DEPT spectra displayed 11 carbon signals, which were assigned to two methyls including a methoxy groups, an aliphatic methyl, three methylenes, three aromatic methines, two aromatic quaternary carbons, and one carbonyl carbon. In the ${ }^{1} \mathrm{H}-\mathrm{NMR}$ spectrum of $\mathbf{1}$ (Table 2), in addition to three methylene signals, three aromatic protons $[\delta 7.965$ (d, J=8.0 Hz, H-3), 7.801 (dd, J=2.0, 8.0 Hz, H-4), 8.551 (d, $\mathrm{J}=1.5 \mathrm{~Hz}, \mathrm{H}-6)]$, and a methyl oxygenated methyl proton [3.853 (s, - $\left.\left.\mathrm{OCH}_{3}\right)\right]$ were observed. Furthermore, the HMBC spectrum (Figure 5) of compound 1 showed correlations from H-3 $(\delta 7.965)$ to $\mathrm{C}-5(\delta$ 141.903) and C-11 $(\delta 165.217)$, from H-4 $(\delta$ $7.801)$ to $\mathrm{C}-2(\delta 124.539)$ and C-6 ( $\delta$ 149.786), and from H-6 $(\delta 8.551)$ to C-4 $(\delta$ 136.778). A side chain structure $\left[-\left(\mathrm{CH}_{2}\right)_{3}-\mathrm{CH}_{3}-\right]$ of $\mathbf{1}$ was identified from the ${ }^{1} \mathrm{H}^{-1} \mathrm{H}$ COSY experiments (Figure 5). The position of the side chain on C-5 was confirmed by the HMBC crosspeaks from H-7 to C-4, C-5 and C-6, respectively. This evidence supports the tentative structure of $\mathbf{1}$.

The ${ }^{1} \mathrm{H}$ and ${ }^{13} \mathrm{C}$ NMR spectra of $\mathbf{1}$ were similar to those of FA. The major difference between $\mathbf{1}$ and FA was that the carboxyl group in FA was methyl esterified. $\mathbf{1}$ is an ester derivative of FA. Although $\mathbf{1}$ is known as synthetic product (Renslo and Danheiser, 1998; Tschesche and Führer, 1978), 1 has not been reported previously to occur naturally.

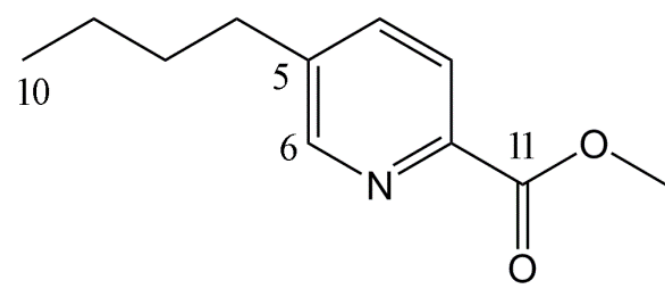

1<smiles>CCCCc1ccc(C(=O)O)nc1</smiles>

fusaric acid

Figure 4. Structures of compound 1 and fusaric acid (FA) 

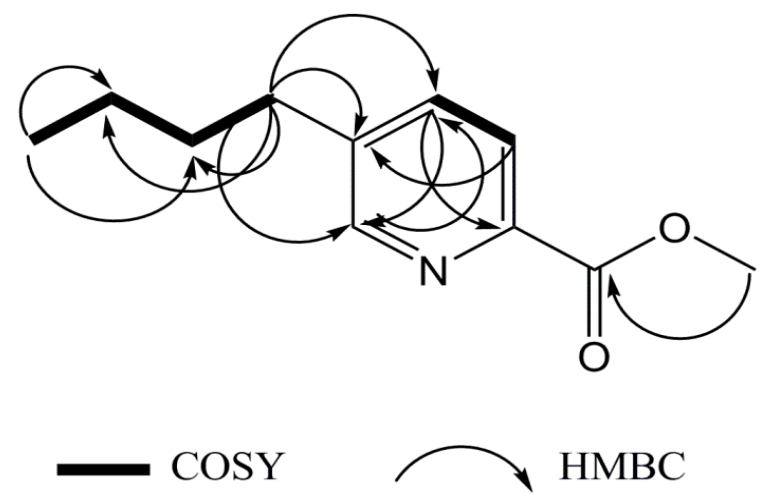

Figure 5. ${ }^{1} \mathrm{H}-{ }^{1} \mathrm{H}$ COSY and selected HMBC correlations of compound $\mathbf{1 .}$

Table 2. ${ }^{1} \mathrm{H}(500 \mathrm{MHz})$ and ${ }^{13} \mathrm{C}(125 \mathrm{MHz}) \mathrm{NMR}$ data of compound $\mathbf{1}$ (DMSO) ${ }^{[\mathrm{a}]}$

\begin{tabular}{llll}
\hline No. & $\boldsymbol{\delta}_{\mathbf{H}}(\boldsymbol{J i n ~ H z})$ & $\boldsymbol{\delta}_{\mathbf{C}}(\mathbf{D E P T})$ & $\mathbf{H M B C}(\mathbf{H} \rightarrow \mathbf{C})$ \\
\hline 1 & & & \\
2 & & $145.140(\mathrm{C})$ & \\
3 & $7.965(\mathrm{~d}, \mathrm{~J}=8.0 \mathrm{~Hz}, 1 \mathrm{H})$ & $124.539(\mathrm{CH})$ & $\mathrm{C}-5, \mathrm{C}-11$ \\
4 & $7.801(\mathrm{dd}, \mathrm{J}=2.0,8.0 \mathrm{~Hz}, 1 \mathrm{H})$ & $136.778(\mathrm{CH})$ & $\mathrm{C}-2, \mathrm{C}-6$ \\
5 & & $141.903(\mathrm{C})$ & \\
6 & $8.551(\mathrm{~d}, \mathrm{~J}=1.5 \mathrm{~Hz}, 1 \mathrm{H})$ & $149.786(\mathrm{CH})$ & $\mathrm{C}-4$ \\
7 & $2.671(\mathrm{t}, \mathrm{J}=7.5 \mathrm{~Hz}, 2 \mathrm{H})$ & $32.372\left(\mathrm{CH}_{2}\right)$ & $\mathrm{C}-4, \mathrm{C}-5, \mathrm{C}-6, \mathrm{C}-8$, \\
& & & $\mathrm{C}-9$ \\
8 & $1.575(\mathrm{~m}, 2 \mathrm{H})$ & $31.653\left(\mathrm{CH}_{2}\right)$ & $\mathrm{C}-5, \mathrm{C}-7, \mathrm{C}-9, \mathrm{C}-10$ \\
9 & $1.288(\mathrm{~m}, 2 \mathrm{H})$ & $21.604\left(\mathrm{CH}_{2}\right)$ & $\mathrm{C}-1, \mathrm{C}-7, \mathrm{C}-8$ \\
10 & $0.888(\mathrm{t}, \mathrm{J}=7.5 \mathrm{~Hz}, 3 \mathrm{H})$ & $13.613\left(\mathrm{CH}_{3}\right)$ & $\mathrm{C}-8, \mathrm{C}-9$ \\
11 & & $165.217(\mathrm{C})$ & \\
$-\mathrm{OCH}$ & $3.853(\mathrm{~s}, 3 \mathrm{H})$ & $52.193\left(\mathrm{CH}_{3}\right)$ & $\mathrm{C}-11$ \\
\hline
\end{tabular}

${ }^{[a]}$ Assigned by COSY, HSQC and HMBC experiments. 
Compound 2 was obtained as yellow oil. The molecular formula $\mathrm{C}_{14} \mathrm{H}_{21} \mathrm{~N}_{2} \mathrm{O}_{3}$ was established by positive HRESIMS, $m / z=265.1546[\mathrm{M}+\mathrm{H}]^{+}$(calcd. for $\mathrm{C}_{14} \mathrm{H}_{21} \mathrm{~N}_{2} \mathrm{O}_{3}$ 265.1547). The structure determination was performed by means of 1D- and 2D-NMR experiments. Through the comparison of ${ }^{1} \mathrm{H}-\mathrm{NMR}$ and ${ }^{13} \mathrm{C}-\mathrm{NMR}$ spectral data with those of known 2-pyridylcarboxamides (Misra and Karanewsky, 1985), it was deduced that $\mathbf{2}$ has a 2-pyridylcarboxamide skeleton.

The ${ }^{13} \mathrm{C}-\mathrm{NMR}$ and DEPT spectra displayed 14 carbon signals, which were assigned as one aliphatic methyl carbon, six methylenes, three aromatic methines, two aromatic quarternary carbons, one carboxamide carbon and one carboxyl carbon. In the ${ }^{1} \mathrm{H}$ NMR spectrum of compound 2 (Table 3), in addition to six methylene signals, three aromatic protons $[\delta 7.927(d, J=7.5 \mathrm{~Hz}, \mathrm{H}-3), 7.781(d d, J=2.5,8.0 \mathrm{~Hz}, \mathrm{H}-4), 8.453$ (d, $J=2.0 \mathrm{~Hz}, \mathrm{H}-6)]$, and a methyl oxygenated methyl proton $\left[3.853\left(s,-\mathrm{OCH}_{3}\right)\right]$ were assigned to in the ${ }^{1} \mathrm{H}-\mathrm{NMR}$ spectrum of $\mathbf{2}$ (Table 3 ).

Inspection of ${ }^{1} \mathrm{H}-{ }^{1} \mathrm{H}$ COSY spectra led to two partial structures a and b (Figure 6). HMBC correlations for H-12 with the quaternary carbon C-11 $(\delta$ C163.9) through a nitrogen atom and the carboxy carbon $\mathrm{C}-15$ to $\mathrm{H}-13$ and $\mathrm{H}-14$ support assuming that partial structure $\mathbf{b}$ could be connected to the amine moiety. Additionally, the HMBC spectrum showed correlations of $\mathrm{H}-7$ and $\mathrm{H}-8$ with $\mathrm{C}-5$, suggesting that partial structure a was attached to C-5.

According to the above analysis, the structure of compound $\mathbf{2}$ was identified as 4-(5-butylpicolinamido) butanoic acid.

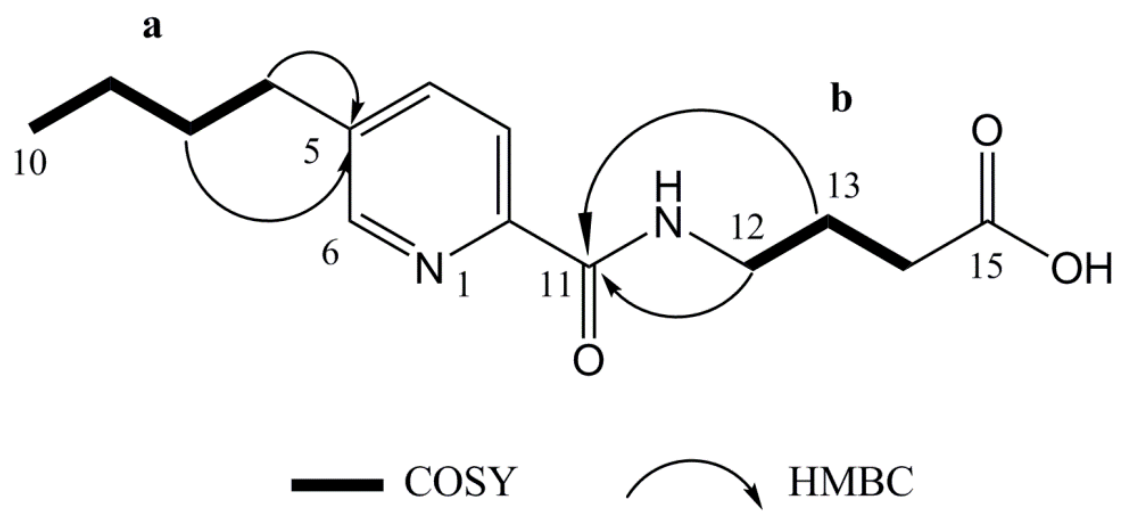

Figure $6 .{ }^{1} \mathrm{H}-{ }^{1} \mathrm{H}$ COSY and selected HMBC correlations of compound 2. 
Table 3. ${ }^{1} \mathrm{H}(500 \mathrm{MHz})$ and ${ }^{13} \mathrm{C}(125 \mathrm{MHz}) \mathrm{NMR}$ data of compound 2 (DMSO) ${ }^{[\mathrm{a}]}$

\begin{tabular}{llll}
\hline No. & $\boldsymbol{\delta}_{\mathbf{H}}(\boldsymbol{J i n} \mathrm{Hz})$ & $\boldsymbol{\delta}_{\mathbf{C}}(\mathbf{D E P T})$ & HMBC $(\mathbf{H} \rightarrow \mathbf{C})$ \\
\hline 1 & & & \\
2 & & $147.944(\mathrm{C})$ & \\
3 & $7.927(\mathrm{~d}, \mathrm{~J}=7.5 \mathrm{~Hz}, 1 \mathrm{H})$ & $121.485(\mathrm{CH})$ & $\mathrm{C}-4, \mathrm{C}-5, \mathrm{C}-11$ \\
4 & $7.781(\mathrm{dd}, \mathrm{J}=2.5,8.0 \mathrm{~Hz}, 1 \mathrm{H})$ & $137.082(\mathrm{CH})$ & $\mathrm{C}-2, \mathrm{C}-3, \mathrm{C}-6, \mathrm{C}-8$ \\
5 & & $140.672(\mathrm{C})$ & \\
6 & $8.453(\mathrm{~d}, \mathrm{~J}=2.0 \mathrm{~Hz}, 1 \mathrm{H})$ & $148.230(\mathrm{CH})$ & $\mathrm{C}-2, \mathrm{C}-3, \mathrm{C}-4, \mathrm{C}-5, \mathrm{C}-7$ \\
7 & $2.650(\mathrm{t}, \mathrm{J}=7.5 \mathrm{~Hz}, 2 \mathrm{H})$ & $31.204\left(\mathrm{CH}_{2}\right)$ & $\mathrm{C}-4, \mathrm{C}-5, \mathrm{C}-6, \mathrm{C}-8, \mathrm{C}-9$ \\
8 & $1.558(\mathrm{~m}, 2 \mathrm{H})$ & $32.556\left(\mathrm{CH}_{2}\right)$ & $\mathrm{C}-5, \mathrm{C}-7, \mathrm{C}-10$ \\
9 & $1.286(\mathrm{~m}, 2 \mathrm{H})$ & $21.605\left(\mathrm{CH}_{2}\right)$ & $\mathrm{C}-7, \mathrm{C}-8, \mathrm{C}-10$ \\
10 & $0.877(\mathrm{t}, \mathrm{J}=8.0 \mathrm{~Hz}, 3 \mathrm{H})$ & $13.619\left(\mathrm{CH}_{3}\right)$ & $\mathrm{C}-8, \mathrm{C}-9$ \\
11 & & $163.934(\mathrm{C})$ & \\
12 & $3.297(\mathrm{dd}, \mathrm{J}=7.0,13.0 \mathrm{~Hz}, 2 \mathrm{H})$ & $38.182\left(\mathrm{CH}_{2}\right)$ & $\mathrm{C}-11, \mathrm{C}-13, \mathrm{C}-14$ \\
13 & $1.750(\mathrm{t}, \mathrm{J}=7.5 \mathrm{~Hz}, 2 \mathrm{H})$ & $24.666\left(\mathrm{CH}_{2}\right)$ & $\mathrm{C}-12, \mathrm{C}-14, \mathrm{C}-15$ \\
14 & $2.228(\mathrm{t}, \mathrm{J}=7.5 \mathrm{~Hz}, 2 \mathrm{H})$ & $31.623\left(\mathrm{CH}_{2}\right)$ & C-12, C-13,C-15 \\
15 & & $174.180(\mathrm{C})$ & \\
$-\mathrm{NH}$ & $8.740(\mathrm{t}, \mathrm{J}=5.0 \mathrm{~Hz}, 1 \mathrm{H})$ & & \\
[aa Assigned by COSY, HSQC and HMBC experiments. &
\end{tabular}

Compound 3 was obtained as yellow powder. The molecular formula $\mathrm{C}_{15} \mathrm{H}_{23} \mathrm{~N}_{2} \mathrm{O}_{3}$ was established by positive HRESIMS, $m / z=279.1701 \quad[\mathrm{M}+\mathrm{H}]^{+}$(calcd. for $\mathrm{C}_{15} \mathrm{H}_{23} \mathrm{~N}_{2} \mathrm{O}_{3}$ 279.1708). The structure determination was performed on basis of 1Dand 2D-NMR experiments. Through the comparison of ${ }^{1} \mathrm{H}-\mathrm{NMR}$ and ${ }^{13} \mathrm{C}-\mathrm{NMR}$ spectral data with compound $\mathbf{2}$, they give the similar data. The key difference between compound 2 and compound $\mathbf{3}$ in ${ }^{13} \mathrm{C}-\mathrm{NMR}$ spectrum was that the chemical shift of C-15. In compound 2, $\delta \mathrm{C}-15$ is 174.180 , but in $\mathbf{3}$ it is 173.053. Moreover, the NMR spectra of 3 indicate the presence of a methoxy carbon $[\delta \mathrm{H} 4.127(s, 3 \mathrm{H})$ and $\delta \mathrm{C}$ 51.2]. Accordingly, $\mathbf{3}$ is the methyl ester of $\mathbf{2}$ (Table 4).

Compound 4 was obtained as blue needle. The molecular formula $\mathrm{C}_{20} \mathrm{H}_{24} \mathrm{~N}_{2} \mathrm{O}_{4} \mathrm{Cu}$ was established by positive HRESIMS, $m / z=420.1114[\mathrm{M}+\mathrm{H}]^{+}$. Structure elucidation was performed by comparison of IR spectra with a known compound ZZF51(A), bis (5-butyl-2-pyridinecarboxylato- $\mathrm{N}_{1}, \mathrm{O}_{2}$ )-copper (Tan et al., 2008). 4 is identical to 
ZZF51(A). Moreover, the comparison of the HPLC-ESI-MS data of the isolated compound $\mathbf{4}$ and the synthesized copper-fusaric acid coordination complex strongly supports this notion. The structure of $\mathbf{4}$ was established as bis-(5-butyl-2-pyridinecarboxylato- $\mathrm{N}_{1}, \mathrm{O}_{2}$ )-copper.

Synthetic compounds. The NMR data of the synthetic compounds were identical with the previous isolated compounds.

Table 4. ${ }^{1} \mathrm{H}(500 \mathrm{MHz})$ and ${ }^{13} \mathrm{C}(125 \mathrm{MHz}) \mathrm{NMR}$ data of compound $\mathbf{3}$ (DMSO) ${ }^{[\mathrm{a}]}$

\begin{tabular}{|c|c|c|c|}
\hline No. & $\delta_{\mathbf{H}}(J$ in Hz $)$ & $\delta_{\mathrm{C}}(\mathrm{DEPT})$ & HMBC $(\mathrm{H} \rightarrow \mathrm{C})$ \\
\hline \multicolumn{4}{|l|}{1} \\
\hline 2 & & $147.890(\mathrm{C})$ & \\
\hline 3 & $7.923(\mathrm{~d}, \mathrm{~J}=6.0 \mathrm{~Hz}, 1 \mathrm{H})$ & $121.490(\mathrm{CH})$ & C-4, C-5, C-11 \\
\hline 4 & $\begin{array}{l}7.794(\mathrm{dd}, \mathrm{J}=1.5,6.5 \mathrm{~Hz}, \\
1 \mathrm{H})\end{array}$ & $137.109(\mathrm{CH})$ & C-2, C-3, C-6, C-8 \\
\hline 5 & & $140.697(\mathrm{C})$ & \\
\hline 6 & $8.461(\mathrm{~d}, \mathrm{~J}=1.5 \mathrm{~Hz}, 1 \mathrm{H})$ & $148.238(\mathrm{CH})$ & C-2, C-3, C-4, C-5,C-7 \\
\hline 7 & $2.664(\mathrm{t}, \mathrm{J}=6.5 \mathrm{~Hz}, 2 \mathrm{H})$ & $31.610\left(\mathrm{CH}_{2}\right)$ & C-4, C-5, C-6, C-8, C-9 \\
\hline 8 & $1.340(\mathrm{~m}, 2 \mathrm{H})$ & $32.552\left(\mathrm{CH}_{2}\right)$ & $\mathrm{C}-5, \mathrm{C}-7, \mathrm{C}-10$ \\
\hline 9 & $1.253(\mathrm{~m}, 2 \mathrm{H})$ & $21.598\left(\mathrm{CH}_{2}\right)$ & C-7, C-8, C-10 \\
\hline 10 & $0.878(\mathrm{t}, \mathrm{J}=6.0 \mathrm{~Hz}, 3 \mathrm{H})$ & $13.632\left(\mathrm{CH}_{3}\right)$ & C-8, C-9 \\
\hline 11 & & $166.933(\mathrm{C})$ & \\
\hline 12 & $\begin{array}{l}3.310(\mathrm{dd}, \quad \mathrm{J}=7.0, \quad 13.0 \\
\mathrm{Hz}, 2 \mathrm{H})\end{array}$ & $38.047\left(\mathrm{CH}_{2}\right)$ & C-11, C-13, C-14 \\
\hline 13 & $1.777(\mathrm{t}, \mathrm{J}=6.0 \mathrm{~Hz}, 2 \mathrm{H})$ & $24.557\left(\mathrm{CH}_{2}\right)$ & C-12, C-14, C-15 \\
\hline 14 & $2.319(\mathrm{t}, \mathrm{J}=6.0 \mathrm{~Hz}, 2 \mathrm{H})$ & $30.794\left(\mathrm{CH}_{2}\right)$ & C-12, C-13,C-15 \\
\hline 15 & & $173.053(\mathrm{C})$ & \\
\hline$-\mathrm{NH}$ & $8.748(\mathrm{t}, \mathrm{J}=5.0 \mathrm{~Hz}, 1 \mathrm{H})$ & & \\
\hline$-\mathrm{OCH}_{3}$ & $4.127(\mathrm{~s}, 3 \mathrm{H})$ & $51.184\left(\mathrm{CH}_{3}\right)$ & $\mathrm{C}-15$ \\
\hline
\end{tabular}


Accumulation of FA and fungal biomass in co-cultures of $F$. verticillioides with G. roseum

The four specific compounds we have isolated from dual cultures of $F$. verticillioides with G. roseum were all derivatives of FA which could be produced by F. verticillioides (Burmeister et al., 1985). Therefore we studied the production of FA in the single and co-cultures of $F$. verticillioides.

In liquid GM7 medium, F. verticillioides started to produce low amounts of FA in single cultures. After 20 days, FA production reached its maximum level, which remained unchanged till 30 days. In the dual cultures of $F$. verticillioides with G. roseum, FA was detectable after 5 days of incubation, and its concentration reached to the maximum after 20 days, but appeared to decrease slowly afterwards (Figure 7A).

The biomass of $F$. verticillioides reached its maximum after 10 days in single cultures and remained unchanged until 30 days (Figure 7B). In dual culture with G. roseum, however, the amount of $F$. verticillioides biomass declined after 25 days. The biomass of $F$. verticillioides was nearly completely destroyed by mycoparasite G. roseum after 30 days.

\section{Origin of FA derivatives and accumulation of FA in co-cultures of F. verticillioides with different fungi}

In order to identify the origin of the FA derivatives and test whether induction of biosynthesis and/or transformation were species-specific, both $F$. verticillioides and G. roseum were incubated with ten further fungal strains, including three Gliocladium and two Trichoderma which are considered to be biocontrol agents (Jensen et al., 2000; Mukherjee et al., 2008). The extracts of each 10-days-old single and co-cultures were analyzed. Compounds 1-4 were found only in co-cultures of $F$. verticillioides and $G$. roseum but not in the co-cultures with the other ten tested fungal species or in their single cultures.

Similarly, the production of FA in co-culture samples with $F$. verticillioides was checked. Figure 8 shows the amount of FA in the $F$. verticillioides single cultures and other co-cultures. The result shows that in the co-cultures of $F$. verticillioides with Gliocladium flavor-fuscum, Gliocladium catenulatum, Trichoderma harzianum and Fusarium culmorum, the amount of FA was strongly reduced, while in the other 
interaction cultures were not.
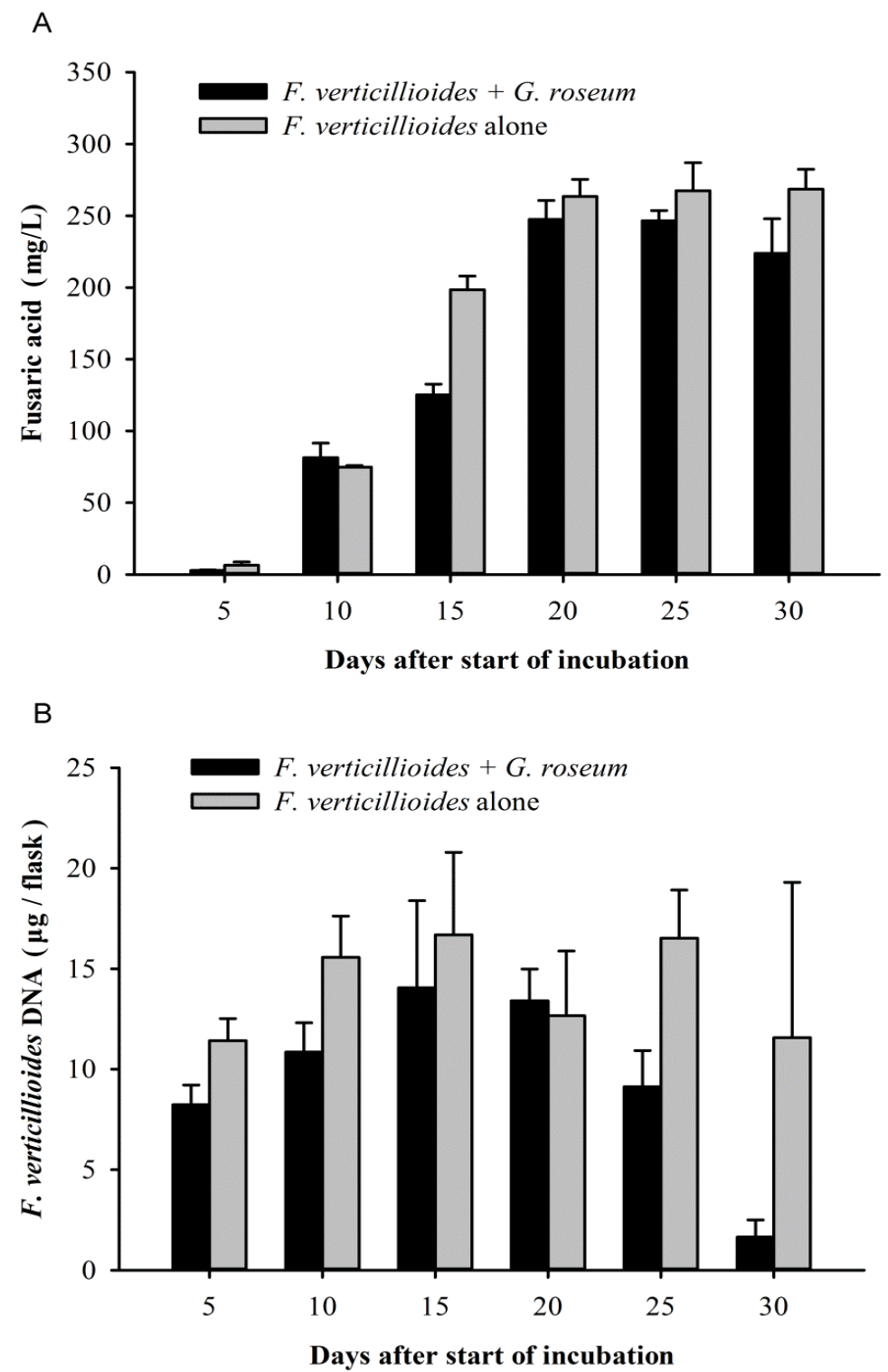

Figure 7. (A) Fusaric acid (FA) in single and co-cultures; (B) DNA of F. verticillioides in single and co-cultures. F. verticillioides alone: Fusarium verticillioides single cultures on GM7 medium; F. verticillioides + G. roseum: co-cultures of Fusarium verticillioides with Gliocladium roseum on GM7 medium. Error bars indicated the standard deviation of mean. 


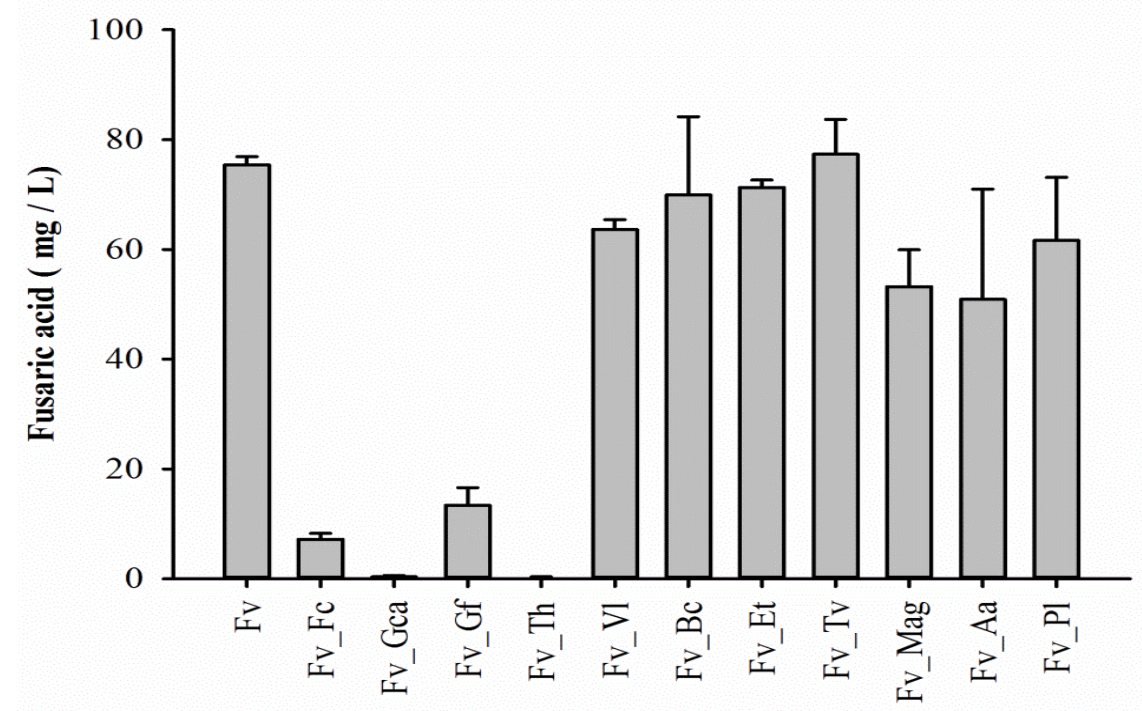

Figure 8. Production of fusaric acid in 10-days-old $F$. verticillioides single and co-cultures. Fv: Fusarium verticillioides single cultures; Fv_Fc: co-cultures of Fusarium verticillioides with Fusarium culmorum; Gca: Gliocladium catenulatum; Gf: Gliocladium flavo-fuscum; Th: Trichoderma harzianum; V1: Verticillium longisporum; Bc: Botrytis cinerea; Et: Excserohilum turcium; Tv: Trichoderma virens; Mag: Magnaporthe oryzae; Aa: Alternaria alternata; Pl: Phoma lingam. Error bars indicated the standard deviation of mean.

\section{Transformation of FA by G. roseum}

To obtain more insight about the origin of the specific metabolites 1-4 from co-cultures of $F$. verticillioides with G. roseum, we suspected these compounds could either originate from biosynthesis pathways in one of the fungi (probably F. verticillioides) induced by co-culturing with the other fungus, or they could be transformation products of FA metabolized by G. roseum. To distinguish between these two possibilities, we incubated $G$. roseum together with pure FA, monitored FA concentration and searched for the FA derivatives (1-4) in culture supernatants up to 30 days. Figure 9 shows that FA concentration remained stable in the positive control without $G$. roseum at both $21^{\circ} \mathrm{C}$ and $28^{\circ} \mathrm{C}$, but in the co-culture with G. roseum it was degraded. The degradation of FA by G. roseum at $28^{\circ} \mathrm{C}$ was faster than $21^{\circ} \mathrm{C}$. At $28^{\circ} \mathrm{C}$ after 20 days the FA was completely degraded by G. roseum. After 30 days, in both temperature conditions FA were completely degraded by G. roseum. HPLC analysis of culture supernatants with MS detection in positive ionization full scan mode 
showed that two peaks, one with $m / z=265$ and another one with $m / z=279$, which correspond to $\mathbf{2}$ and $\mathbf{3}$ were found in all the cultures of G. roseum with FA, but not in the controls. A comparison of retention time and fragmentation spectra with authentic standards of $\mathbf{2}$ and $\mathbf{3}$ confirmed that these two metabolites are the biotransformation products of FA by G. roseum. Figure 10 illustrates FA biotransformation by G. roseum into 2 and 3 up to 30 days at $21^{\circ} \mathrm{C}$ and $28^{\circ} \mathrm{C}$. By contrast, 1 and 4 could not be detected in the cultures of $G$. roseum with FA.
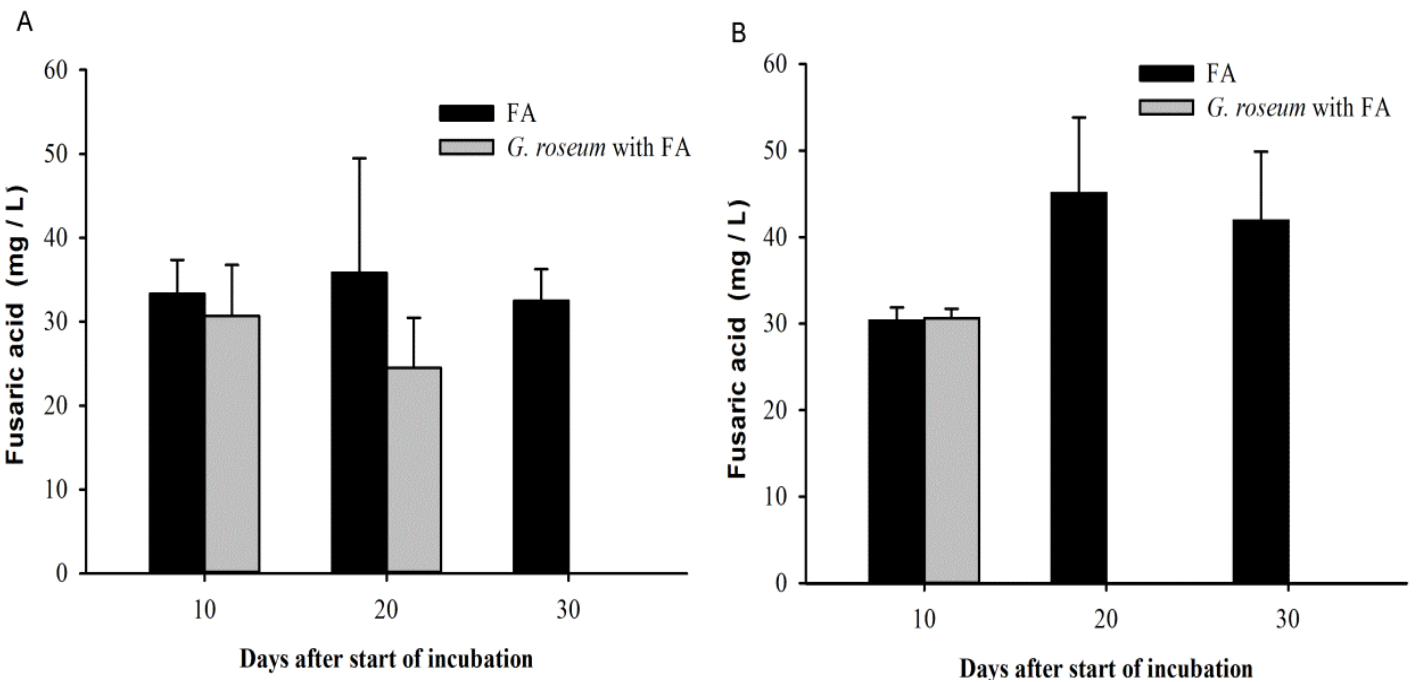

Figure 9. Fusaric acid (FA) degradation by Gliocladium roseum at $21^{\circ} \mathrm{C}$ (A) and $28^{\circ} \mathrm{C}$ (B). FA: fusaric acid in GM7 medium; G. roseum with FA: mix culture of fusaric acid with Gliocladium roseum. Error bars indicated the standard deviation of mean.

A

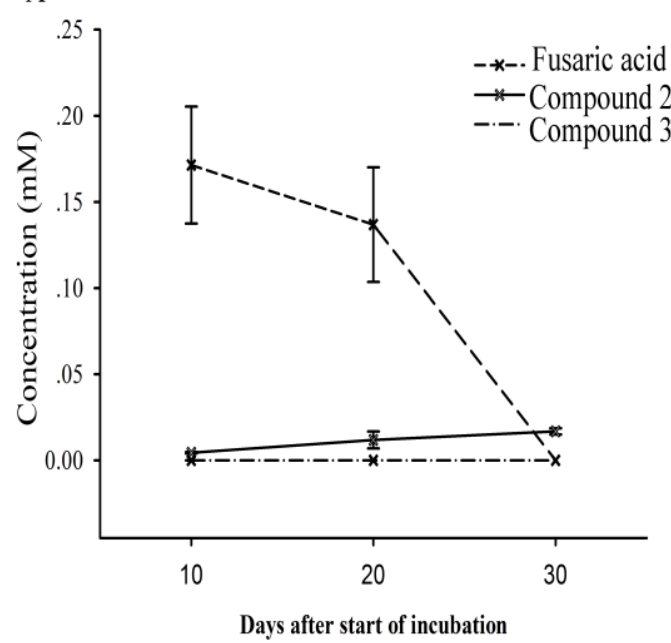

B

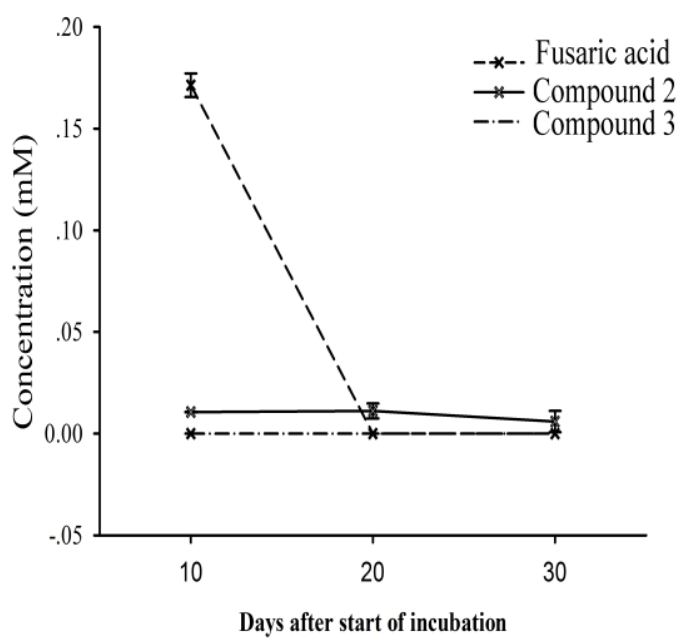

Figure 10. Amount of fusaric acid, compound $\mathbf{2}$ and compound $\mathbf{3}$ in the mix culture of Gliocladium roseum with FA in GM7 medium at $21^{\circ} \mathrm{C}$ (A) and $28^{\circ} \mathrm{C}(\mathrm{B})$. Error bars 
indicated the standard deviation of mean.

\section{Antifungal activity}

Antifungal activity of FA and metabolites 1-3 were explored against Botrytis cinerea and Aspergillus niger (see Figure 11 and Figure 12). The micrographs of A. niger (Figure 11A) show that with $1 \mathrm{mM}$ FA only few spores germinated, while with compound 2 is as same as control. The micrographs of B. cinerea (Figure 12A) show that with $1 \mathrm{mM}$ FA and compound $\mathbf{3}$ spore did not germinate. The antifungal activities of compound 1-3 and FA against $A$. niger and B. cinerea were shown in Table 5. $\mathrm{IC}_{50}$ values shown that for compound $\mathbf{1}$ and $\mathbf{3}$ displayed similar antifungal activities against A. niger and B. cinerea, while the compound $\mathbf{2}$ showed significant less toxicity compared to the positive control FA.

Table 5. Antifungal activity of $\mathbf{1 - 3}$ and fusaric acid (IC50/ mM)

\begin{tabular}{ccc}
\hline \multirow{2}{*}{ Compound } & \multicolumn{2}{c}{ Fungi $\left(\mathbf{I C}_{\mathbf{5 0}} / \mathbf{~ m M}\right)$} \\
\cline { 2 - 3 } & Botrytis cinerea & Aspergillus niger \\
\hline $\mathbf{1}$ & 0.4622 & 1.1098 \\
$\mathbf{2}$ & 2.4083 & 7.5751 \\
$\mathbf{3}$ & 0.5611 & 0.9342 \\
Fusaric acid & 0.2847 & 0.2140 \\
\hline
\end{tabular}

\section{Degradation of FA by G. flavor-fuscum, G. catenulatum and T. harzianum}

Due to the reduction of $\mathrm{FA}$ in the co-cultures of $F$. verticillioides with G. flavor-fuscum, G. catenulatum and T. harzianum, we suspected these fungi could degrade FA. Therefore, we incubated G. flavor-fuscum, G. catenulatum and T. harzianum with pure FA at $28^{\circ} \mathrm{C}$, for monitoring FA concentrations and searching for the compound 1-4 in culture supernatants up to 30 days. Figure 13 shows that FA concentration remained stable in culture supernatants with $G$. catenulatum. The reduced amount of FA in the culture with G. flavor-fuscum and T. harzianum suggest that these two fungi were able to degrade FA. At 30 days, the fungi G. flavor-fuscum and T. harzianum degraded $55 \%$ and $84 \%$ of the amount of FA, respectively. 

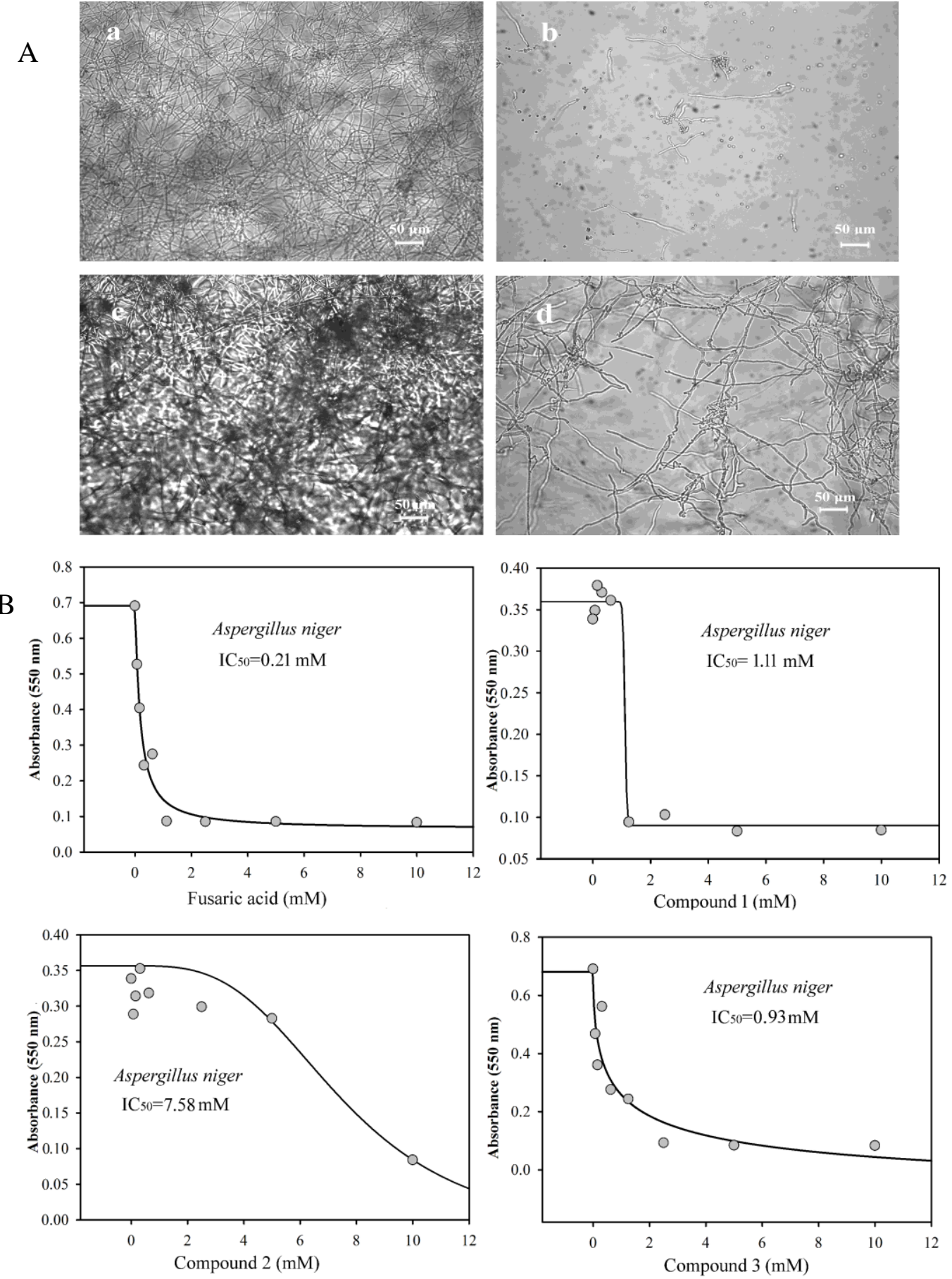

Figure 11. (A) Antifungal activity of fusaric acid (FA) and compound 2-3: a. micrograph of Aspergillus niger, b. micrograph of A. niger treated with FA (1mM), c. micrograph of $A$. niger treated with compound $2(1 \mathrm{mM})$, d. micrograph of $A$. niger treated with compound $3(1 \mathrm{mM})$. (B) Inhibition curves of FA and compound 1-3 for A. niger. Calculated by using four parameter logistic growth curve model (Sigma Plot 11, Systat Software Inc, USA). Growth was measured as absorbance. Each data point is the average of three replications. 
A
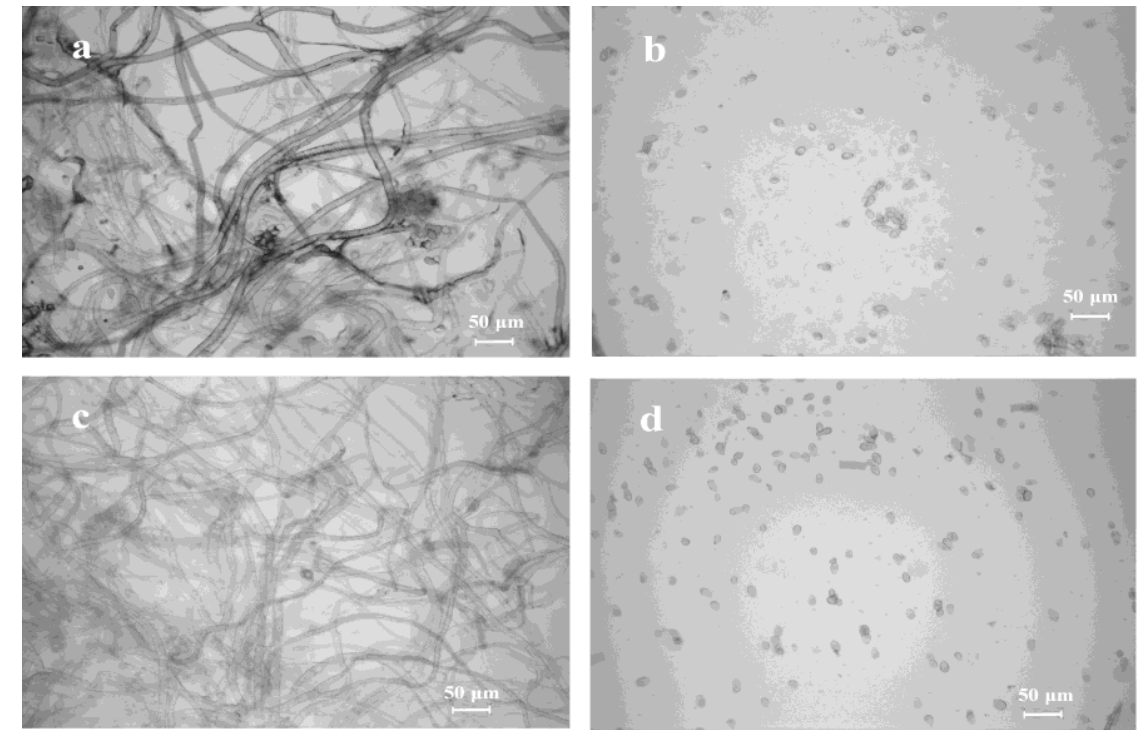

B
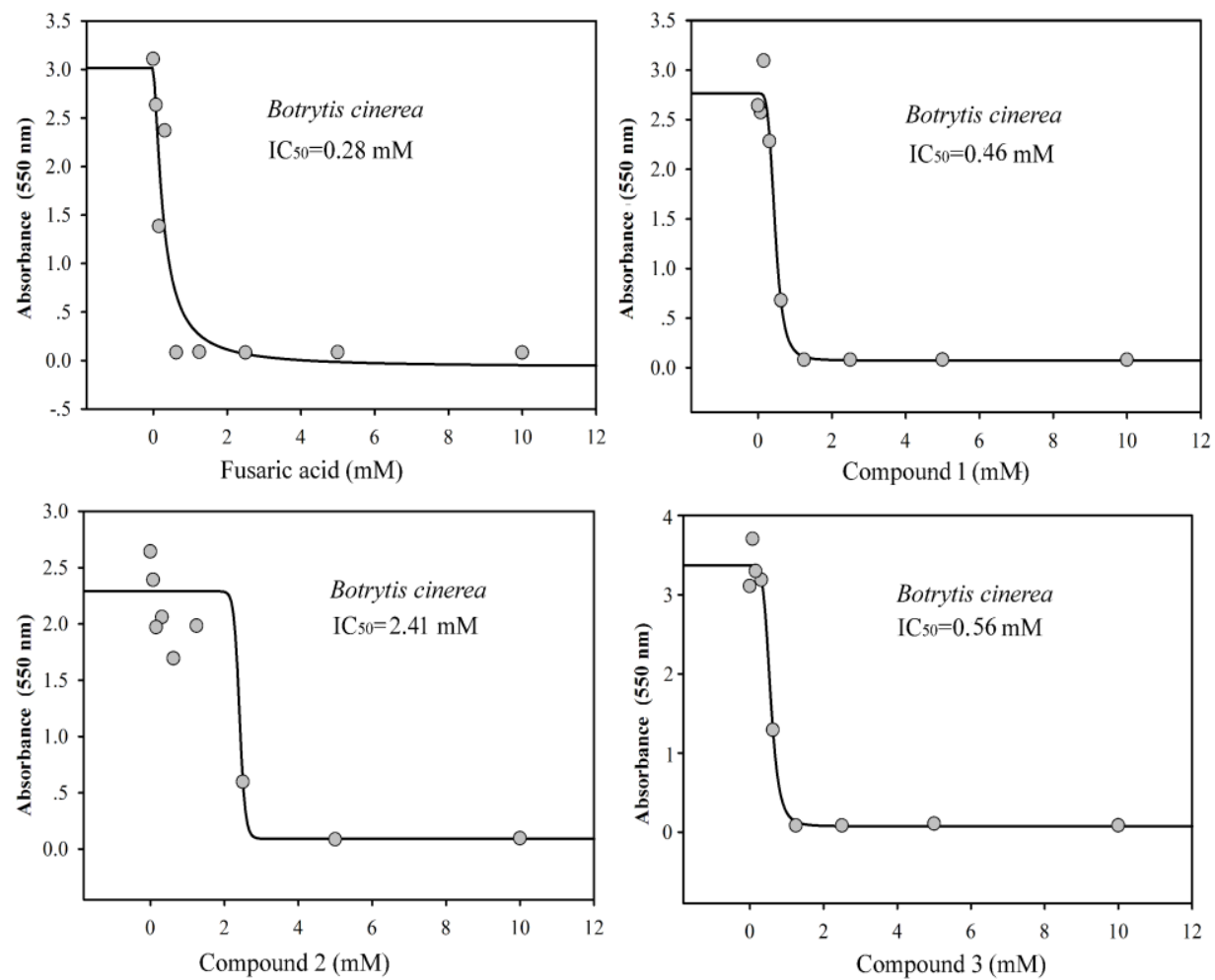

Figure 12. (A) Antifungal activity of fusaric acid (FA) and compound 2-3: a. micrograph of Botrytis cinerea, b. micrograph of B. cinerea treated with FA (1mM), c. micrograph of $B$. cinerea treated with compound $2(1 \mathrm{mM})$, d. micrograph of B. cinerea treated with compound $3(1 \mathrm{mM})$. (B) Inhibition curves of FA and compound 1-3 for B. cinerea. Calculated by using four parameter logistic growth curve model (Sigma Plot 11, Systat Software Inc, USA). Growth was measured as absorbance. Each data point is the average of three replications. 


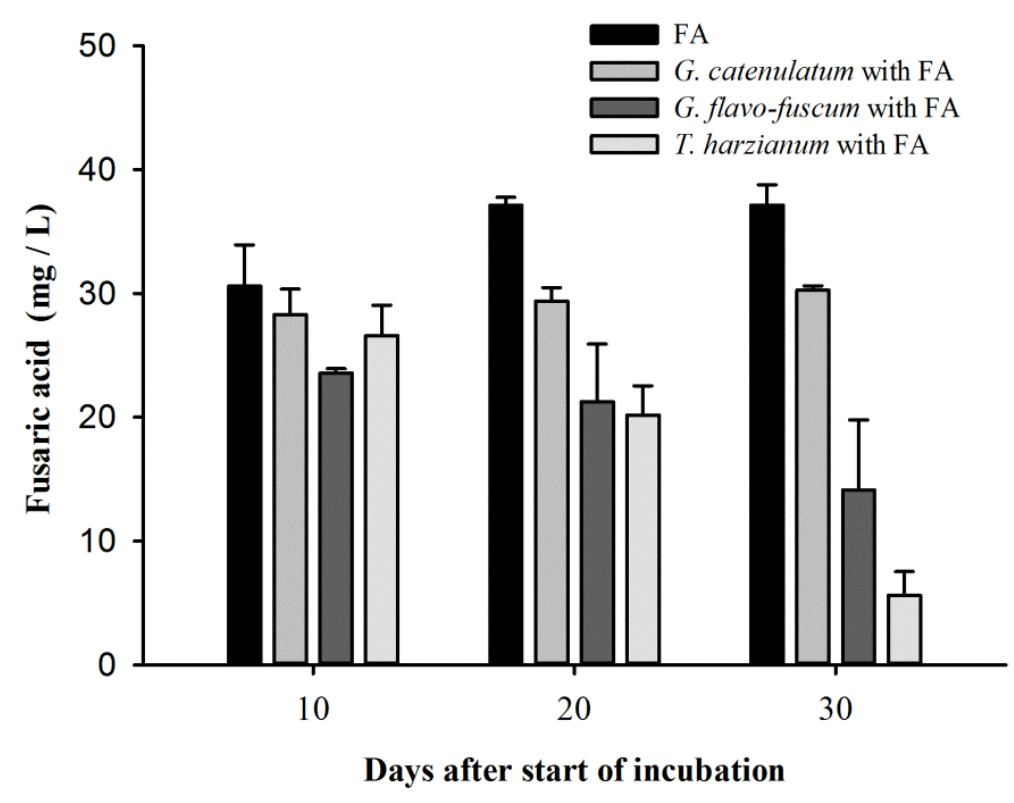

Figure 13. Amount of fusaric acid (FA) in mix cultures of FA with different fungi at $28^{\circ} \mathrm{C}$ in GM7 medium. FA: fusaric acid control; G. catenulatum with FA: mix culture of fusaric acid with Gliocladium catenulatum; G. flavo-fuscum with FA: mix culture of fusaric acid with Gliocladium flavo-fuscum; T. harianum with FA: mix culture of fusaric acid with Trichoderma harianum. Error bars indicated the standard deviation of mean.

\section{Discussion}

In the present study, the production secondary metabolites and the fungal growth affected by the co-cultivation of phytopathogen $F$. verticillioides and mycoparasite G. roseum were investigated systematically. Two novel fungal metabolites, $\mathbf{2}$ and $\mathbf{3}$, together with a new nature product compound 1 and one known FA derivative (4) have been isolated from the co-cultures of $F$. verticillioides and G. roseum for the first time. Surprisingly, we found that compound $\mathbf{2}$ and $\mathbf{3}$ are the biotransformation products of fusaric acid by G. roseum under our growth conditions, while the origination of compound $\mathbf{1}$ and compound $\mathbf{4}$ are still not really clear. The isolation of new secondary metabolites from our fungal interaction cultures confirmed that the co-cultivation of two or more microbes is an efficient method for the discovery of new structures (Bertrand et al., 2013; Cueto et al., 2001; Park et al., 2009).

The biosynthetic pathway of compound $\mathbf{2}$ and compound $\mathbf{3}$ suggesting that biotransformation could be one of the responsible mechanisms for the production of 
fungal interaction secondary metabolites. Our bioassay noted that the compounds $\mathbf{1 - 3}$ are less toxic than fusaric acid, especially compound $\mathbf{2}$ is the least toxic, towards Botrytis cinerea and Aspergillus niger. This suggests that G. roseum could detoxify fusaric acid into other metabolites. Detoxification of fusaric acid by using different microorganisms have been carried out for several times (Utsumi et al., 1988; Fakhouri et al., 2003). Crutcher et al. (2014) recently reported that Aspergillus tubingensis can detoxify fusaric acid into fusarinol. In addition, G. roseum had been proved to possess the ability to transform nigranoic acid into three new products or hydroxylation of sesquiterpene into several derivatives (Dong et al., 2007; García-Granados et al., 2001 and 2002). Here we report for the first time that G. roseum was able to detoxify fusaric acid into two new fusaric acid derivatives 2 and 3. Notably, a hypothetical biosynthetic pathway for compound $\mathbf{2}$ could be formatted from amidation between FA produced from $F$. verticillioides and 4-aminobutyric acid derived from G. roseum. Compound 3 could be resulting from methyl esterification of compound 2. According to the significantly reduced antifungal activity of compound 2 when compared with fusaric acid, its structure-activity relationship supported the previous indication that the carboxylic acid in fusaric acid appeared to be necessary for the toxic activity (Crutcher et al., 2014).

Although fusaric acid could be degraded by G. roseum, the production of fusaric acid in the $G$. roseum and $F$. verticillioides co-cultures was not significantly affected by G. roseum. We assume that it is possible during the time when G. roseum detoxified fusaric acid, the fungus $F$. verticillioides kept producing fusaric acid simultaneously in the interaction cultures for surviving in this competitive environment. Accordingly, the four compounds are likely to originate from the fusaric acid-type precursors during the interaction. We emphasize that fusaric acid play an important role in the competitive interaction environments.

As a plant toxin, fusaric acid is associated with damping-off and root rot diseases of many vegetables and crops, to the mammals and brine shrimp fusaric acid is considered as a mild toxin (Capasso et al., 1996). Several approaches have been carried out previously to detoxified fusaric acid by using different microorganisms (Utsumi et al., 1988; Fakhouri et al., 2003). In this study, we point out G. flavor-fuscum and T. harzianum also could degrade fusaric acid under our culture conditions, while the toxicity of their biotransformation products should be tested for the further research to check whether they could be used as detoxification 
microorganism.

Efforts to obtain antagonistic microorganisms effective against plant pathogens have been underway in recent years. As a result, several microbes have demonstrated effectively as plant pathogen antagonists and some related biocontrol products are currently commercially available, including GleoGard (Trichoderma virens), Kodiak (Bacillus sp.), and BioTrek (Trichoderma sp.), TRICHODEX (T. harzianum), and BINAB-T (T. harzianum plus T. polysporum). The mechanism of microbial derived control agent has been characterized in four modes by which a bioagent can effectively against pathogen as including: a source of toxic antibiotic substances; a competitor for the survival food; a parasitic attack against pathogen, or an induced indirect toxic effect by the release of volatile compounds. Interestingly, our results display a different route on the biocontrol action. Firstly, the toxin fusaric acid was produced by the plant pathogen $F$. verticillioides, and then, the bioagent strain G. roseum transform fusaric acid into some lower toxic products through its own metabolic system. As such, G. roseum can be used as a biocontrol agent against F. verticillioides through a detoxification manner.

Briefly, the formation of the fusaric acid derivatives in the co-culture of F. verticillioides and G. roseum indicated that fusaric acid plays an important role in the interactions of these two fungi which could help us to understand the effect of the competition on their secondary metabolites.

\section{References}

Bacon, C.W., Hinton, D.M., 1996. Fusaric acid and pathogenic interactions of corn and non-corn isolates of Fusarium moniliforme, a nonobligate pathogen of corn. Adv. Exp. Med. Biol. 392,175-191.

Bertrand, S., Schumpp, O., Bohni, N., Monod, M., Gindro, K., Wolfender, J.L., 2013. De novo production of metabolites by fungal co-culture of Trichophyton rubrum and Bionectria ochroleuca. J. Nat. Prod. 76, 1157-1165.

Brandfass, C., Karlovsky, P., 2006. Simultaneous detection of Fusarium culmorum and $F$. graminearum in plant material by duplex PCR with melting curve analysis. BMC Microbiol. 6 (4), 1-10. 
Brown, D.W., Butchko, R.A.E., Busman, M., Proctor, R.H., 2012. Identification of gene clusters associated with fusaric acid, fusarin, and perithecial pigment production in Fusarium verticillioides. Fungal Genet. Biol. 49, 521-532.

Burmeister, H.R., Grove, M.D., Peterson, R.E., Weisleder, D., Plattner, R.D., 1985. Isolation and characterization of two new fusaric acid analogs from Fusarium moniliforme NRRL 13,163. Appl. Environ. Microbiol. 50, 311-314.

Capasso, R., Evidente, A., Cutignano, A., Vurro, M., Zonno, M.C., Bottalico, A., 1996. Fusaric and 9,10-dehydrofusaric acids and their methyl esters from Fusarium nygamai. Phytochemistry 41, 1035-1039.

Crutcher, F., Liu, J., Puckhaber, L., Stipanovic, R., Duke, S., Bell, A., Williams, H., Nichols, R., 2014. Conversion of fusaric acid to fusarinol by Aspergillus tubingensis: a detoxification reaction. J. Chem. Ecol. 40, 84-89.

Cueto, M., Jensen, P.R., Kauffman, C., Fenical, W., Lobkovsky, E., Clardy, J., 2001. Pestalone, a new antibiotic produced by a marine fungus in response to bacterial challenge. J. Nat. Prod. 64, 1444-1446.

Degenkolb, T., Heinze, S., Schlegel, B., Strobel, G., Grafe, U., 2002. Formation of new lipoaminopeptides, acremostatins $\mathrm{A}, \mathrm{B}$, and $\mathrm{C}$, by co-cultivation of Acremonium sp.Tbp-5 and Mycogone rosea DSM 12973. Biosci. Biotechnol. Biochem. 66 (4), 883-886.

Dong, J.Y., Chen, Y.G., Song, H.C., Zhu, Y.H., Zhou, Y.P., Li, L., He, Y.P., Cao, J., Zhang, K.Q., 2007. Hydroxylation of the triterpenoid nigranoic acid by the fungus Gliocladium roseum YMF1.00133. Chem. Biodivers. 4, 112-117.

Fakhouri, W., Walker, F., Armbruster, W., Buchenauer, H., 2003. Detoxification of fusaric acid by a nonpathogenic Colletotrichum sp. Physiol. Mol. Plant Pathol. 63, 263-269.

García-Granados, A., Gutiérrez, M.C., Rivas, F., Arias, J.M., 2001. Biotransformation of 4 $\beta$-hydroxyeudesmane-1,6-dione by Gliocladium roseum and Exserohilum halodes. Phytochemistry 58, 891-895.

García-Granados, A., Gutiérrez, M.C., Parra, A., Rivas, F., 2002. Chemical-microbiological synthesis of cryptomeridiol derivatives by 
Gliocladium roseum: semisynthesis of 11-hydroxyeudesmanolides. J. Nat. Prod. 65, 1011-1015.

Jensen, B., Knudsen, I.B., Jensen, D., 2000. Biological seed treatment of cereals with fresh and long-term stored formulations of Clonostachys rosea: biocontrol efficacy against Fusarium culmorum. Eur. J. Plant Pathol. 106, 233-242.

Li, C., Zhang, J., Shao, C., Ding, W., She, Z., Lin, Y., 2011. A new xanthone derivative from the co-culture broth of two marine fungi (strain No. E33 and K38). Chem. Nat. Compd. 47, 382-384.

Li, G.Q., Huang, H.C., Kokko, E.G., Acharya, S.N., 2002. Ultrastructural study of mycoparasitism of Gliocladium roseum on Botrytis cinerea. Bot. Bull. Acad .Sin. 43, 211-218.

Marasas, W.F.O., Riley, R.T., Hendricks, K.A., Stevens, V.L., Sadler, T.W., Gelineau-van Waes, J., Missmer, S.A., Cabrera, J., Torres, O., Gelderblom, W.C.A., Allegood, J., Martínez, C., Maddox, J., Miller, J.D., Starr, L., Sullards, M.C., Roman, A.V., Voss, K.A., Wang, E., Merrill, A.H., 2004. Fumonisins disrupt sphingolipid metabolism, folate transport, and neural tube development in embryo culture and in vivo: a potential risk factor for human neural tube defects among populations consuming fumonisin-contaminated maize. J. Nutr. $134,711-716$.

Misra, R.N., Karanewsky, S., 1985. 2-pyridylcarboxamides. CA1264753 A1.

Mukherjee, P.K., Nautiyal, C.S., Mukhopadhyay, A.N., 2008. Molecular mechanisms of biocontrol by Trichoderma spp. in: Nautiyal, C., Dion, P. (Eds.), Molecular Mechanisms of Plant and Microbe Coexistence, Soil Biology, pp: 243-262.

Mulè, G., Susca, A., Stea, G., Moretti, A., 2004. A species-specific PCR assay based on the calmodulin partial gene for identification of Fusarium verticillioides, $F$. proliferatum and F. subglutinans. Eur. J. Plant Pathol. 110, 495-502.

Nutz, S., Döll, K., Karlovsky, P., 2011. Determination of the LOQ in real-time PCR by receiver operating characteristic curve analysis: application to qPCR assays for Fusarium verticillioides and F. proliferatum. Anal. Bioanal. Chem. 401, $717-726$. 
Oh, D.C., Kauffman, C.A., Jensen, P.R., Fenical, W., 2007. Induced production of emericellamides A and B from the marine-derived fungus Emericella sp. in competing co-culture. J. Nat. Prod. 70, 515-520.

Onaka, H., Mori, Y., Igarashi, Y., Furumai, T., 2011. Mycolic acid-containing bacteria induce natural-product biosynthesis in Streptomyces species. Appl. Environ. Microbiol. 77, 400-406.

Pan, J.H., Lin, Y.C., Tan, N., Gu, Y.C., 2010. Cu (II): A 'singaling molecule' of the mangrove endophyte Fusarium oxysporum ZZF51? Biometals 23, 1053-1060.

Park, H.B., Kwon, H.C., Lee, C.H., Yang, H.O., 2009. Glionitrin A, an antibiotic-antitumor metabolite derived from competitive interaction between abandoned mine microbes. J. Nat. Prod. 72, 248-252.

Pettit, R., 2009. Mixed fermentation for natural product drug discovery. Appl. Microbiol. Biotechnol. 83, 19-25.

Presello, D.A., Botta, G., Iglesias, J., Eyhérabide, G.H., 2008. Effect of disease severity on yield and grain fumonisin concentration of maize hybrids inoculated with Fusarium verticillioides. Crop Prot. 27, 572-576.

Ratzinger, A., Riediger, N., von Tiedemann, A., Karlovsky, P., 2009. Salicylic acid and salicylic acid glucoside in xylem sap of Brassica napus infected with Verticillium longisporum. J. Plant Research 122, 571-579.

Renslo, A.R., Danheiser, R.L., 1998. Synthesis of substituted pyridines via regiocontrolled $[4+2]$ cycloadditions of oximinosulfonates. J. Org. Chem. 63, $7840-7850$.

Ross, P.F., Nelson, P.E., Richard, J.L., Osweiler, G.D., Rice, L.G., Plattner, R.D., Wilson, T.M., 1990. Production of fumonisins by Fusarium moniliforme and Fusarium proliferatum isolates associated with equine leukoencephalomalacia and a pulmonary edema syndrome in swine. Appl. Environ. Microbiol. 56, 3225-3226.

Sanchez-Rangel, D., SanJuan-Badillo, A., Plasencia, J., 2005. Fumonisin production by Fusarium verticillioides strains isolated from maize in Mexico and development of a polymerase chain reaction to detect potential toxigenic 
strains in grains. J. Agric. Food Chem. 53, 8565-8571.

Scherlach, K., Hertweck, C., 2009. Triggering cryptic natural product biosynthesis in microorganisms. Org. Biomol. Chem. 7, 1753-1760.

Schroeckh, V., Scherlach, K., Nützmann, H.W., Shelest, E., Schmidt-Heck, W., Schuemann, J., Martin, K., Hertweck, C., Brakhage, A.A., 2009. Intimate bacterial-fungal interaction triggers biosynthesis of archetypal polyketides in Aspergillus nidulans. Proc. Natl. Acad. Sci. 106, 14558-14563.

Slattery, M., Rajbhandari, I., Wesson, K., 2001. Competition-mediated antibiotic induction in the marine bacterium Streptomyces tenjimariensis. Microb. Ecol. 41, 90-96.

Sutton, J.C., Li, D.W., Peng, G., Yu, H., Zhang, P., 1997. Gliocladium roseum, a versatile adversary of Botrytis cinerea in crops. Plant Dis. 81, 316-328.

Tan, N., Pan, J.H., Peng, G.T., Mou, C.B., Tao, Y.W., She, Z.G., Yang, Z.L., Zhou, S.N., Lin, Y.C., 2008. A copper coordination compound produced by a marine fungus Fusarium sp. ZZF51 with biosorption of Cu (II) ions. Chin. J. Chem. $26,516-521$.

Tschesche, R., Führer, W., 1978. Eine neue synthese der 5-butyl-2-pyridincarbonsäure (fusarsäure) über die „Emmert-Reaktion”. Chem. Ber. 111, 3502-3505.

Utermark, J., Karlovsky, P., 2007. Role of zearalenone lactonase in protection of Gliocladium roseum from fungitoxic effects of the mycotoxin zearalenone. Appl. Environ. Microbiol. 73, 637-642.

Utsumi, R., Hadama, T., Noda, M., Toyoda, H., Hashimoto, H., Ohuchi, S., 1988. Cloning of fusaric acid-detoxifying gene from Cladosporium werneckii: a new strategy for the prevention of plant diseases. J. Biotechnol. 8, 311-316.

Xue, A.G., 2002. Gliocladium roseum strains useful for the control of fungal pathogens in plants. WO 2000018241 A1 20000406.

Yabuta, T., Kambe, K., Hayashi, T., 1934. Biochemistry of the bakanae-fungus, I. fusarinic acid, a new product of the bakanae-fungus. J. Agric. Chem. Soc. Jpn. 1059-1068.

Zhang, L., Yang, J., Niu, Q., Zhao, X., Ye, F., Liang, L., Zhang, K.Q., 2008. 
Investigation on the infection mechanism of the fungus Clonostachys rosea against nematodes using the green fluorescent protein. Appl. Microbiol. Biotechnol. 78, 983-990.

Zuck, K.M., Shipley, S., Newman, D.J., 2011. Induced production of N-Formyl alkaloids from Aspergillus fumigatus by co-culture with Streptomyces peucetius. J. Nat. Prod. 74, 1653-1657.

\section{Support information}

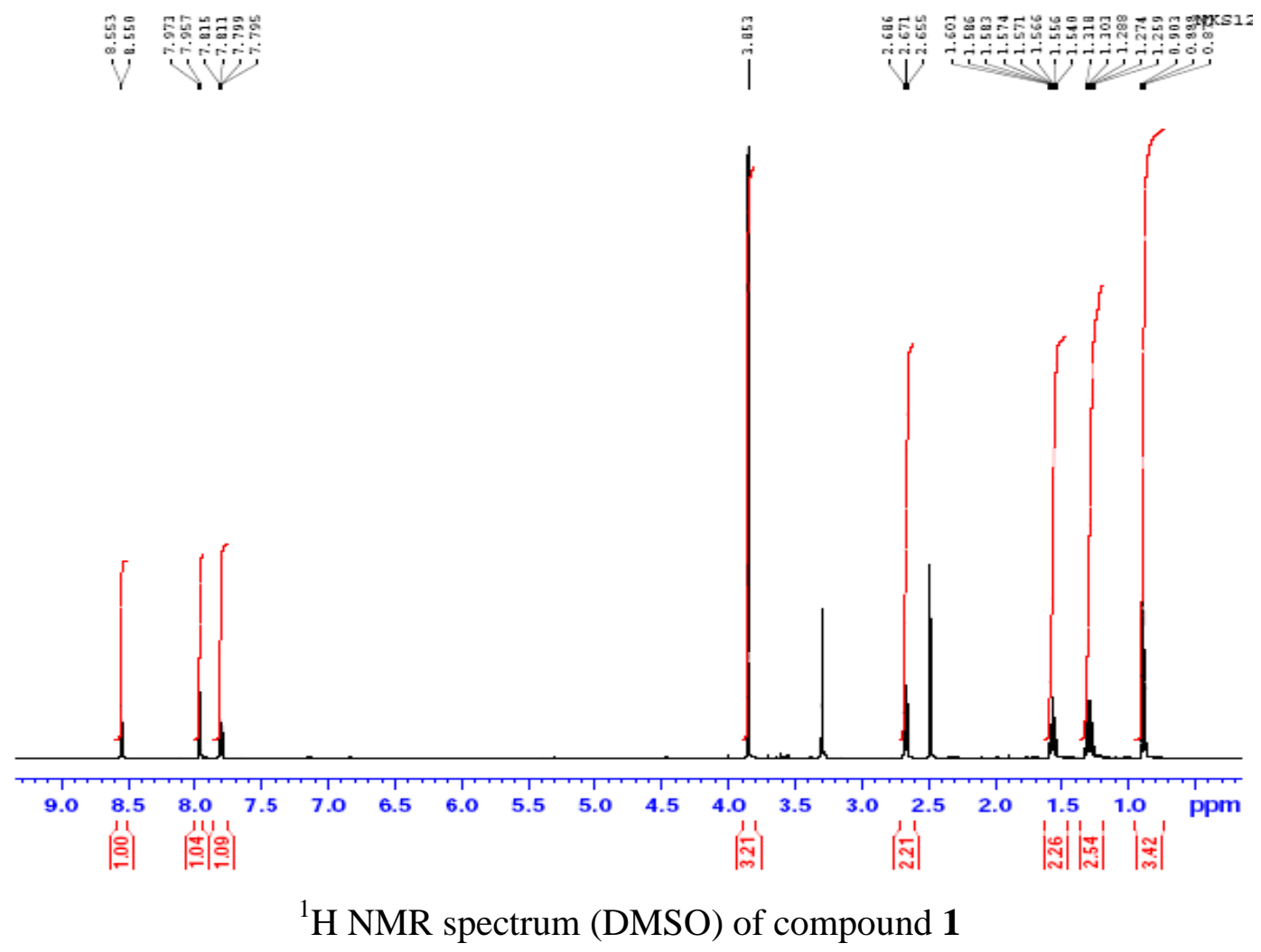




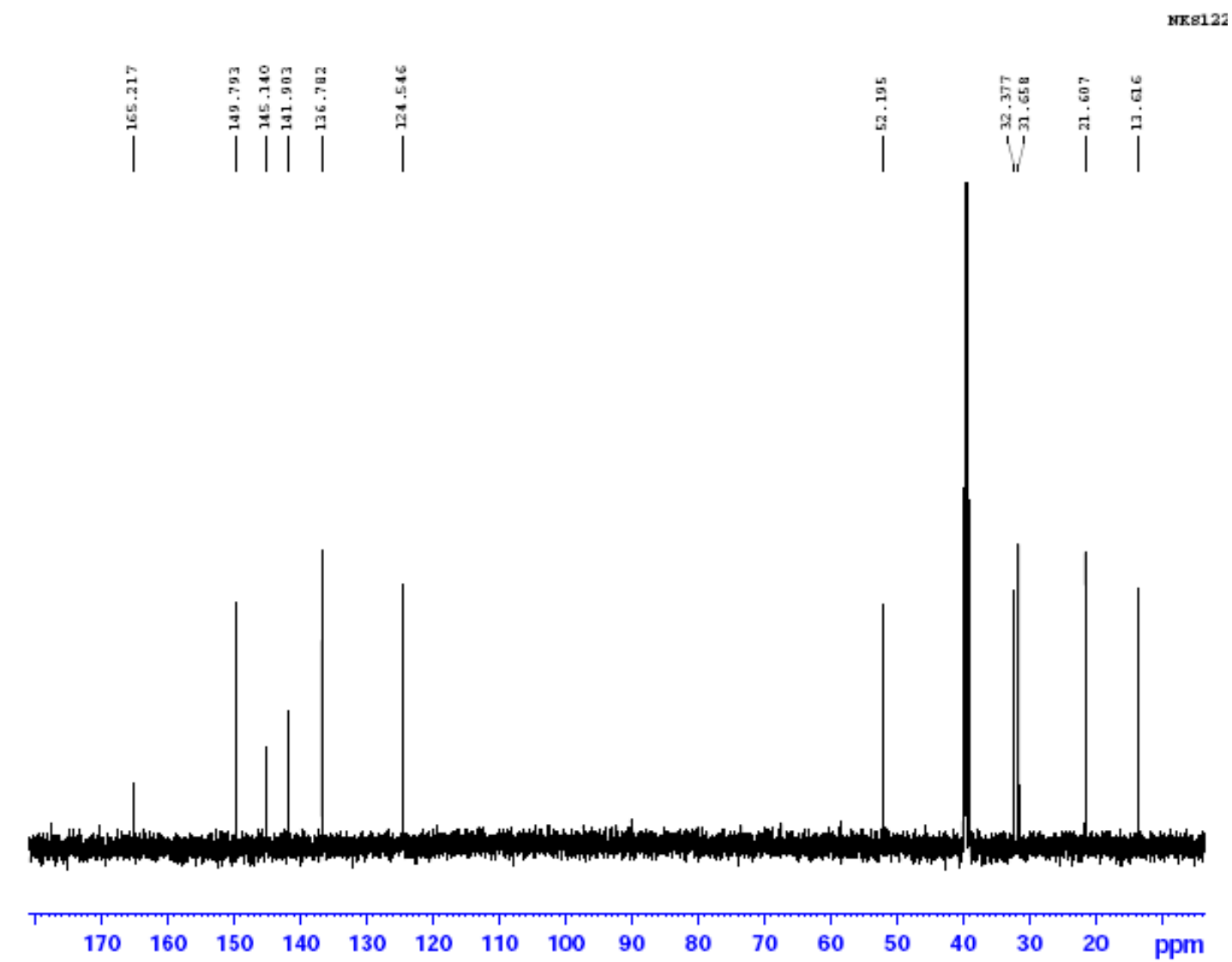

${ }^{13} \mathrm{C}$ NMR spectrum (DMSO) of compound $\mathbf{1}$

arasa/cks

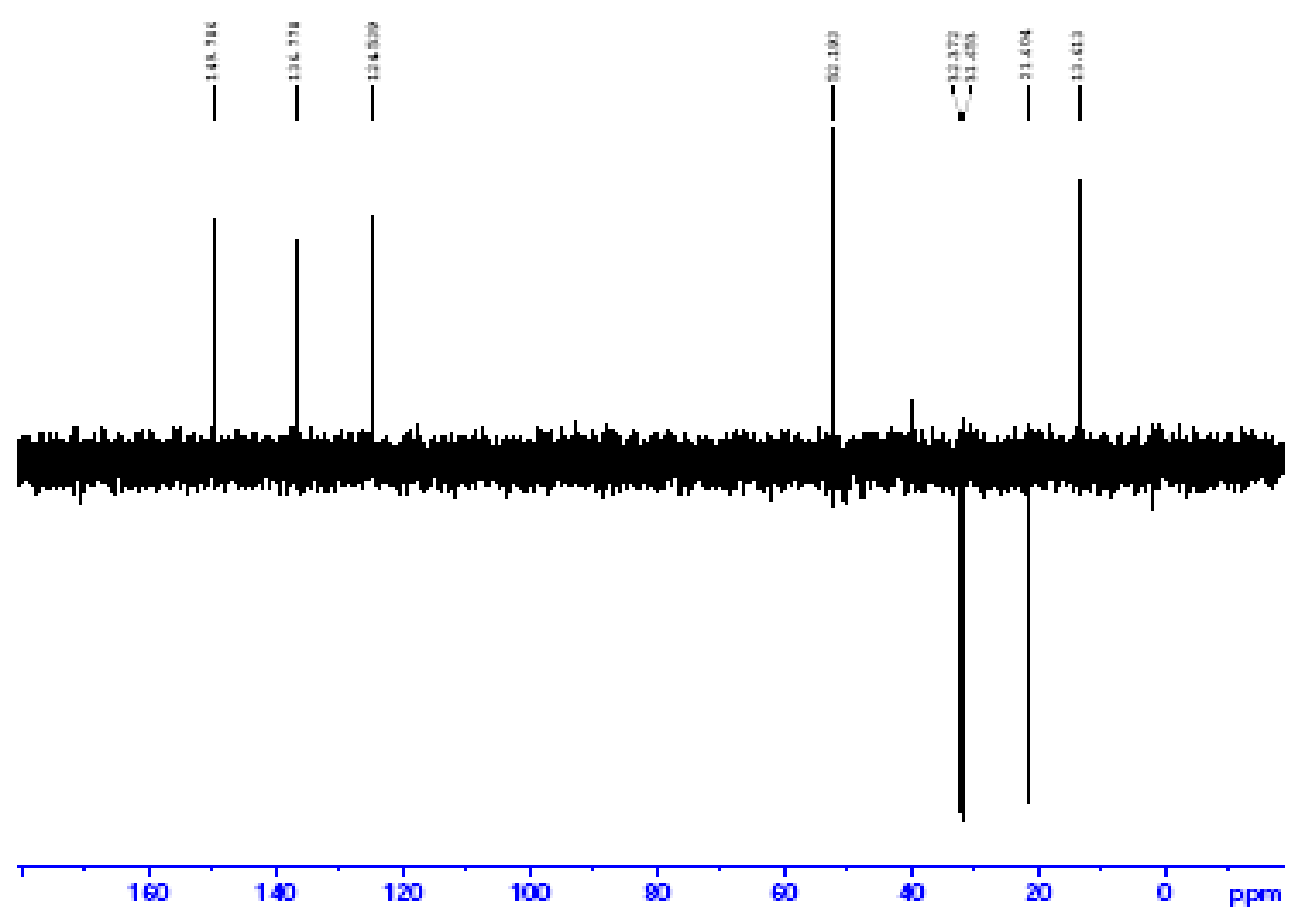

DEPT135 spectrum (DMSO) of compound 1 


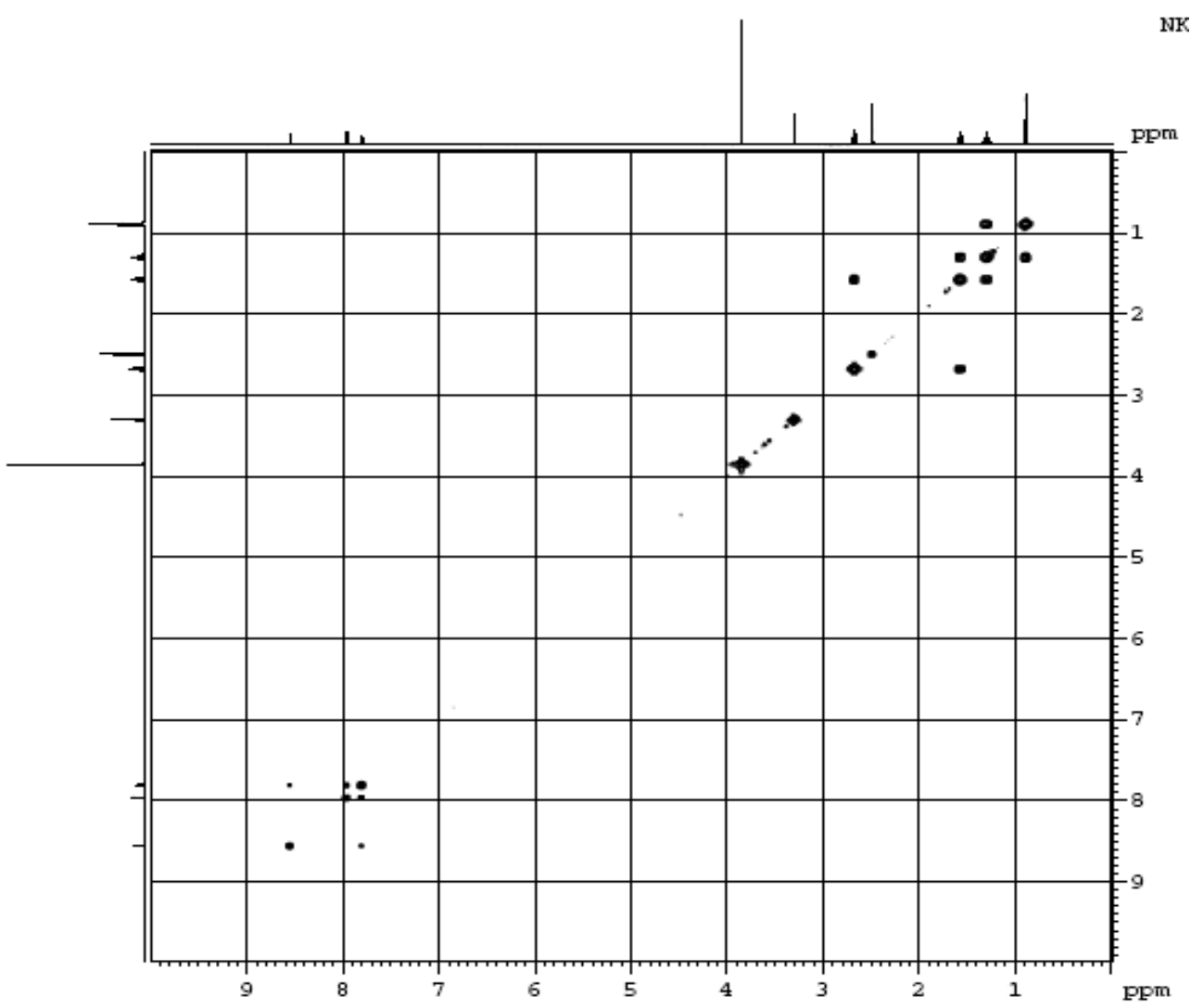

H, H COSY spectrum (DMSO) of compound $\mathbf{1}$

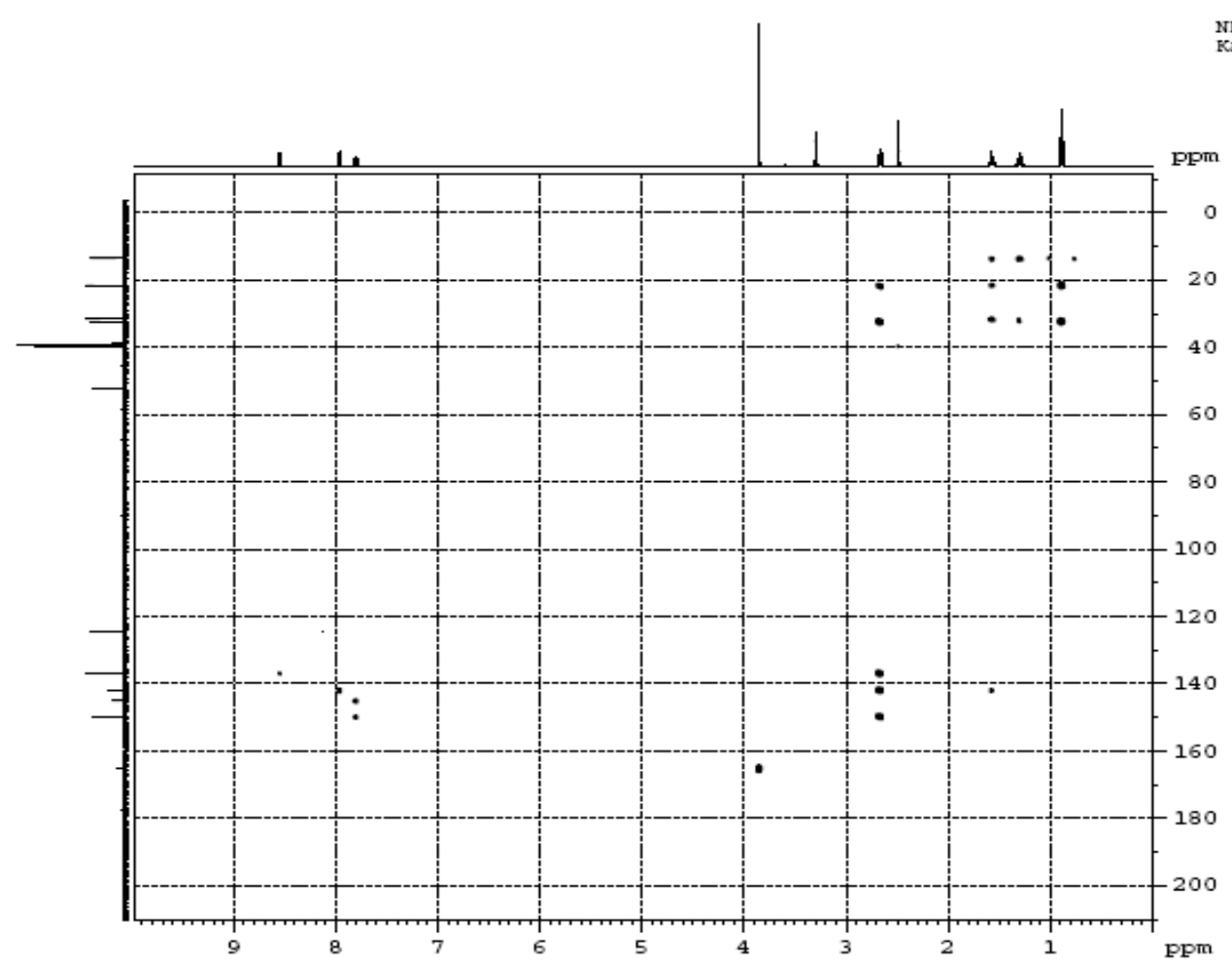

HMBC spectrum (DMSO) of compound 1 


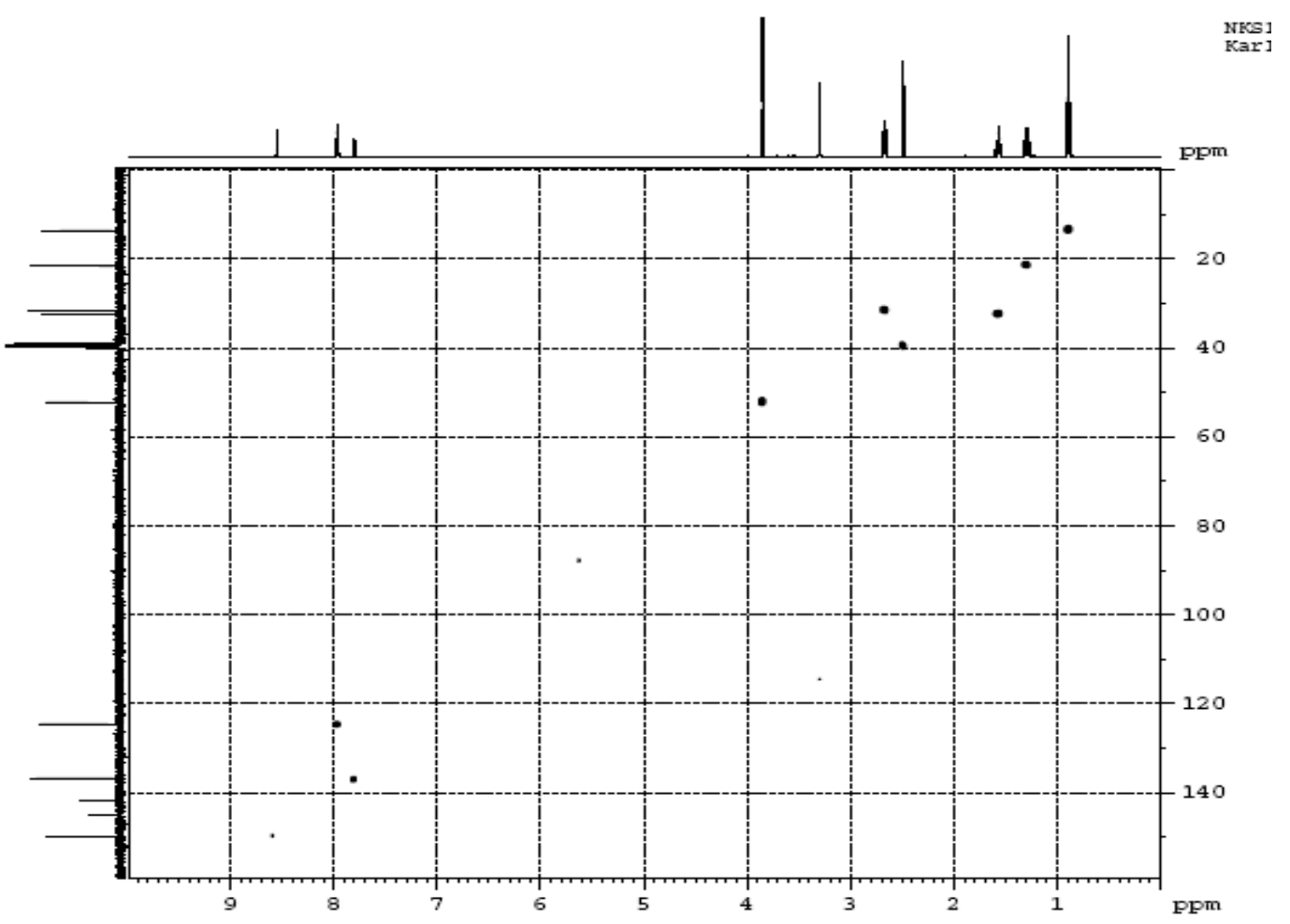

HSQC spectrum (DMSO) of compound 1

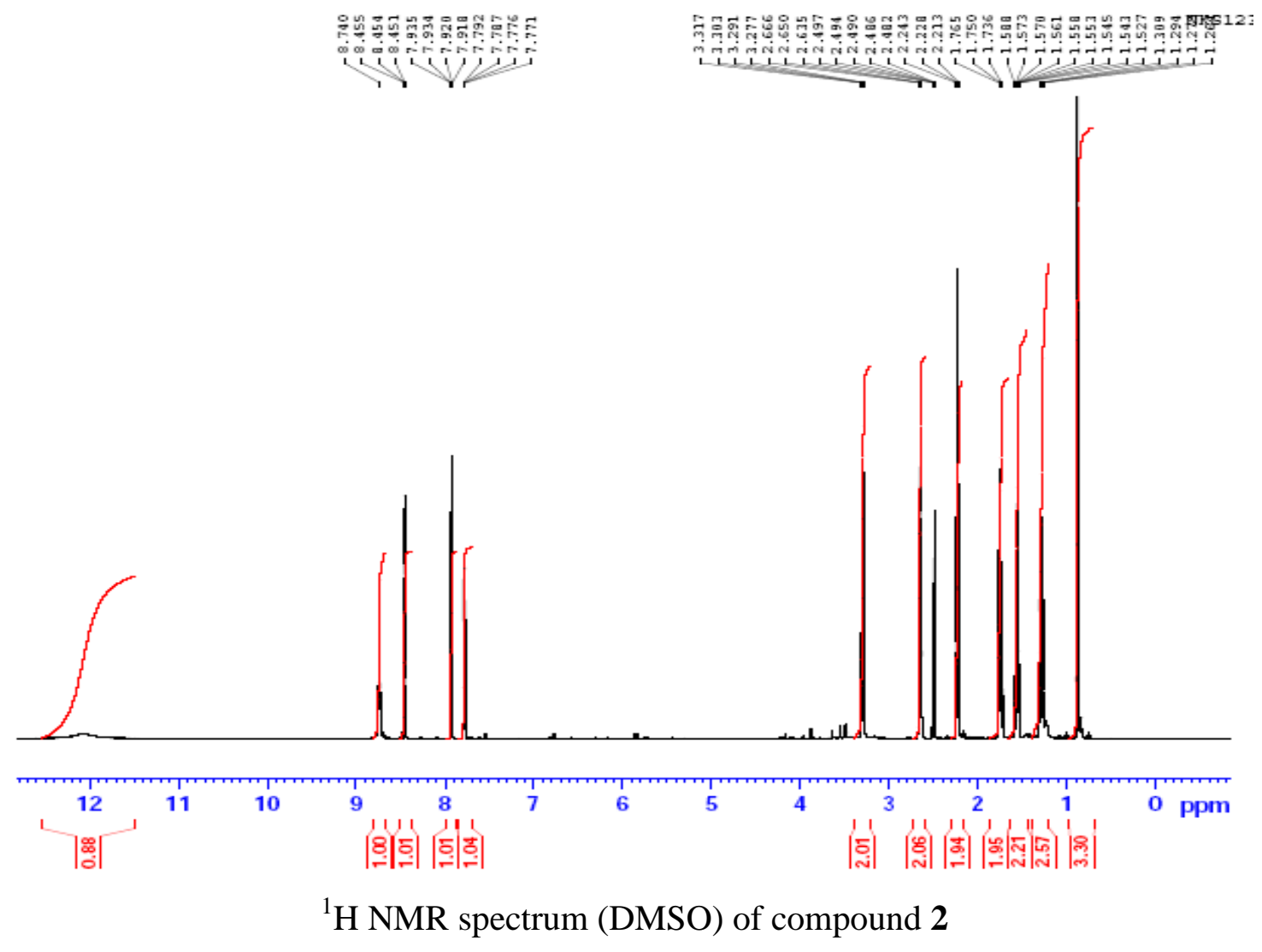



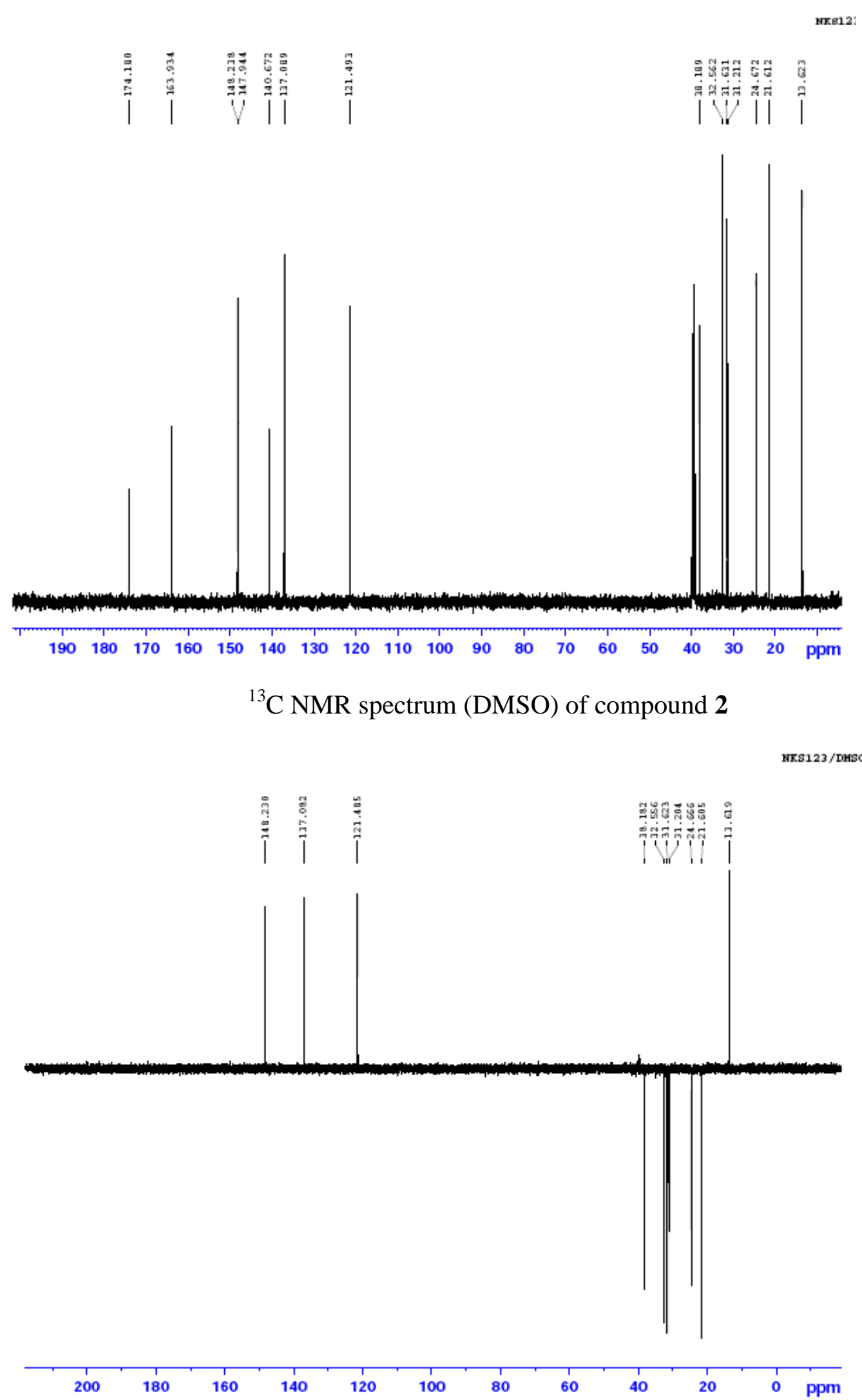

DEPT135 spectrum (DMSO) of compound 2 


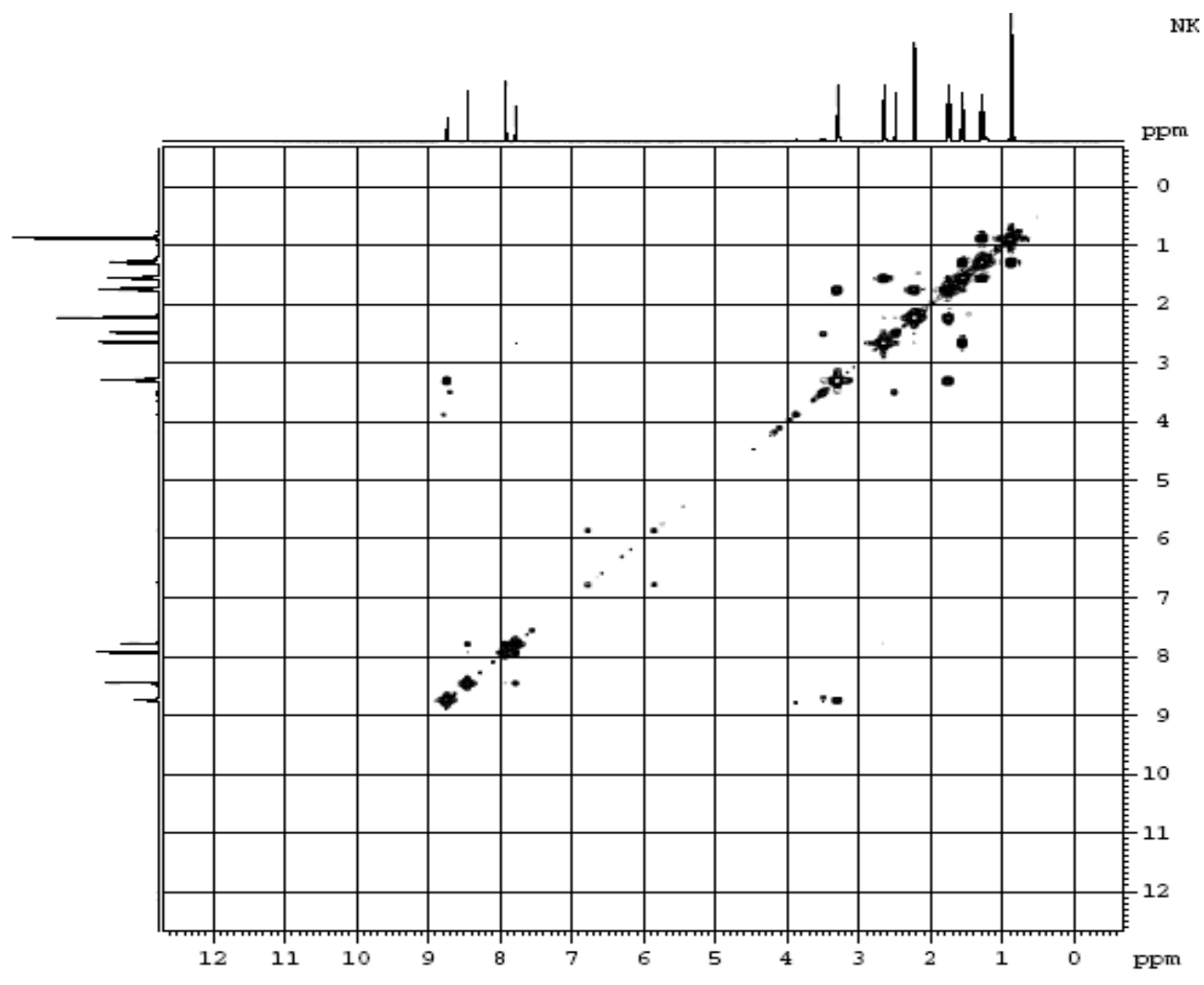

H, H COSY spectrum (DMSO) of compound 2

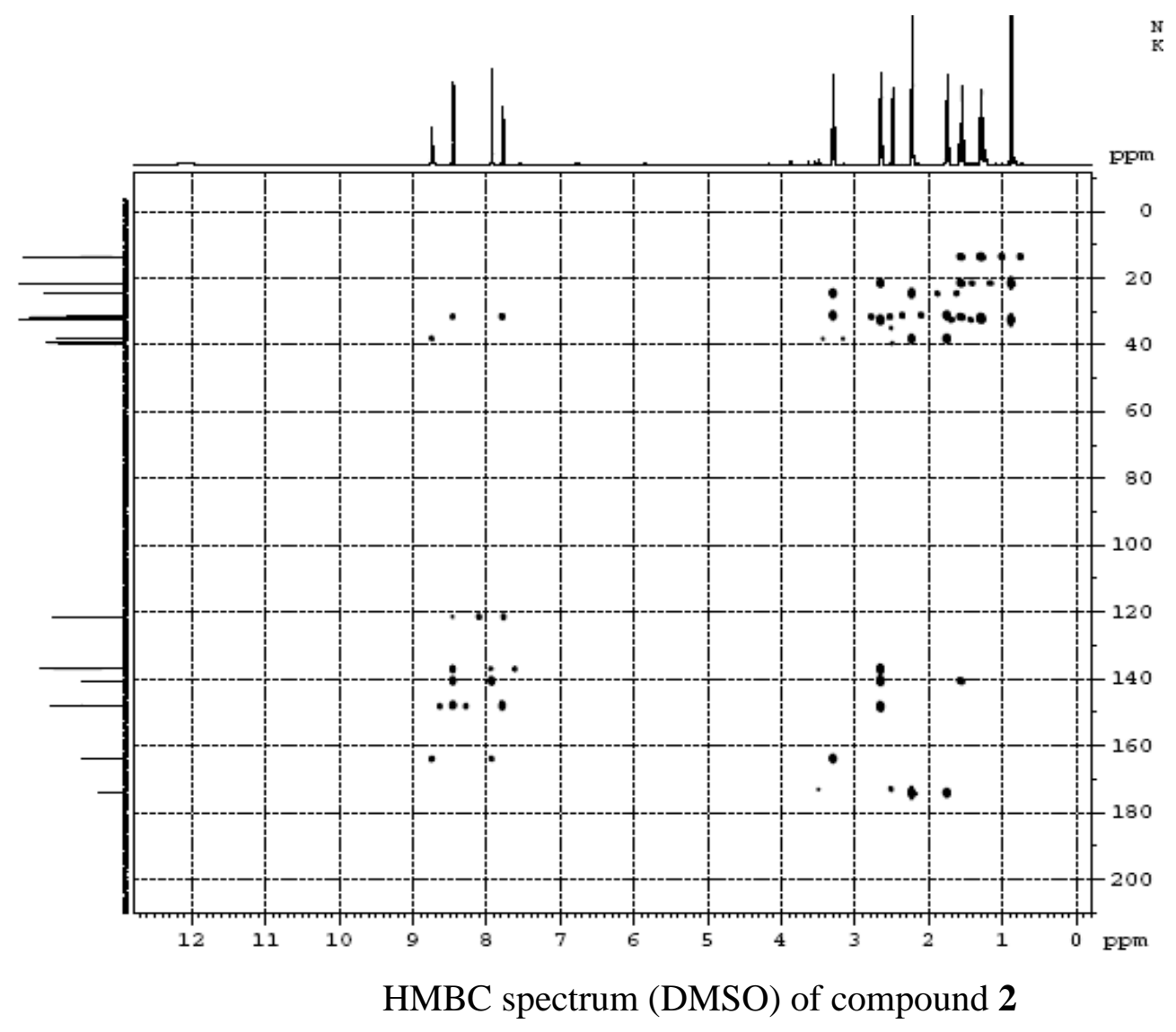




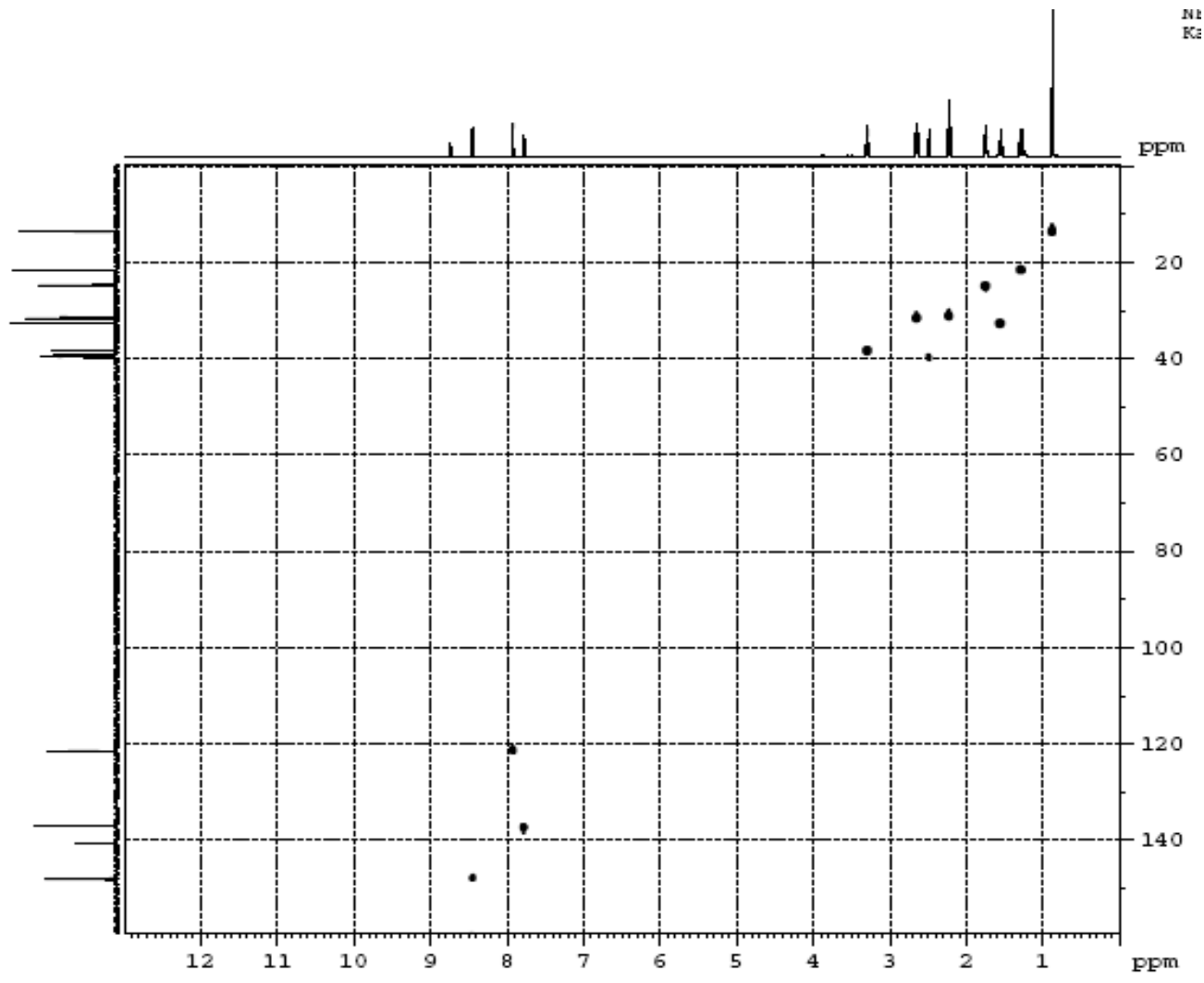

HSQC spectrum (DMSO) of compound 2

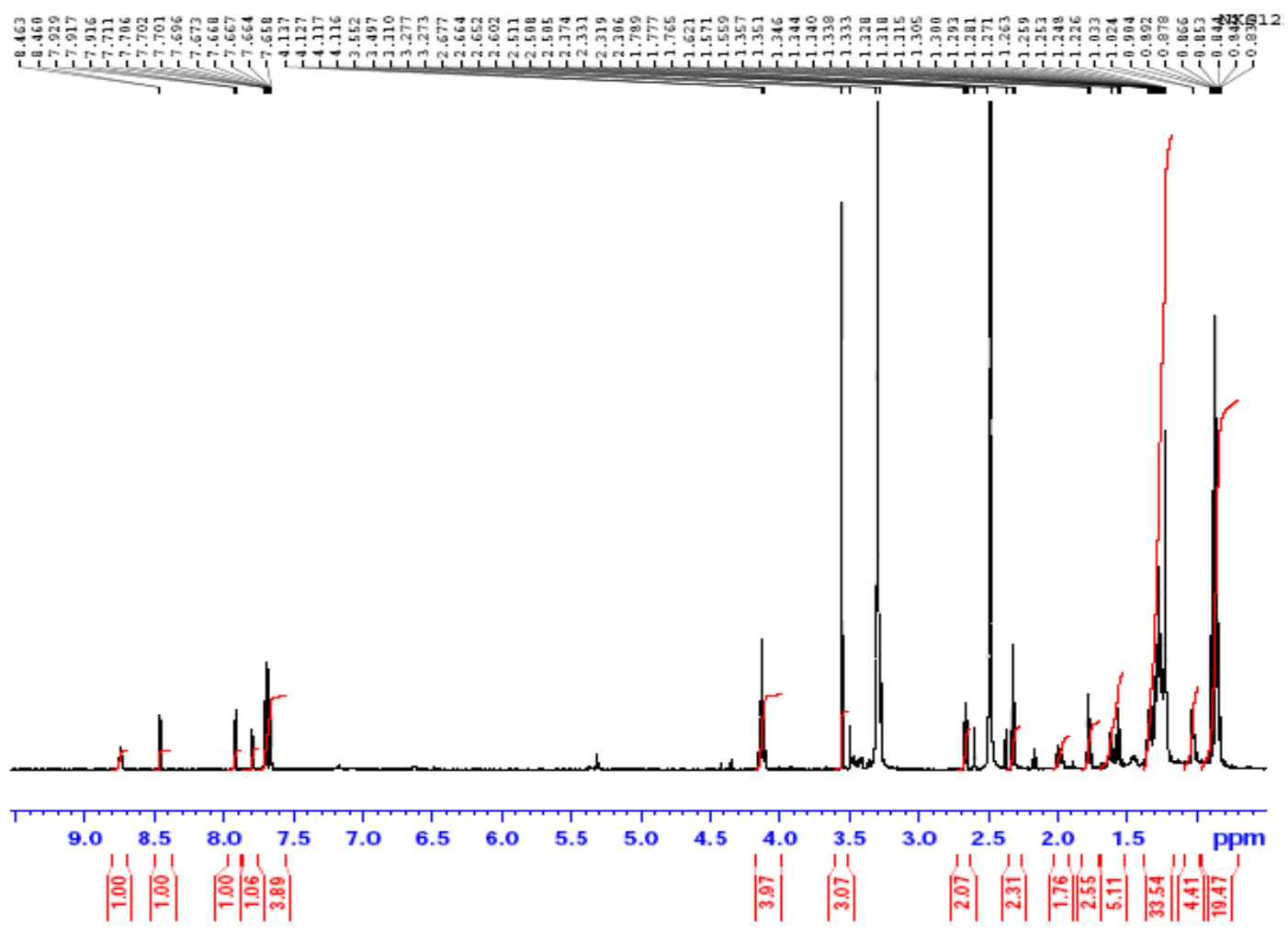

${ }^{1} \mathrm{H}$ NMR spectrum (DMSO) of compound $\mathbf{3}$ 

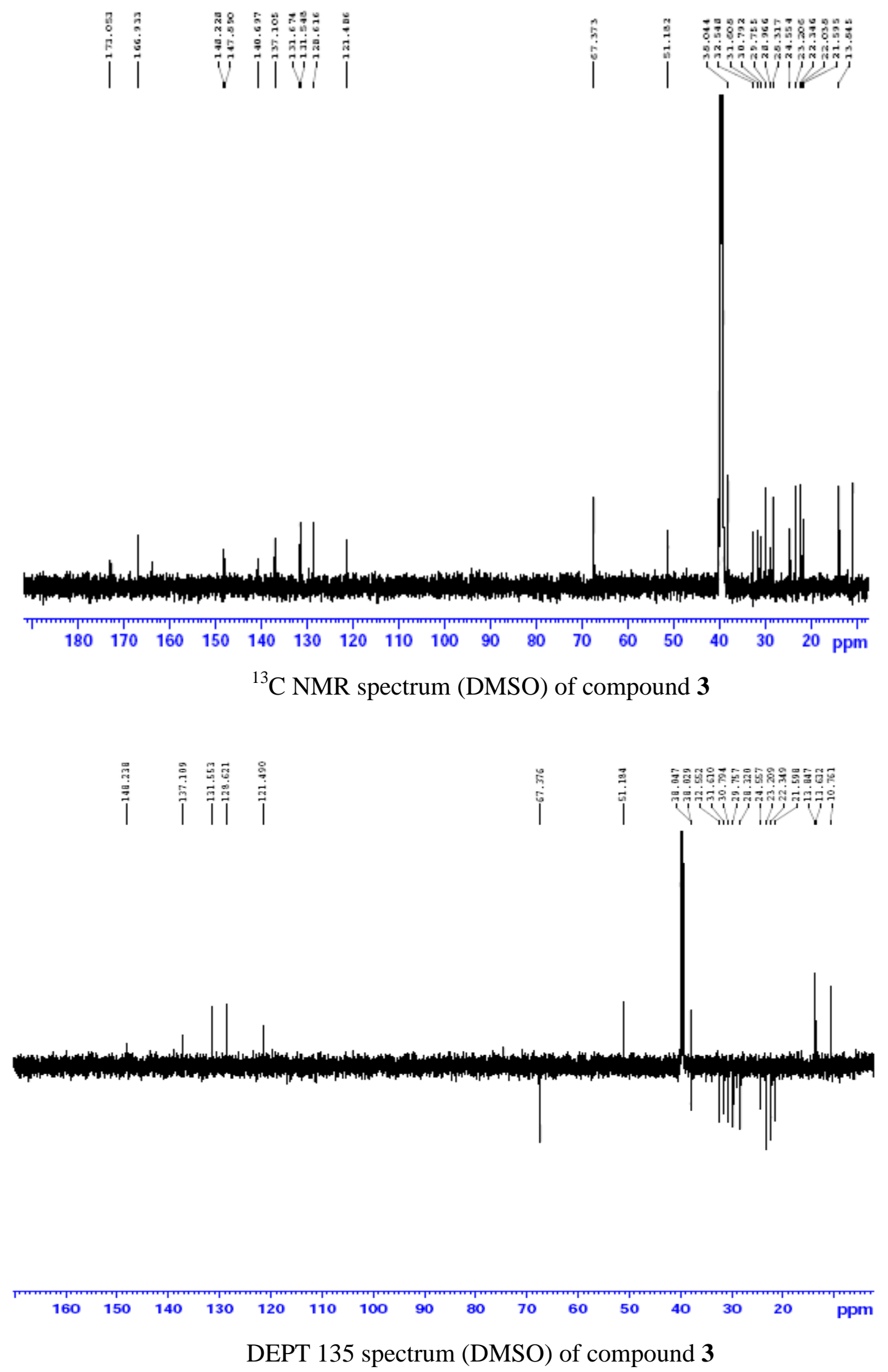


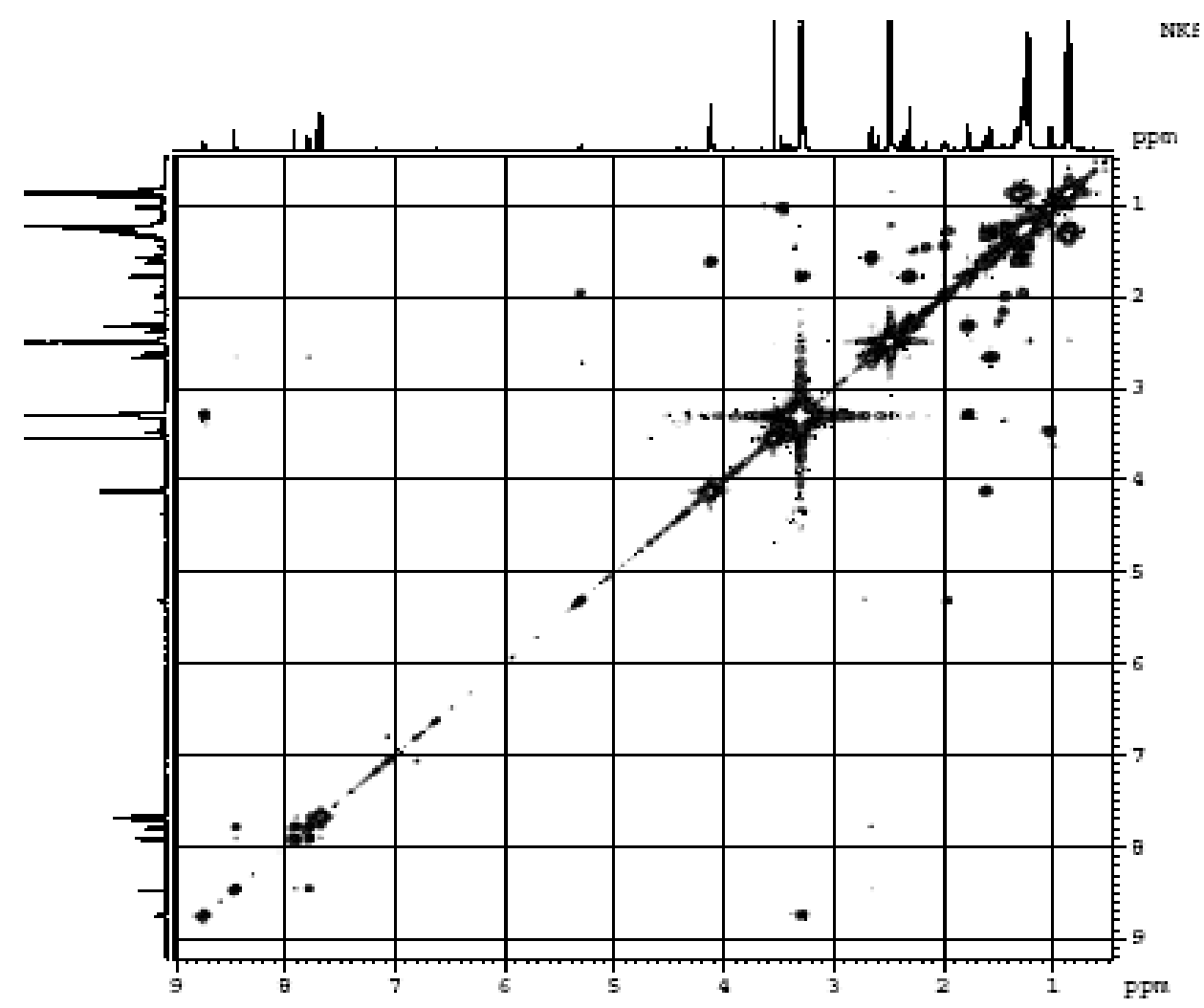

H, H COSY spectrum (DMSO) of compound 3

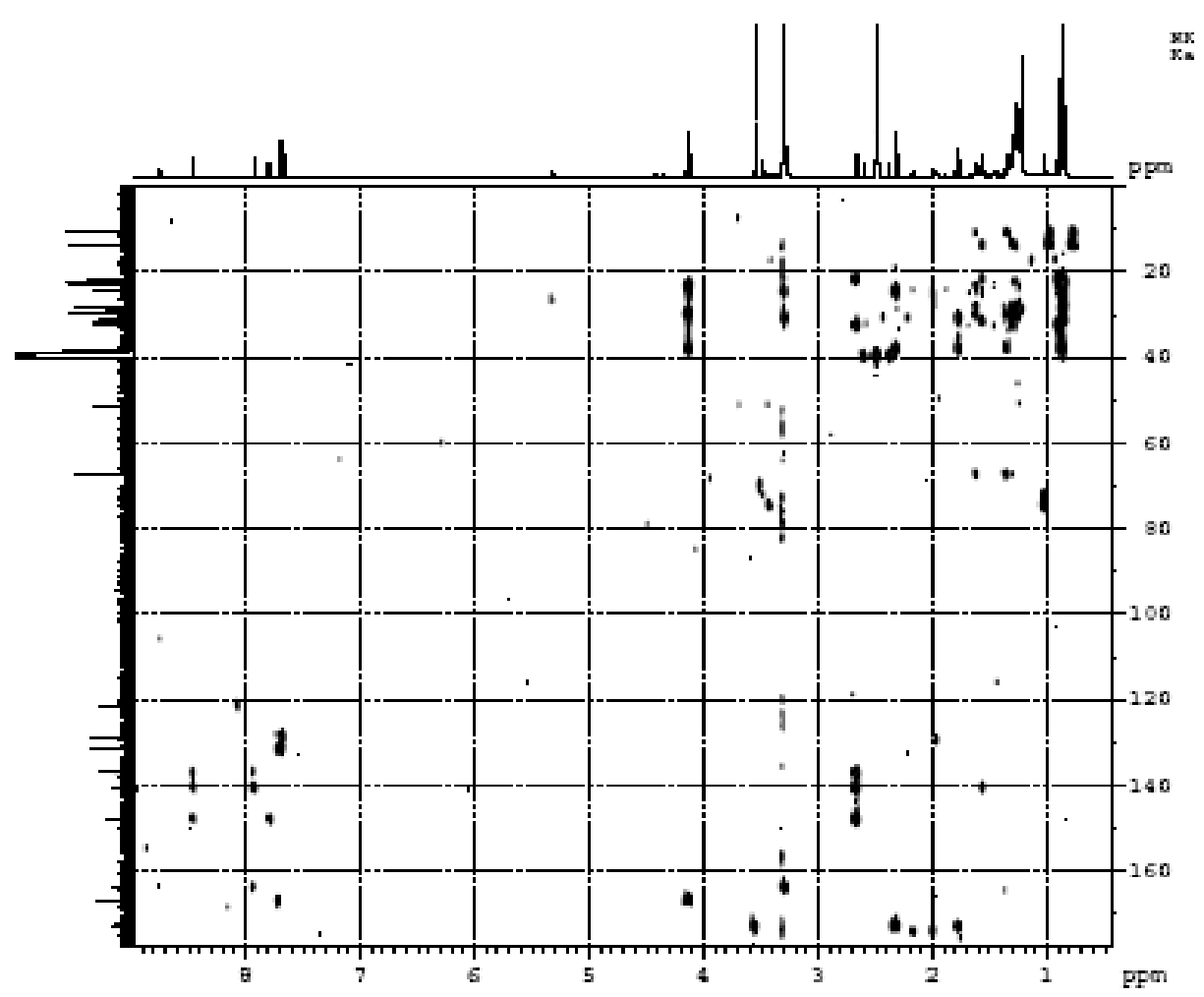

HMBC spectrum (DMSO) of compound 3 


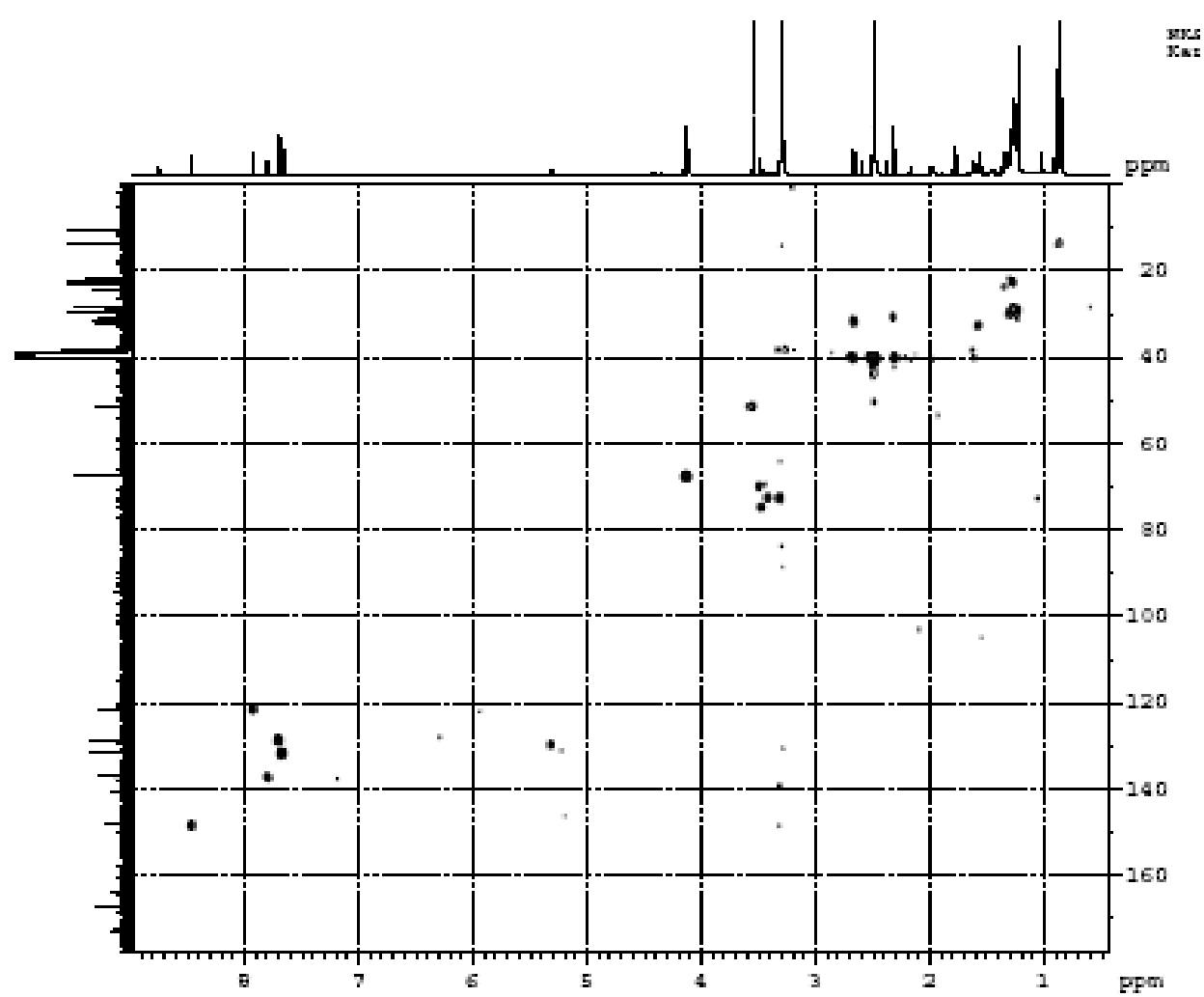

HSQC spectrum (DMSO) of compound $\mathbf{3}$

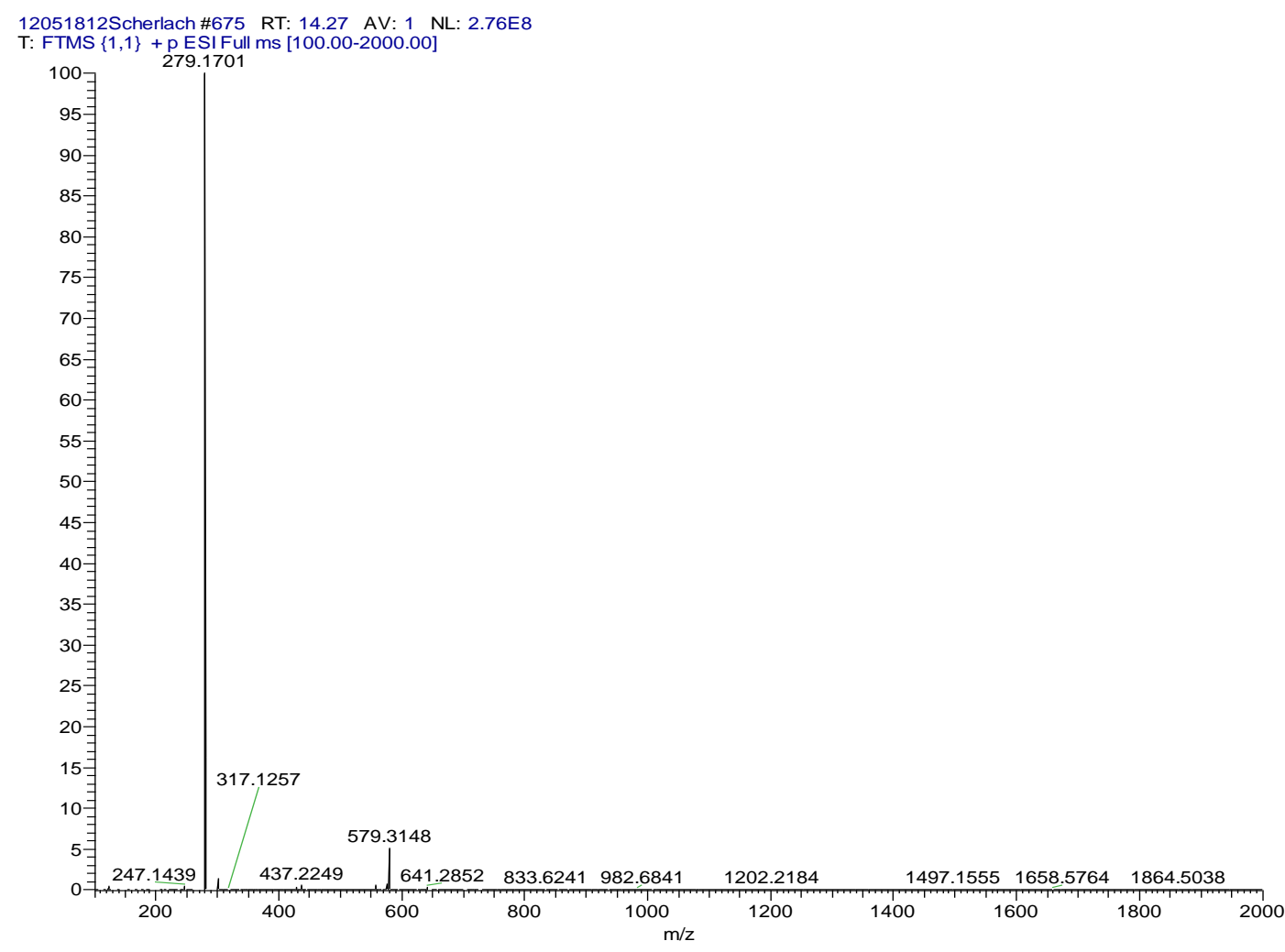

HRESIMS spectrum of compound $\mathbf{3}$ 


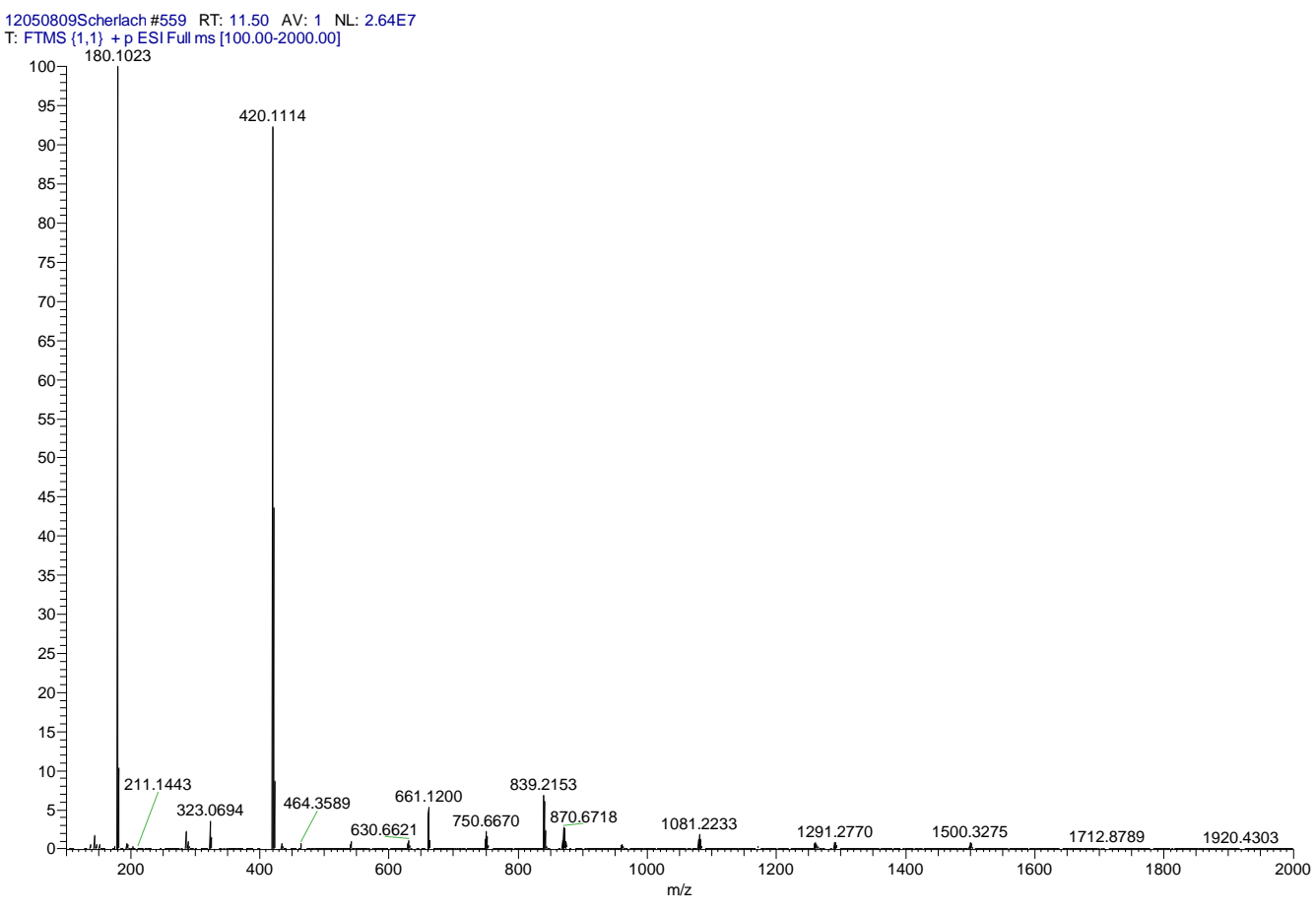

HRESIMS spectrum of compound 4

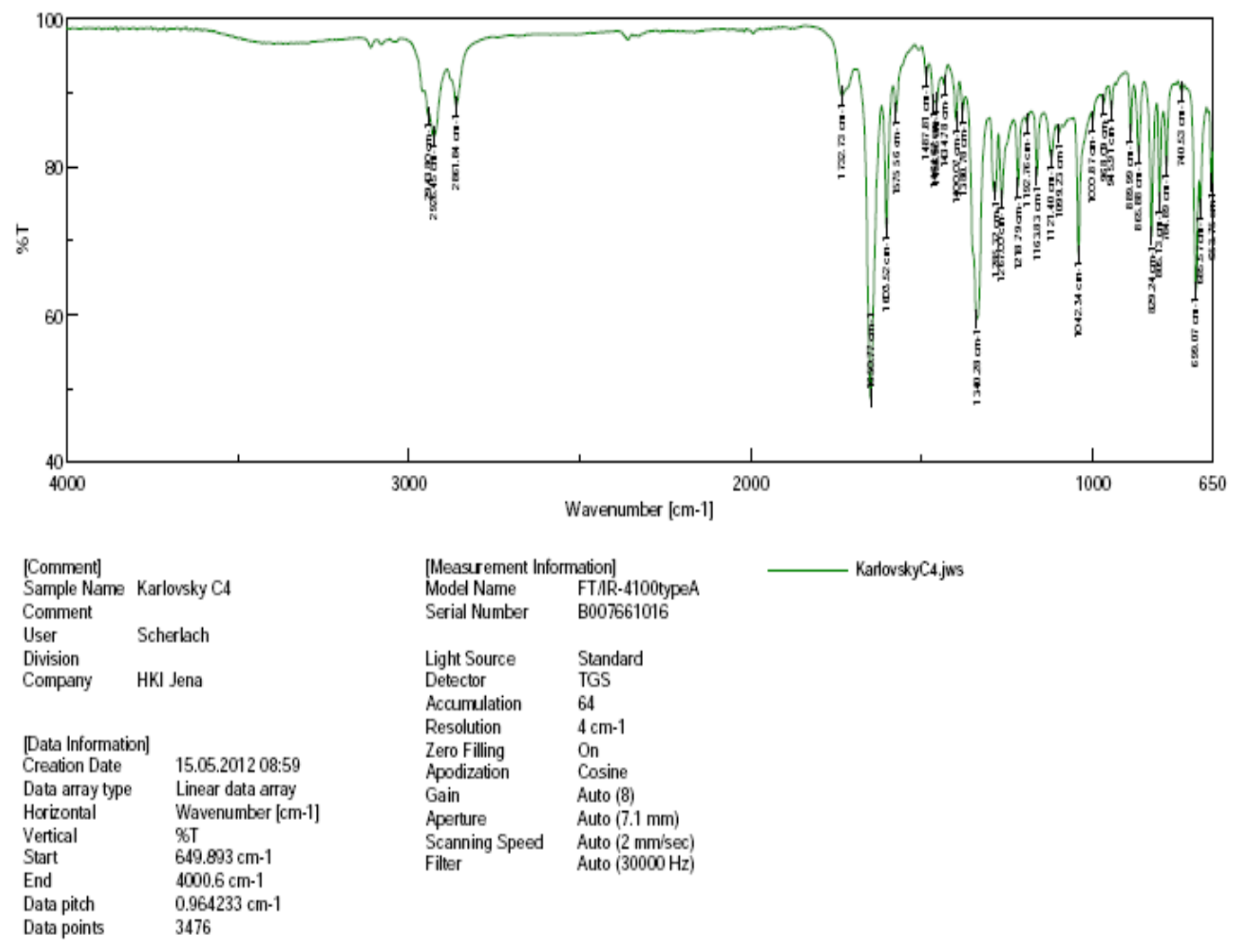

IR spectrum of compound 4 


\title{
Chapter 4: LC-MS based metabolic profiling of Botrytis
}

\section{species}

\author{
Yi Kuang, Franz Hadacek, Matthias Hahn, Petr Karlovsky*
}

\begin{abstract}
In this study, the secondary metabolites produced by 10 different Botrytis strains (Botrytis cinerea B05.10 and three mutants: B05.10pks6, B05.10bot2 and B05.10pks6bot2, Botrytis cinerea SAS405, Botrytis pseudocinerea VD110, Botrytis pseudocinerea VD184, Botrytis fabae 2240, Botrytis group Sa D08_H_8 and Botrytis group Sa G09_S33) were investigated. A HPLC-MS based non-targeted metabolic profiling was performed to identify their variation in secondary metabolites in different species and the changes in metabolic profiles caused by disruption of selected genes of secondary metabolism synthesis. Multivariate statistical analysis hierarchical clustering analysis (HCA) and non-metric multidimensional scaling (MDS) were used for chemotaxonomic classification. Most strains were well classified based on their secondary metabolites, the Botrytis group Sa was proved to be closely related to Botrytis cinerea. Changes in the metabolite production by gene disruption were found and some of the botrydial-related signals were characterized. The correlation with botrydial-related metabolites and fungal biomass suggest that the phytotoxin botrydial might cause effect in saprophytic growth.
\end{abstract}

\section{Introduction}

Botrytis cinerea Pers.:Fr. [teleomorph Botryotinia fuckeliana (de Bary) Whetzel] is a necrotrophic plant pathogenic fungus that grows as a gray mold in more than 200 commercial crops worldwide, including grapes, tomatoes and strawberries causing 
serious economic losses (Washington, 1993). It is considered to be one of the most dangerous plant pathogens (Dean et al., 2012). As a major species of the genus Botrytis, B. cinerea is a well-known producer of many structurally diverse metabolites, some of them showing phytotoxic activity, such as some polyketide lactones known as botrylactones (Welmar et al., 1979) and botcinolides (Jacyno et al., 1994), the best known being botrydial, dihydrobotrydial and their derivatives, which cause the typical lesions of fungal infections (Fehlhaber et al., 1974). The biosynthesis gene clusters of botrydial have been characterized previously, comprising five genes BcBOT1 to BcBOT5. BcBOT2 is a sesquiterpene cyclase which catalyzes the first step of botrydial synthesis (Pinedo et al., 2008). BcPKS6 and BcPKS9 have been identified as polyketide synthases (PKS) encoding genes, which are key enzymes for the biosynthesis of botcinic acid (Dalmais et al., 2011). Botrytis fabae Sard. is a necrotrophic pathogen which causes chocolate spot diseases and reduces the commercial production of faba bean (Vicia faba L.) worldwide (Torres et al., 2006). B. fabae kills the host cells probably by production of phytotoxins and enzymes when grows inside the plant (Harrison, 1983). Botrytone, which is produced by B. fabae, showed moderate phytotoxic activity to $V$. faba (Cimmino et al., 2011). B. pseudocinerea is a new cryptic species that was described first from isolates in French vineyards which is naturally resistant (phenotype HydR1) to the hydroxyanilide fungicide fenhexamid (Debieu et al., 2013). On grapevine, B. cinerea is the predominant species, in particular on ripe berries, while B. pseudocinerea was mainly detected during spring (Walker et al., 2011). A new genetic variant of B. cinerea, called Botrytis group Sa, was found to be widespread in German strawberry fields but almost absent from vineyards. Multiple-gene sequencing confirmed that Botrytis group Sa is closely related to B. cinerea and B. fabae (Leroch et al., 2013).

Metabolic profiling which used as an analytical approach to characterize the different biosynthetic capacity of an organism or group of organisms. The use of HPLC and GC coupled with mass spectrometry has been proven to be a powerful and popular tool for such chemical composition analysis (Fischedick et al., 2010; Heinrich et al., 
2013). Identification of unknown metabolites is possible by using $\mathrm{LC}^{-M S^{\mathrm{n}}}$ based metabolic profiling. In particular, non-targeted metabolic profiling could detect the metabolic differences which exist between species or caused by gene transfer (Hanhineva et al., 2009). The metabolomics approaches utilize multivariate statistical analyses of the metabolite profiles, which were shown to be an effective tool for the classification of microorganisms (Kang et al., 2011; Lee et al., 2013; Kim et al., 2009).

In this study, non-targeted metabolic profiling was used to (i) explore variation in secondary metabolites produced by isolates of Botrytis spp. specialized to different host plants and (ii) identify changes in metabolic profiles caused by disruption of selected genes of secondary metabolism synthesis.

\section{Materials and methods}

\section{Chemicals}

Methanol (HPLC-grade) and $n$-hexane (p.a. grade) were purchased from Carl Roth GmbH \& Co. KG (Karlsruhe, Germany). Acetonitrile and methanol both LC-MS grade, were supplied by Th. Geyer GmbH \& Co. KG (Renningen, Germany), acetic acid (LC-MS grade) was purchased from Sigma-Aldrich GmbH (Steinheim, Germany).

\section{Fungal strains}

Ten fungal strains were used in this study, Botrytis cinerea B05.10 and three mutants derived from B05.10: B05.10pks6, B05.10bot2, B05.10pks6bot2 (Dalmais et al., 2011). Botrytis cinerea SAS405, Botrytis pseudocinerea VD110, Botrytis pseudocinerea VD184, Botrytis fabae 2240, Botrytis group Sa D08_H_8 and Botrytis group Sa G09_S33 (Leroch et al., 2013). The mutant strain B05.10pks6 does not produce botcinic acid, the mutant strain B05.10bot2 does not produce botrydial and the mutant strain B05.10pks6bot2 is neither botrydial nor botcinic acid producer 
(Dalmais et al., 2011).

\section{Culture conditions}

Twenty mL Gamborg's B5 medium (Gamborg et al., 1968) containing $10^{6}$ fungal spores of each strain was incubated in Petri dishes for 3 days in darkness at $24{ }^{\circ} \mathrm{C}$. Five Petri dishes for each treatment were prepared as replicates and all experiments were repeated for two times.

\section{Sample preparation}

The cultures were harvested and filtered. The supernatants were defatted with equal volumes of $n$-hexane for each sample. Then liquid extraction with an equal volume of ethyl acetate (EtOAc) was performed two times. The combined EtOAc extracts were dried using SpeedVac at $35{ }^{\circ} \mathrm{C}$ and resuspended in $2 \mathrm{~mL}$ of methanol/bi-distilled water (1:1). The solution was filtered through a $0.45-\mu \mathrm{m}$-pore-size filter (WICOM, Heppenheim, Germany). The solution was immediately subjected to HPLC-MS analysis or was stored at $-20{ }^{\circ} \mathrm{C}$. The mycelium was freeze dried and the dry weight was recorded.

\section{HPLC-MS based metabolic profiling}

For metabolite analysis, a HPLC-ESI-MS system (500-MS ion trap, Varian, Darmstadt, Germany) was used. The mobile phase of solvent A was $7 \mathrm{mM}$ acetic acid in $95 \%$ water $/ 5 \%$ acetonitrile, and solvent $\mathrm{B}$ was $7 \mathrm{mM}$ acetic acid in methanol. The binary gradient was started at $90 \%$ A for 5 min, continued for 25 min from $90 \%$ to $2 \%$ A, then kept for $8 \mathrm{~min}$ of $2 \%$ A for a washing step. The flow rate was $0.2 \mathrm{~mL} / \mathrm{min}$, $10 \mu \mathrm{L}$ of the sample was injected. This was followed by the re-equilibration steps using the starting conditions. The analytes were separated on a reversed-phase column (Polaris C18-Ether, 100 x 2 mm, $3 \mu \mathrm{m}$ particle size, Agilent, Darmstadt, Germany) at $40{ }^{\circ} \mathrm{C}$.

Both positive and negative ionization mode was used for non-targeted metabolite 
analysis (Ratzinger et al., 2009). The needle voltage was $-4,500 \mathrm{~V} /+5,000 \mathrm{~V}$, the shield voltage was $-600 \mathrm{~V} /+600 \mathrm{~V}$, the capillary voltage was $-/+40 \mathrm{~V}$. As drying gas nitrogen was used $(25 \mathrm{psi}, 172.5 \mathrm{kPa})$ at $250{ }^{\circ} \mathrm{C}$, and as nebulizing gas air was used (50 psi, $345 \mathrm{kPa}$ ). The ion trap was operated in full scan mode using the following parameters: mass range $m / z$ 100-1000, scan speed $5000 \mathrm{Da} / \mathrm{s}, 3$ scans averaged. Tandem mass spectrometry analysis was carried out by using turbo data dependent scanning (TurboDDS) with the same conditions.

\section{Data processing and statistical analysis}

For data acquisition, MS workstation/MS Data Review 6.9 (Varian) was used. Raw HPLC-MS data were processed with the Component Detection Algorithm (CODA, Windig et al., 1996) implemented in ACD/MS Manager v. 8.0 (Advanced Chemistry Development, Toronto, Canada). The CODA algorithm evaluates the quality of chromatographic peaks by calculating a mass quality index (MCQ) that reflects the similarity between the original mass chromatograms and their smoothed and mean-subtracted versions. Data processing by CODA included smoothing, baseline correction, and peak picking. Peak tables contained the monoisotopic mass (mass to charge ratio for $[\mathrm{M}-\mathrm{H}]^{-}$and $[\mathrm{M}+\mathrm{H}]^{+}$), retention time (Rt), peak area, and MCQ value for each signal that passed the MCQ thresholds of 0.8 and the S/N threshold of 10 . After peak alignment, signals occurring in controls (EtOAc extracts of un-inoculated medium) and signals detected in fewer than four of five replicates were discarded.

Multivariate analysis based on the LC-MS data was performed using PAST 3 for hierarchical cluster analysis (HCA). HCA was performed by calculation of Jaccard's similarity indices resulting in groups of samples show multivariate similarity in UPGMA clustering. The non-metric multidimensional scaling (MDS) was conducted using Primer 6 (Primer-E Ltd., Plymouth, UK). MDS was performed on basis of a Euclidean distance matrix. 


\section{Results and discussion}

\section{Comparison of metabolites produced by different Botrytis strains}

In order to evaluate the diversity of secondary metabolites produced by various Botrytis isolates which specialized to different host plants and identify the changes in metabolites that were caused by mutation of selected biosynthesis genes, the extractions of 3-day-old fungal cultures were analyzed for the production of secondary metabolites using mass spectrometry. Table 1 shows that the alignment of chromatograms without the use of $\mathrm{MS}^{2}$ and $\mathrm{MS}^{3}$, relying only on $\mathrm{m} / \mathrm{z}$ and retention time (RT). Mass signals originated from the solvent were discarded.

In total, 36 dominant metabolic signals were detected in positive mode and 18 metabolic signals in the negative mode. Among all the 50 metabolic signals, 27 metabolic signals were present in all the tested isolates. By the comparative analysis, four signals: $[\mathrm{M}+\mathrm{Na}]^{+} \mathrm{m} / z=333$ at $19.38 \mathrm{~min},[\mathrm{M}+\mathrm{Na}]^{+} \mathrm{m} / z=349$ at $21.55 \mathrm{~min}$, $[\mathrm{M}+\mathrm{Na}]^{+} \mathrm{m} / \mathrm{z}=277$ at $21.72 \mathrm{~min}$ and $[\mathrm{M}+\mathrm{Na}]^{+} \mathrm{m} / z=349$ at $24.66 \mathrm{~min}$ in the positive ionization mode, could be detected as corresponding ion in the negative mode yielding $[\mathrm{M}-\mathrm{H}]^{-} \mathrm{m} / z=309,[\mathrm{M}-\mathrm{H}]^{-} \mathrm{m} / z=325,[\mathrm{M}-\mathrm{H}]^{-} \mathrm{m} / z=253$ and $[\mathrm{M}-\mathrm{H}]^{-} \mathrm{m} / z=$ 325 , respectively. Two metabolite signals both have the $[\mathrm{M}+\mathrm{Na}]^{+} \mathrm{m} / \mathrm{z}=349$ and $[\mathrm{M}-\mathrm{H}]^{-} \mathrm{m} / \mathrm{z}=325$ while their retention time are different. They might represent as isomers. Many secondary metabolites present in low amounts were not included because they could not be identified due to the insufficient mass spectrum.

\section{Chemotaxonomy of Botrytis species}

The HPLC-MS profiles of all samples were transformed into a binary matrix showing the presence or absence of signals (Table 1). Similarities among aligned metabolic profiles for all cultures were investigated by MDS and HCA.

Similarities of the aligned metabolic profiles were explored by MDS (Figure 1). The Botrytis isolates were ordinated into three major groups. The two strains of B. pseudocinerea, VD110 and VD184, formed a separate group from B. cinerea 
B05.10 but not much from B. cinerea SAS405. Another distinct group comprised two B. cinerea BOT2 mutants, B05.10bot2 and B05.10pks6bot2. The third group contained B. cinerea wild-type B05.10 and its mutant B05.10pks6. The strawberry isolates $B$. group S D08_H_8 and B. group S G09_S33 showed similarities and were separated from the B. cinerea wild-type. The distance between SAS405 and B05.10, however, indicates that some variability has to be expected to exist between $B$. cinerea wild-type strains. Botrytis fabae, a distinctly different species, was clearly separated from all investigated $B$. cinerea wild type and mutant strains. As we can see from Figure 1, the metabolic signals which specifically produced by different groups of strains were labeled. A comparison of the metabolic profiles of B. pseudocinerea with other Botrytis strains showed that one metabolic signal with $\mathrm{m} / \mathrm{z} 338$ at $16.64 \mathrm{~min}$ in positive mode was specific for B. pseudocinerea. Furthermore, two signals with $\mathrm{m} / \mathrm{z}$ 189 and $\mathrm{m} / \mathrm{z} 233$ in negative ionization mode present only in B. fabae 2240 clearly separated B. fabae from other Botrytis species. The B. cinerea bot2 mutants group produced metabolic signals with $\mathrm{m} / \mathrm{z} 300.2,316.2$ and 332.5 in positive ionization mode which were the specific metabolites for this group. The metabolic signals with the $m / z, 966,217,487$ in positive mode and $\mathrm{m} / \mathrm{z}, 447$ in negative mode contribute to separate B. pseudocinerea and B. cinerea BOT2 mutants from the other Botrytis strains. The occurrence of the metabolite signals with $m / z 333$ in positive and $m / z 325$ and 371 in negative mode showed the similarity of $B$. group Sa with $B$. cinerea, while the $m / z 691$ and 325 which only detected in B. cinerea B05.10 and B05.10pks6 indicating the differences between $B$. cinerea and group Sa isolates.

By examining the HCA dendrogram (Figure 2), the Botrytis isolates were grouped into two major clusters (I and II). Each of these clusters was further divided into several subclusters. Cluster I comprised strains as B. cinerea B05.10 and B05.10pks6, Botrytis group Sa D08_H_8, Botrytis group Sa G09_S33 and B. fabae 2240. Cluster II contained strains as B. pseudocinerea isolates VD110 and VD184, B. cinerea mutant B05.10bot2, B05.10pks6bot2 and B. cinerea SAS405. In HCA dendrogram the subcluster of botrydial single mutant B05.10bot2 and the double mutant strain B05.10pks6bot2 group (bootstrap value, 99\%) and the subcluster of B. pseudocinerea 
isolates VD110 and VD184 (bootstrap value, 94\%) in cluster II was clustered away from the group of B. cinerea B05.10 and B05.10pks6 (bootstrap value, 92\%) in cluster I. The HCA result is in the agreement with the result of MDS analysis. Firstly, the result suggests that botrydial and its related metabolites play an important role in the secondary metabolite based classification, while on the other hand the inactivation of the BcPKS6 gene does not affect the metabolite production in the early stage. Secondly, the new genetic variant of B. cinerea, Botrytis group Sa which closely related to B. cinerea and B. fabae based on their sequence comparison (Leroch et al., 2013), shows a similar result in this study that Botrytis group Sa (D08_H_8 and G09_S33) were closely clustered with B. cinerea based on their metabolite profiles.

In summary, the metabolite profiles do not contradict the morphological character-based classification of the analyzed Botrytis strains and support further exploration of secondary metabolite profiles as useful classification tools.

Resemblance: D1 Euclidean distance

B. pseudocinerea VD184

2D Stress: 0.07

- B. pseudocinerea VD110

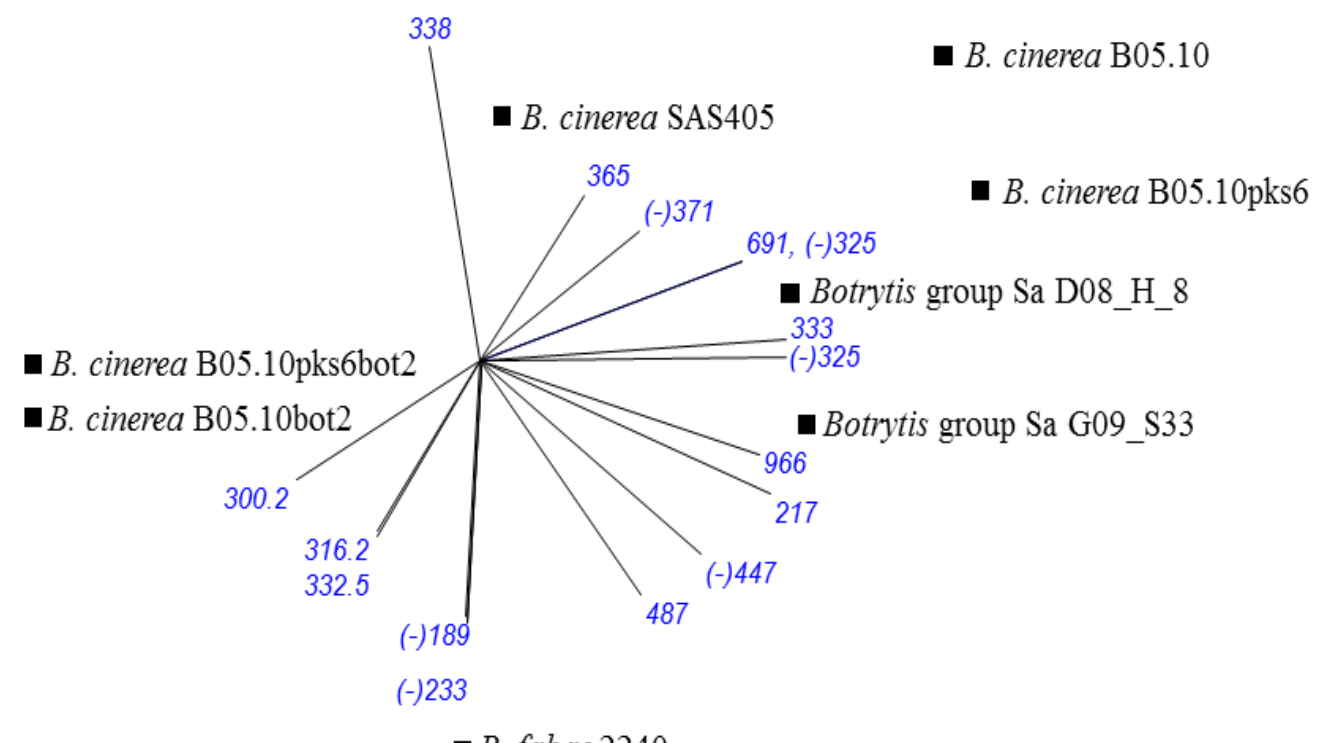

- B. fabae 2240

Figure 1. LC-MS peak-based non-metric multidimensional scaling (MDS) of Botrytis strain groups. Variable (peak signals) contributions that correlate with either of the MDS axes $(r>0.5)$ are indicated by lines. 


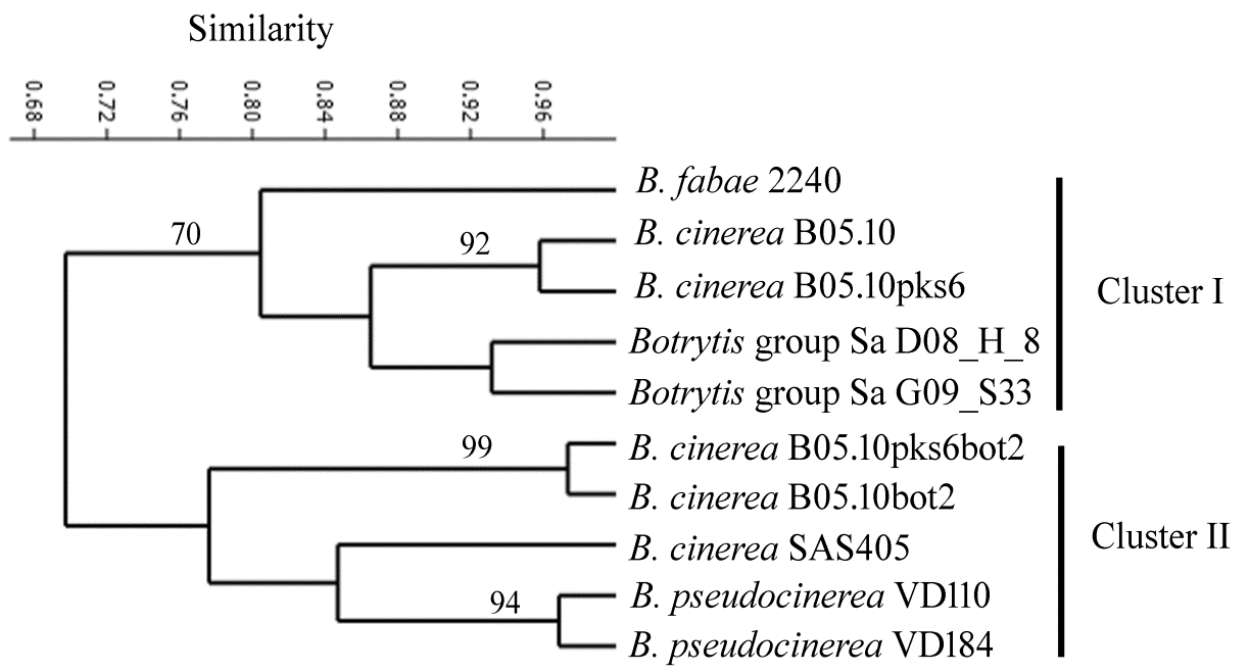

Figure 2. LC-MS peak-based hierarchical clustering analysis (HCA) of Botrytis strain groups (Jaccard's similarity coefficients). Bootstrap value $>70 \%$ for 1000 replicates are labeled.

\section{Comparison of metabolites produced by Botrytis cinerea B05.10 and its three} mutants

A comparison of the metabolic signals among B. cinerea B05.10 and its three mutants, B05.10pks6, B05.10bot2, and B05.10pks6bot2 revealed that B05.10 and B05.10pks6 have similar metabolic profiles, while BOT2 mutants display large metabolic differences.

As shown in Table 1, some of the metabolites produced by B. cinerea B05.10 and B05.10pks6 could not be found in BOT2 mutant B05.10bot2 and B05.10pks6bot2, such as metabolites with $\mathrm{m} / \mathrm{z}$ of $217,231,333,349,379,691,966$ in the positive mode and one signal with the $m / z$ of 371 in the negative mode. The BcBOT2 gene has been reported to be responsible for the first step of the botrydial (1) biosynthesis. Botrydial is a well-known and characteristic phytotoxin that is produced by $B$. cinerea. Inactivation of the BcBOT2 gene abolished production of botrydial and all related probotryane metabolites (Pinedo et al., 2008; Wang et al., 2009; Dalmais et al., 2011). Accordingly, we hypothesize that the metabolites which absent in both B05.10bot2 
and B05.10pks6bot2 strains but present in B05.10 and B05.10pks6 possibly represent botrydial and botrydial-related compounds.

The botrydial-related signals were further compared to published tandem mass spectrometry data. The metabolic signal of $\mathrm{m} / \mathrm{z} 333$ in positive mode at $19.38 \mathrm{~min}$ could also be ionized in the negative mode with $m / z=309[\mathrm{M}-\mathrm{H}]^{-}$. This suggested that the sodium adduct at $m / z=333[\mathrm{M}+\mathrm{Na}]^{+}$was formed in positive ion mode, the actual molecular weight of the compound is 310 . Its fragmentation product ions were signals at $m / z=273[\mathrm{M}+\mathrm{Na}-\mathrm{AcOH}]^{+}$in the positive mode and $m / z=249[\mathrm{M}-\mathrm{H}-\mathrm{AcOH}]^{-}$in the negative mode. According to the molecular weight and these additional fragmentation product ions, we assumed that this botrydial-related metabolites could be identified as botrydial (1), 8,9-epibotrydial (2), 1-epibotrydial (3) and 1,8,9-epibotrydial (4) all of which show signals of $m / z=310[\mathrm{M}]^{+}$in the mass spectrum and $m / z=250[\mathrm{M}-\mathrm{AcOH}]^{+}$in the tandem mass spectrum (Fehlhaber et al., 1974; Durán-Patrón et al., 1999).

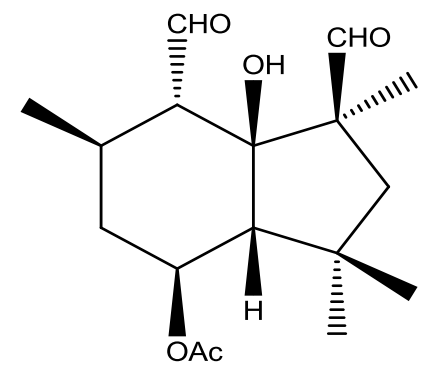

1

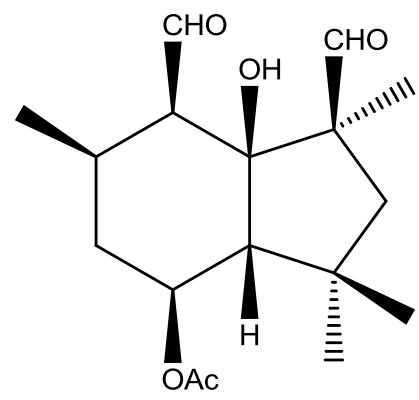

3

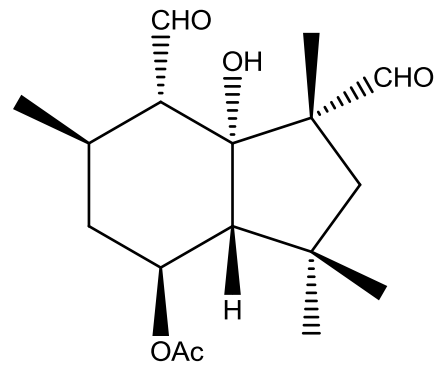

2

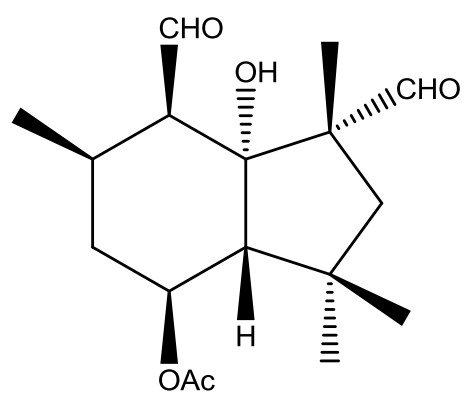

4 


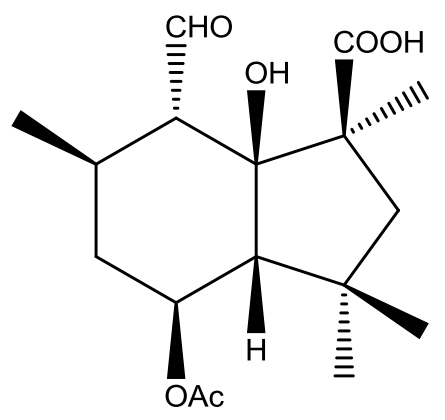

5

Two further botrydial-related signals which had the same $m / z=349[\mathrm{M}+\mathrm{Na}]^{+}$in the positive and $m / z=325[\mathrm{M}-\mathrm{H}]^{-}$in the negative mode at different retention times $(21.5$ and $24.6 \mathrm{~min}$ ) could be isomers. Both of their fragmentation product ions revealed signals at $m / z=265[\mathrm{M}-\mathrm{H}-\mathrm{AcOH}]^{-}$and $m / z=221\left[\mathrm{M}-\mathrm{H}-\mathrm{AcOH}-\mathrm{CO}_{2}\right]^{-}$in the negative ionization mode. Based on published spectra of botrydial derivatives with the molecular weight 326 and the identical additional fragmentation product ions, we proposed that one of the isomers could represent as botryaloic acid acetate (5) (Bradshaw and Hanson, 1980). Furthermore, the comparison with tandem mass spectrum in the negative ionization mode revealed that these isomers and another botrydial-related metabolite $(m / z=371)$ shared the same product ion at $m / z=221$, suggesting that these two metabolites might have a similar structure.

Conversely, B05.10bot2 and B05.10pks6bot2 showed four signals of $m / z$ 300.2, 316.2, 332 and 332.5 that were absent in B05.10 and B05.10pks6. The gene knockout may have affected these metabolic pathways. Pinedo et al. (2008) reported that the overproduction of the botcinic acid derivatives occurs in the B05.10bot2 mutants but to a lesser extent in the wild-type strain. We assume that these four metabolites might be the botcinic acid related metabolites or some novel natural products caused by the gene mutation. To confirm this further studies are required.

The B. cinerea mutants B05.10pks6 and B05.10pks6bot2 have been demonstrated that did not produce botcinic acid and its derivatives due to the inactivation of the BcPKS6 gene as suggested by previous studies (Dalmais et al., 2011). Therefore, the metabolites which were produced by the wild-type strain B05.10 but not in the 
BcPKS6 mutants B05.10pks6 and B05.10pks6bot2 can be assigned tentatively as botcinic acid or some of its derivatives. Unfortunately, the comparison of the metabolite data of $\mathrm{B} 05.10$ and its mutant B05.10pks6 provided no further hints for structure assignment.

For this investigation, the fungal growth was restricted to 3 days after spore germination because in later growth stages the cultures differentiated by producing an aerial mycelium. Three days after germination the mycelia covered the bottom of the Petri dishes with a homogeneous layer. Consequently, we decided to limit the incubation to three days. The comparison of the $B$. cinerea wild-type strain with its three mutants revealed that the inactivation of BcBOT2 gene successfully affected the production of the metabolites while BcPKS6 gene inactivation did not. This indicates that the incubation condition we used in this work may not been suitable for monitoring the biosynthesis of botcinic acid and its derivatives.

\section{Comparison of Botrytis cinerea biomass}

The fungal biomass of the tested Botrytis strains are shown in Figure 3. In order to check the effects of botrydial and botcinic acid on the growth of B. cinerea, we compared the biomass of the wild-type strain B05.10 to its mutant strains (B05.10pks6, B05.10pks6bot2 and B05.10bot2). As shown in Figure 3, the single PKS6 mutant strain grew quicker than the wild-type strain B05.10 and the other mutant strains. The B05.10pks6bot2 and B05.10bot2 showed slightly slower growth rate than the wild-type strain B05.10. In previous work, the growth rate of wild-type strain B05.10 and its single botcinic acid mutants (B05.10pks6 and B05.10pks9), the single botrydial mutant (B05.10bot2) and double mutants B05.10pks6bot2 have been observed, which revealed a slower growth rate of the double mutants than the wild-type and single mutants. The author of this study suggested that botrydial and botcinic acid might play an unexpected redundant role in saprophytic growth (Dalmais et al., 2011). Surprisingly, the fungal growth rate of the botrydial mutants B05.10pks6bot2 and B05.10bot2 in this study were also slower than the wild-type 
strain B05.10, which supports the previous findings that the phytotoxin botrydial might cause effect in saprophytic growth. The similar metabolite profiles of B05.10 and B05.10pks6 let expect similar growth rates of these two strains, while the mutant strain B05.10pks6 grew quicker than B05.10. We assumed that B05.10pks6 needs more energy for biosynthesis of some metabolites which are in the mycelium but not visible in the fungal culture.

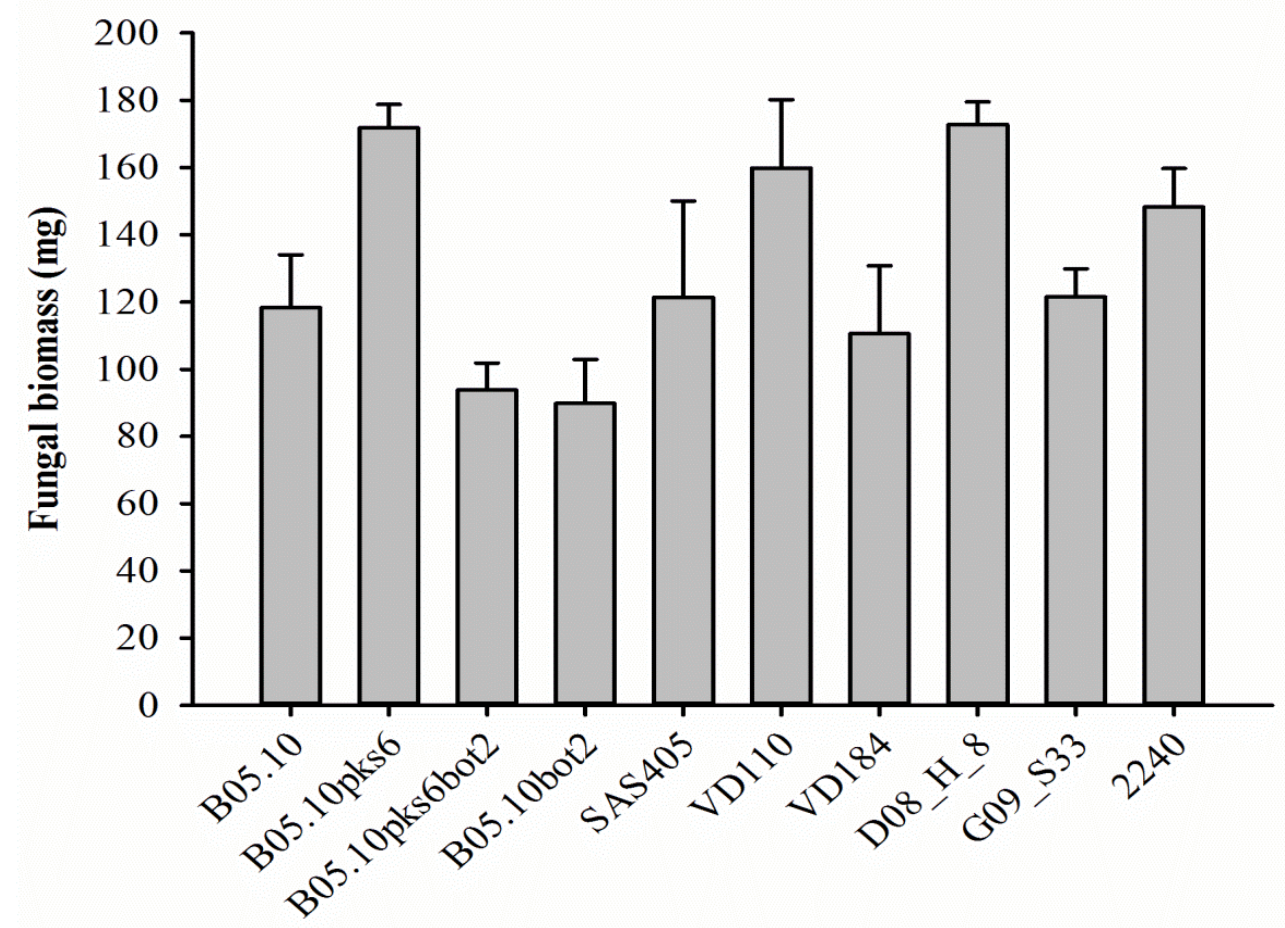

Figure 3. Fungal biomass of Botrytis strains after 3 days incubated in Gamborg's B5 medium. B05.10: Botrytis cinerea wild-type; B05.10pks6: Botrytis cinerea mutant do not produce botcinic acid; B05.10bot2: Botrytis cinerea mutant do not produce botrydial; B05.10pks6bot2: Botrytis cinerea mutant do not produce botrydial and botcinic acid; SAS405: Botrytis cinerea SAS405; VD110: Botrytis pseudocinerea VD110; VD184: Botrytis pseudocinerea VD184; D08_H_8: Botrytis group Sa D08_H_8; G09_S33: Botrytis group Sa G09_S33; 2240: Botrytis fabae 2240.

In conclusion, non-targeted and targeted metabolic profiling has been applied successfully in characterizing metabolite patterns of plants and other organisms. Predominately, HPLC or GC coupled with MS, UV or NMR were used (Baniasadi et 
al., 2013, Denkert et al., 2006; Honoré et al., 2013). Here we presented results that illustrate the applicability of the methodology for metabolic characterization of fungal strains, specifically for Botrytis species. The obtained metabolite patterns using LC-MS metabolic profiling technique strongly supported the classification of tested Botrytis which was proposed on basis of their morphological characteristics. For the new clade, Botrytis group Sa, metabolic profiling suggests close relationship with B. cinerea. In addition, the comparison of $B$. cinerea and its mutants allowed some tentative identification of the detected metabolites. More structural information, however, warrants further studies. This study provide further support for applying LC-MS-based metabolic profiling for the chemotaxonomic characterization of fungal strains (Kang et al., 2011; Kim et al., 2012; Lee et al., 2013; Park et al., 2013). Methodically, this work provides some evidence in support of turbo data dependent scanning (TurboDDS) as metabolic profiling evaluation tools that automatically performed $\mathrm{MS}^{\mathrm{n}}$ scans on peaks as they elute from the column. The use of TurboDDS could be a useful way to confirm the structure of the known or unknown compounds in the metabolomics approaches, and the MSMS data provided more reliable information for metabolic analysis. Our study, however, also points to the fact that the comparison among fungal strains should cover all growth phases and physiological stages. In our further work the optimization of the incubation conditions will be included and the work towards the identification of the species-specific metabolites is under way. 
Table 1. Secondary metabolite signals of 10 Botrytis strains in 3-day-old culture detected by HPLC-MS ${ }^{1}$.

\begin{tabular}{|c|c|c|c|c|c|c|c|c|c|c|c|c|}
\hline \multirow{2}{*}{$\begin{array}{l}\text { Retention } \\
\text { time } \\
(\mathrm{min})\end{array}$} & \multirow{2}{*}{$\begin{array}{c}m / z \\
(+)\end{array}$} & \multirow{2}{*}{$\begin{array}{c}m / z \\
(-)\end{array}$} & \multicolumn{2}{|c|}{$\begin{array}{c}\text { Botrytis } \\
\text { pseudocinerea }\end{array}$} & \multicolumn{5}{|c|}{ Botrytis cinerea } & \multicolumn{2}{|c|}{ Botrytis group Sa } & \multirow{2}{*}{$\begin{array}{c}\begin{array}{c}\text { Botrytis } \\
\text { fabae }\end{array} \\
2240\end{array}$} \\
\hline & & & VD110 & VD184 & B05.10 & $\begin{array}{c}\text { B05.10 } \\
\text { pks6 }\end{array}$ & $\begin{array}{c}\text { B05.10 } \\
\text { pks6bot2 }\end{array}$ & $\begin{array}{c}\text { B05.10 } \\
\text { bot } 2\end{array}$ & SAS405 & D08_H_8 & G09_S33 & \\
\hline 1.87 & 243 & & $\mathrm{X}^{\mathrm{a}}$ & $\mathrm{X}$ & $X$ & $X$ & $X$ & $X$ & $\mathrm{X}$ & $X$ & $X$ & $X$ \\
\hline 2 & 200 & & $\mathrm{X}$ & $X$ & $X$ & $X$ & $X$ & $X$ & $X$ & $X$ & $X$ & $\mathrm{X}$ \\
\hline 2.11 & 290 & & $X$ & $X$ & $X$ & $X$ & $X$ & $X$ & $X$ & $X$ & $X$ & $X$ \\
\hline 2.12 & & 181 & $X$ & $X$ & $X$ & $X$ & $X$ & $X$ & $X$ & $X$ & $X$ & $X$ \\
\hline 2.13 & 316 & & $X$ & $X$ & $X$ & $X$ & $X$ & $X$ & $X$ & $X$ & $X$ & $X$ \\
\hline 2.99 & 272 & & $X$ & $X$ & $X$ & $X$ & $X$ & $X$ & $X$ & $X$ & $X$ & $X$ \\
\hline 3.45 & 275 & & & & & $\mathrm{X}$ & & & & & & \\
\hline 3.6 & 330 & & $X$ & $X$ & $X$ & $X$ & $X$ & $X$ & $X$ & $X$ & $X$ & $X$ \\
\hline 3.71 & 300.2 & & $X$ & $X$ & $X$ & $\mathrm{X}$ & $X$ & $\mathrm{X}$ & $\mathrm{X}$ & $X$ & $\mathrm{X}$ & $\mathrm{X}$ \\
\hline 3.71 & & 117 & $X$ & $X$ & $X$ & $X$ & $X$ & $X$ & $X$ & $X$ & $X$ & $X$ \\
\hline 8.58 & & 193.5 & $X$ & $X$ & $X$ & $X$ & $X$ & $X$ & $X$ & $X$ & $X$ & $X$ \\
\hline 9.4 & 295 & & $X$ & $X$ & $X$ & $X$ & $X$ & $X$ & $X$ & $X$ & $X$ & $X$ \\
\hline 10.51 & & 186 & $X$ & $X$ & $X$ & $X$ & $\mathrm{X}$ & $\mathrm{X}$ & $\mathrm{X}$ & $\mathrm{X}$ & $X$ & $\mathrm{X}$ \\
\hline 11.52 & 713 & & $X$ & $X$ & $X$ & $X$ & $X$ & $X$ & $X$ & $X$ & $X$ & $X$ \\
\hline 11.65 & 357 & & $X$ & $X$ & $X$ & $X$ & $X$ & $X$ & $X$ & $X$ & $X$ & $X$ \\
\hline 12.57 & 333 & & $X$ & $X$ & $X$ & $X$ & $X$ & $X$ & $X$ & $X$ & $X$ & $X$ \\
\hline 13.85 & 318 & & $X$ & $X$ & $X$ & $X$ & $X$ & $X$ & $X$ & $X$ & $X$ & $X$ \\
\hline 14.05 & 321 & 297 & $X$ & $X$ & $X$ & $X$ & $X$ & $X$ & $X$ & $X$ & $X$ & $X$ \\
\hline 14.2 & & 356 & $X$ & $X$ & $X$ & $X$ & $X$ & $X$ & $X$ & $X$ & $X$ & $X$ \\
\hline
\end{tabular}


Table 1. continued

\begin{tabular}{|c|c|c|c|c|c|c|c|c|c|c|c|c|}
\hline \multirow{2}{*}{$\begin{array}{l}\text { Retention } \\
\text { time } \\
(\text { min })\end{array}$} & \multirow{2}{*}{$\begin{array}{l}m / z \\
(+)\end{array}$} & \multirow{2}{*}{$\begin{array}{c}m / z \\
(-)\end{array}$} & \multicolumn{2}{|c|}{$\begin{array}{c}\text { Botrytis } \\
\text { pseudocinerea }\end{array}$} & \multicolumn{5}{|c|}{ Botrytis cinerea } & \multicolumn{2}{|c|}{ Botrytis group Sa } & \multirow{2}{*}{$\begin{array}{c}\begin{array}{c}\text { Botrytis } \\
\text { fabae }\end{array} \\
2240\end{array}$} \\
\hline & & & VD110 & VD184 & B05.10 & $\begin{array}{c}\text { B05.10 } \\
\text { pks6 }\end{array}$ & $\begin{array}{c}\text { B05.10 } \\
\text { pks6bot2 }\end{array}$ & $\begin{array}{c}\text { B05.10 } \\
\text { bot2 }\end{array}$ & SAS405 & D08_H_8 & G09_S33 & \\
\hline 15.23 & 332.5 & & & & & & $\mathrm{X}$ & $\mathrm{X}$ & & & $X$ & \\
\hline 15.52 & & 189 & & & & & & & & & & $X$ \\
\hline 15.72 & 332 & & & & & & $X$ & $X$ & & & & \\
\hline 15.8 & 217 & & & & $\mathrm{X}$ & & & & & & & \\
\hline 16.5 & 316.2 & & & & & & $\mathrm{X}$ & $\mathrm{X}$ & & & $\mathrm{X}$ & \\
\hline 16.64 & 338 & & $X$ & $X$ & & & & & $\mathrm{X}$ & & & \\
\hline 19.38 & 333 & 309 & & & $X$ & $X$ & & & & $X$ & $X$ & \\
\hline 20.69 & 365 & & $X$ & $X$ & $\mathrm{X}$ & $\mathrm{X}$ & $X$ & & & $\mathrm{X}$ & $\mathrm{X}$ & \\
\hline 21.21 & 691 & & & & $\mathrm{X}$ & $\mathrm{X}$ & & & & & & \\
\hline 21.29 & 231 & & & & $\mathrm{X}$ & $\mathrm{X}$ & & & & & & \\
\hline 21.54 & 349 & 325 & & & $\mathrm{X}$ & $\mathrm{X}$ & & & $\mathrm{X}$ & & & \\
\hline 21.72 & 277 & 253 & $X$ & $X$ & $\mathrm{X}$ & $\mathrm{X}$ & $X$ & $X$ & $\mathrm{X}$ & $X$ & $\mathrm{X}$ & $X$ \\
\hline 21.87 & & 335 & $\mathrm{X}$ & $\mathrm{X}$ & $\mathrm{X}$ & $X$ & $\mathrm{X}$ & $\mathrm{X}$ & $\mathrm{X}$ & $\mathrm{X}$ & $X$ & $\mathrm{X}$ \\
\hline 22.02 & & 209 & $\mathrm{X}$ & $\mathrm{X}$ & $\mathrm{X}$ & $\mathrm{X}$ & $\mathrm{X}$ & $\mathrm{X}$ & $\mathrm{X}$ & $X$ & $X$ & $X$ \\
\hline 22.77 & 487 & & & & $X$ & $X$ & $X$ & $X$ & $X$ & $X$ & $X$ & $X$ \\
\hline 22.92 & & 447 & & & $X$ & $X$ & $X$ & $X$ & & $X$ & $X$ & $X$ \\
\hline 23.4 & 379 & & & & $X$ & $X$ & & & & $X$ & $X$ & $X$ \\
\hline 24.37 & & 489 & & & $X$ & $X$ & $X$ & $X$ & $X$ & $X$ & $X$ & $X$ \\
\hline 24.56 & 217 & & & & $X$ & $X$ & & & & $X$ & $X$ & $X$ \\
\hline
\end{tabular}


Table 1. continued

\begin{tabular}{|c|c|c|c|c|c|c|c|c|c|c|c|c|}
\hline \multirow{2}{*}{$\begin{array}{l}\text { Retention } \\
\text { time } \\
(\mathrm{min})\end{array}$} & \multirow{2}{*}{$\begin{array}{l}m / z \\
(+)\end{array}$} & \multirow{2}{*}{$\begin{array}{c}m / z \\
(-)\end{array}$} & \multicolumn{2}{|c|}{$\begin{array}{c}\text { Botrytis } \\
\text { pseudocinerea }\end{array}$} & \multicolumn{5}{|c|}{ Botrytis cinerea } & \multicolumn{2}{|c|}{ Botrytis group Sa } & \multirow{2}{*}{$\begin{array}{r}\begin{array}{r}\text { Botryti } \\
\text { fabae }\end{array} \\
2240\end{array}$} \\
\hline & & & VD110 & VD184 & B05.10 & $\begin{array}{c}\text { B05.10 } \\
\text { pks6 }\end{array}$ & $\begin{array}{c}\text { B05.10 } \\
\text { pks6bot2 }\end{array}$ & $\begin{array}{c}\text { B05.10 } \\
\text { bot2 }\end{array}$ & SAS405 & D08_H_8 & G09_S33 & \\
\hline 25.63 & & 371 & & & $X$ & $\mathrm{X}$ & & & & $X$ & $X$ & \\
\hline 26 & 335 & & $X$ & X & $X$ & $X$ & $X$ & $X$ & $X$ & $X$ & $X$ & $X$ \\
\hline 26.8 & 318 & & $\mathrm{X}$ & $\mathrm{X}$ & $\mathrm{X}$ & $\mathrm{X}$ & $X$ & $\mathrm{X}$ & $X$ & $\mathrm{X}$ & $\mathrm{X}$ & $\mathrm{X}$ \\
\hline 27.19 & 318 & & $X$ & X & $X$ & $X$ & $X$ & $X$ & $X$ & $X$ & $X$ & $X$ \\
\hline 28.37 & & 205 & & $\mathrm{X}$ & $\mathrm{X}$ & $\mathrm{X}$ & $X$ & $\mathrm{X}$ & $X$ & $\mathrm{X}$ & & $X$ \\
\hline 29.26 & 816 & & $X$ & $X$ & $X$ & X & $X$ & $X$ & $X$ & $X$ & $X$ & $X$ \\
\hline 30.99 & 540 & & $X$ & $X$ & $X$ & $\mathrm{X}$ & $X$ & $\mathrm{X}$ & $X$ & $X$ & $X$ & $X$ \\
\hline 31.66 & 282 & & $X$ & $X$ & $X$ & $X$ & $X$ & $X$ & $X$ & $X$ & $X$ & $X$ \\
\hline 32.59 & 966 & & & & $X$ & $X$ & & & $X$ & $X$ & $X$ & $X$ \\
\hline
\end{tabular}

${ }^{\mathrm{T}}$ Criteria for analysis: minimum intensity for negative ionization 1,000 cpm, for positive ionization 5,000 cpm; MCQ 0.8; relative standard deviation among replicates $<1$.

${ }^{\mathrm{a}} \mathrm{X}$ : detected peak signal 


\section{References}

Baniasadi, H., Gowda, G.A.N., Gu, H., Zeng, A., Zhuang, S., Skill, N., Maluccio, M., Raftery, D., 2013. Targeted metabolic profiling of hepatocellular carcinoma and hepatitis C using LC-MS/MS. Electrophoresis 34, 2910-2917.

Bradshaw, A.P.W., Hanson, J.R., 1980. Three new sesquiterpenoid metabolites of Botrytis cinerea. J. Chem. Soc. [Perkin 1] 741-743.

Cimmino, A., Villegas-Fernández, A.M., Andolfi, A., Melck, D., Rubiales, D., Evidente, A., 2011. Botrytone, a new naphthalenone pentaketide produced by Botrytis fabae, the causal agent of chocolate spot disease on Vicia faba. J. Agric. Food Chem. 59, 9201-9206.

Dalmais, B., Schumacher, J., Moraga, J., Le Pecheur, P., Tudzynski, B., Collado, I.G., Viaud, M., 2011. The Botrytis cinerea phytotoxin botcinic acid requires two polyketide synthases for production and has a redundant role in virulence with botrydial. Mol. Plant Pathol. 12, 564-579.

Dean, R., Van Kan, J.A.L., Pretorius, Z.A., Hammond-kosack, K.E., Di Pietro, A., Spanu, P.D., Rudd, J.J., Dickman, M., Kahmann, R., Ellis, J., Foster, G.D., 2012. The top 10 fungal pathogens in molecular plant pathology. Mol. Plant Pathol. 13, 414-430.

Debieu, D., Bach, J., Montesinos, E., Fillinger, S., Leroux, P., 2013. Role of sterol 3-ketoreductase sensitivity in susceptibility to the fungicide fenhexamid in Botrytis cinerea and other phytopathogenic fungi. Pest Manag. Sci. 69, 642651.

Denkert, C., Budczies, J., Kind, T., Weichert, W., Tablack, P., Sehouli, J., Niesporek, S., Könsgen, D., Dietel, M., Fiehn, O., 2006. Mass spectrometry-based metabolic profiling reveals different metabolite patterns in invasive ovarian carcinomas and ovarian borderline tumors. Cancer Res. 66, 10795-10804.

Durán-Patrón, R., Hernández-Galán, R., Rebordinos, L.G., Cantoral, J.M., Collado, I.G., 1999. Structure-activity relationships of new phytotoxic metabolites with 
the botryane skeleton from Botrytis cinerea. Tetrahedron 55, 2389-2400.

Fehlhaber, H.W., Geipel, R., Mercker, H.J., Tschesche, R., Welmar, K., Schönbeck, F., 1974. Botrydial, ein sesquiterpen-antibiotikum aus der Nährlösung des Pilzes Botrytis cinerea. Chem. Ber. 107, 1720-1730.

Fischedick, J.T., Hazekamp, A., Erkelens, T., Choi, Y.H., Verpoorte, R., 2010. Metabolic fingerprinting of Cannabis sativa L., cannabinoids and terpenoids for chemotaxonomic and drug standardization purposes. Phytochemistry 71, 2058-2073.

Gamborg, O.L., Miller, R.A., Ojima, O., 1968. Nutrient requirements of suspension cultures of soybean root cell. Exp. Cell Res. 50, 151-158.

Hanhineva, K., Kokko, H., Siljanen, H., Rogachev, I., Aharoni, A., Kärenlampi, S.O., 2009. Stilbene synthase gene transfer caused alterations in the phenylpropanoid metabolism of transgenic strawberry (Fragaria $\times$ ananassa). J. Exp. Bot. 60, 2093-2106.

Harrison, J.G., 1983. Distinguishing between lesions caused by Botrytis fabae and B. cinerea on field bean leaves. Trans. Br. Mycol. Soc. 81, 663-664.

Heinrich, J., Valentova, K., Vacek, J., Palikova, I., Zatloukalova, M., Kosina, P., Ulrichova, J., Vrbkova, J., Simanek, V., 2013. Metabolic profiling of phenolic acids and oxidative stress markers after consumption of Lonicera caerulea $\mathrm{L}$. fruit. J. Agric. Food Chem. 61, 4526-4532.

Honoré, A., Thorsen, M., Skov, T., 2013. Liquid chromatography-mass spectrometry for metabolic footprinting of co-cultures of lactic and propionic acid bacteria. Anal. Bioanal. Chem. 1-20.

Jacyno, J.M., Harwood, J.S., Cutler, H.G., Dulik, D.M., 1994. Structure and solution-state conformation of botcinolide, a new biologically active metabolite from the fungus Botrytis cinerea. Int. J. Rapid Publ. Crit. 50, $11585-11592$.

Kang, D., Kim, J., Choi, J.N., Liu, K.H., Lee, C.H., 2011. Chemotaxonomy of Trichoderma spp. using mass spectrometry-based metabolite profiling. J. 
Microbiol. Biotechnol. 21, 5-13.

Kim, J., Choi, J. N., Kim, P., Sok, D. E., Nam, S. W., and Lee, C. H., 2009. LC-MS/MS profiling-based secondary metabolite screening of Myxococcus xanthus. J. Microbiol. Biotechnol. 19, 51- 54.

Lee, M.Y., Park, H.M., Son, G.H., Lee, C.H., 2013. Liquid chromatography-mass spectrometry-based chemotaxonomic classification of Aspergillus spp. and evaluation of the biological activity of its unique metabolite, neosartorin. J. Microbiol. Biotechnol. 23(7), 932 - 941.

Leroch, M., Plesken, C., Weber, R.W.S., Kauff, F., Scalliet, G., Hahn, M., 2013. Gray mold populations in German strawberry fields are resistant to multiple fungicides and dominated by a novel clade closely related to Botrytis cinerea. Appl. Environ. Microbiol. 79, 159-167.

Park, S.Y., Lim, S.H., Ha, S.H., Yeo, Y., Park, W.T., Kwon, D.Y., Park, S.U., Kim, J.K., 2013. Metabolite profiling approach reveals the interface of primary and secondary metabolism in colored cauliflowers (Brassica oleracea L. ssp. botrytis). J. Agric. Food Chem. 61, 6999-7007.

Pinedo, C., Wang, C.M., Pradier, J.M., Dalmais, B., Choquer, M., Le Pêcheur, P., Morgant, G., Collado, I.G., Cane, D.E., Viaud, M., 2008. Sesquiterpene synthase from the botrydial biosynthetic gene cluster of the phytopathogen Botrytis cinerea. ACS Chem. Biol. 3, 791-801.

Ratzinger, A., Riediger, N., von Tiedemann, A., Karlovsky, P., 2009. Salicylic acid and salicylic acid glucoside in xylem sap of Brassica napus infected with Verticillium longisporum. J. Plant Research 122, 571-579.

Torres, A.M., Román, B., Avila, C.M., Satovic, Z., Rubiales, D., Sillero, J.C., Cubero, J.I., Moreno, M.T., 2006. Faba bean breeding for resistance against biotic stresses: towards application of marker technology. Euphytica 147, 67-80.

Walker, A.S., Gautier, A., Confais, J., Martinho, D., Viaud, M., Le Pêcheur, P., Dupont, J., Fournier, E., 2011. Botrytis pseudocinerea, a new cryptic species causing gray mold in French vineyards in sympatry with Botrytis cinerea. 
Phytopathology 101, 1433-1445.

Wang, C.M., Hopson, R., Lin, X., Cane, D.E., 2009. Biosynthesis of the sesquiterpene botrydial in Botrytis cinerea. Mechanism and stereochemistry of the enzymatic formation of presilphiperfolan-8 $\beta$-ol. J. Am. Chem. Soc. 131, 8360-8361.

Washington, W.S., 1993. Recent advances in Botrytis research. Australas. Plant Pathol. $22,156-157$.

Welmar, K., Tschesche, R., Breitmaier, E., 1979. Botrylacton, ein neuer Wirkstoff aus der Nährlösung des Pilzes Botrytis cinerea, 2. Chem. Ber. 112, 3598-3602.

Windig, W., Phalp, J.M., Payne, A.W., 1996. Noise and background reduction method for component detection in liquid chromatography-mass spectrometry. Anal. Chem. 68, 3602-3606. 


\section{Chapter 5: General discussion}

The overall aim of this work was to study secondary metabolites in fungal biotic interactions. The first objective was to investigate the production of secondary metabolites in the fungus-fungus interaction. For this purpose, we co-cultivated Fusarium verticillioides and Gliocladium roseum for the investigation of secondary metabolites involving mycotoxins. The second objective was to explore secondary metabolites produced by various isolates of Botrytis that specialized to different host plants, and to highlight the changes in metabolic profiles caused by disruption of selected genes of secondary metabolism synthesis.

\section{Secondary metabolites in fungal interaction}

Recent research on fungal biotic interactions indicate that co-culturing fungi with other microbes could produce numbers of novel metabolites (Cueto et al., 2001; Scherlach and Hertweck, 2009; Bertrand et al., 2013), change the yields of known secondary metabolites (Oh et al., 2007) and could also generate the analogues of known metabolites by combination pathways (Degenkolb et al., 2002). While up to now, most of the research reports on fungal interactions were mainly focused on the discovery of novel metabolites which show potentially useful properties (Cueto et al., 2001; Zuck et al., 2011; Li et al., 2011; Bertrand et al., 2013). Only few works systematically studied the origin and the biological role of the production of fungal metabolites in the biotic interactions (Pettit, 2009; Schroeckh et al., 2009).

In this study, the production of secondary metabolites and the fungal growth affected by the co-cultivation of phytopathogen $F$. verticillioides and mycoparasite G. roseum were investigated systematically for the first time. Metabolic profiling generated by HPLC-MS in this study revealed a dramatic and unprecedented increase in the diversity of secreted metabolites in dual fungal cultures compared with the single cultures. Some of them are likely to have originated from new structures which have not been isolated yet from these organisms. The fungal growth was monitored by using real-time PCR technique which is an effective tool to detect variation between fungal single and co-cultures. Previous reports about the co-culture of 
microorganisms showed that the silent gene clusters for expressing bioactive secondary metabolites in the competitive environmental conditions could be activated (Pettit, 2009; Schroeckh et al., 2009). Hence, we assume that the changes of the secondary metabolites production in our co-cultures could be a response to their biotic challenge. Under the competitive conditions, the fungal strains possibly synthesize a large set of metabolites with different modes of action to increase the chance that some of these metabolites will suppress the competitor.

Two new metabolites 2, 3 and one new natural product 1, together with a known fusaric acid derivative 4 were first isolated from the dual cultures of $F$. verticillioides and G. roseum. Our results confirmed that the co-cultivation of two or more microbes is an efficient method for the discovery of new structures (Bertrand et al., 2013; Cueto et al., 2001; Park et al., 2009). The production of these metabolites in the fungal dual cultures lead the suspection of the reason for synthesize pathway. Surprisingly, we found that compound $\mathbf{2}$ and $\mathbf{3}$ are the biotransformation products of fusaric acid by G. roseum under our growth condition, while the origination of compound $\mathbf{1}$ and compound $\mathbf{4}$ are still not really clear. The antifungal activity assay showed that the compounds 1-3, especially compound $\mathbf{2}$ are less toxic than fusaric acid towards Botrytis cinerea and Aspergillus niger. The significantly reduced antifungal activity of compound 2 when compared with fusaric acid in this study supported the previous indication that the carboxylic acid in fusaric acid appeared to be necessary for the toxic activity (Crutcher et al., 2014). Detoxification of fusaric acid by using different microorganisms have been carried out previously (Utsumi et al., 1988; Fakhouri et al., 2003). Crutcher et al. (2014) recently reported that the fusaric acid converted to a less phytotoxic product fusarinol by Aspergillus tubingensis. On the other hand the fungal isolate $G$. roseum had been proved to possess the ability to transform nigranoic acid into three new products and make hydroxylation of sesquiterpene into several derivatives (Dong et al., 2007; García-Granados et al., 2001 and 2002). Here we report for the first time that $G$. roseum was able to detoxify fusaric acid into two new fusaric acid derivatives $\mathbf{2}$ and $\mathbf{3}$. Although fusaric acid could be converted to fusaric acid derivatives by $G$. roseum, the production of fusaric acid in the co-cultures was not significantly affected by $G$. roseum. We assume that during the time when $G$. roseum detoxified fusaric acid, the fungus $F$. verticillioides kept producing fusaric acid simultaneously in the interaction cultures for surviving in this competitive environment. Accordingly, the four compounds are likely to originate from the fusaric 
acid-type precursors during the interaction. We emphasize that fusaric acid might play an important role in this competitive interaction.

Furthermore, $F$. verticillioides is well known to produce biologically active secondary metabolites, including the mycotoxin fumonisins (Brown et al., 2012). The effect of co-incubate $F$. verticillioides with Aspergillus spp. on fumonisins production was studied by Velluti et al. and Marin et al., although fumonisin concentrations were not normalized by fungal biomass (Velluti et al., 2000; Marín et al., 2001). As a whole, the fumonisin levels in mixed cultures were depended on the condition of the cultures, which may be stimulated or suppressed in the co-cultures. Rodriguez Estrada et al. (2011) observed a slight suppression of fumonisin levels normalized by biomass in interactions between $F$. verticillioides and $U$. maydis. They assumed that fumonisins may have limited the growth of $U$. maydis in dual cultures with $F$. verticillioides. In our study, the amount of fumonisin B1, B2 and B3 produced by F. verticillioides were reduced in $F$. verticillioides and $G$. roseum dual cultures compared to $F$. verticillioides single culture. The results of FB1 biotransformation by $G$. roseum suggest that the degradation or biotransformation was not the reason for the reduction of fumonisin B1 in the dual culture. A likely reason of the reduced fumonisin B production in dual cultures would be the inhibition of the growth of $F$. verticillioides by the mycoparasitic fungus $G$. roseum.

Besides the investigation on secondary metabolites, we monitored the changes of the fungal growth during the interaction of $F$. verticillioides with G. roseum. Interestingly, we found that the biomass of $F$. verticillioides declined in dual cultures with $G$. roseum, suggesting that the mycelium of $F$. verticillioides was destroyed by mycoparasitism. Slow replication and a reduced metabolic rate may have induced an "antibiotic-tolerant" state, as recently shown for Mycobacterium tuberculosis (Baek et al. 2011). We speculate that in the interaction cultures, G. roseum activated its internal emergency response mechanisms which were enabled it to cope with interference competition metabolites produced by $F$. verticillioides.

Efforts to obtain antagonistic microorganisms effectively against plant pathogens have been underway in recent years. The mechanism of microbial derived control agent has been characterized with four modes by which a bioagent can effectively act against pathogen: (1) a source of toxic antibiotic substances, (2) a competitor for the survival food, (3) a parasitic attack against pathogen, or (4) an induced indirect toxic effect by the release of volatile compounds. Therefore, firstly our results indicated that the 
application of $G$. roseum as a biological control agent against $F$. verticillioides may reduce the fumonisins levels in the commodity. Secondly, the toxin fusaric acid was detoxified by bioagent strain $G$. roseum through its owner metabolic system. As such, $G$. roseum could be considered as a biocontrol agent against $F$. verticillioides under the laboratory conditions that provides a promising approach for the further research.

\section{Metabolic profiling approaches in fungal secondary metabolites}

HPLC-MS based metabolic profiling method was used in this thesis to reveal a dramatic and unprecedented increase in the diversity of secreted metabolites in dual fungal cultures, as well as to explore the variation in secondary metabolites produced by different Botrytis isolates and the changes in metabolic profiles caused by disruption of selected genes of secondary metabolism synthesis.

During last decades, analytical chemistry developed fast together with the development of analytical instruments which provide more sensitive methods for the investigation of secondary metabolites. The use of HPLC and GC coupled with mass spectrometry has been proved to be a powerful and popular tool for the investigation of chemical composition (Fischedick et al., 2010; Heinrich et al., 2013; Döll et al., 2013). Metabolic profiling is one of the analytical approaches used to identify different compounds produced in an organism or a group of organisms. Non-targeted metabolic profiling could detect overall consequences, including the differences of metabolism in different species and some genic changed genus (Hanhineva et al., 2009). Therefore, the secondary metabolites in the fungal biotic interactions were investigated mainly based on the HPLC-MS metabolic profiling analytical approaches in this work. Tandem mass spectrometry for the analysis of secondary metabolites was also included in this work.

One part of this study was to explore the variation in secondary metabolites produced by different Botrytis isolates using HPLC-MS metabolic profiling technique. The result of the secondary metabolite based classification of tested Botrytis is coordinated with their morphological characteristic. In addition, some species-specific metabolite signals were found by comparison of their metabolic profiles. This study provides 
further support that the chemotaxonomic analysis based metabolic profiling is a useful tool for classification, as well as for identification of species-specific metabolites. This study, to our knowledge, serves as the first report of classification of Botrytis species based on secondary metabolite profiles using mass spectrometry.

The comparison of the metabolite profiles between $B$. cinerea wild-type B05.10 and its three mutants show significant differences between wild-type B05.10 and its BOT2 mutants while pks6 mutants had the same metabolic profile data as the wild-type B05.10. This suggests that a meaningful comparison of fungal growth among B. cinerea strains should be carried out carefully including all growth phases and physiological stages. Surprisingly, we found that the fungal growth rate of the botrydial mutants B05.10pks6bot2 and B05.10bot2 were slower than wild-type strain B05.10. One recent report suggested that botrydial and botcinic acid might play an unexpected redundant role in saprophytic growth (Dalmais et al., 2011). Our results are similar and we agreed with their suggestion that the phytotoxin botrydial might affect saprophytic growth.

In addition, data-dependent fragmentation (TurboDDS, Varian) which automatically performs $\mathrm{MS}^{\mathrm{n}}$ analysis on selected signals was used for Botrytis species metabolic profiling. Our results suggest that the use of TurboDDS could be a useful way to confirm the structure of the known or unknown compounds in the metabolomics approaches, and the MSMS data provid more reliable information for metabolic analysis. The combination of non-targeted metabolic profiling in full scan mode and the turbo DDS scanning could be a powerful tool for metabolite identification in a metabolic approach.

Furthermore, non-targeted metabolic profiling generated by HPLC-MS was used for monitoring changes of secreted metabolites in fungal co-cultures compared with single cultures. Our results proved that HPLC-MS metabolic profiling method is an effective tool for the investigation of secondary metabolites in fungal interactions.

Several reports of the LC-MS based metabolic profiling for the chemotaxonomy of fungal strains and plants have been published recently (Kang et al., 2011; Kim et al., 2012; Lee et al., 2013; Park et al., 2013). Our results confirmed that the non-targeted 
secondary metabolite-based profiling is an effective tool for classifying Botrytis strains and selecting functional specific metabolites from the diverse metabolite data. In addition, the non-targeted metabolic profiling method is an efficient method for monitoring changes of secreted metabolites from the different fungal cultures. The use of HPLC-MS metabolic profiling played an important role for the secondary metabolite investigation in this study. The advantage of non-targegted metabolic profiling is that it possesses the ability to detect unexpected and unidentified metabolites (Fiehn et al., 2000). Besides this, the $\mathrm{MS}^{\mathrm{n}}$ fragmentation generated by data dependent scanning could be a useful way to confirm the structure of compounds in the metabolomics approaches.

\section{References}

Baek, S.H, Li, A.H., Sassetti, C.M., 2011. Metabolic regulation of mycobacterial growth and antibiotic sensitivity. PLoS Biol. 9(5): e1001065, 1-10.

Bertrand, S., Schumpp, O., Bohni, N., Monod, M., Gindro, K., Wolfender, J.L., 2013. De novo production of metabolites by fungal co-culture of Trichophyton rubrum and Bionectria ochroleuca. J. Nat. Prod. 76, 1157-1165.

Brown, D.W., Butchko, R.A.E., Busman, M., Proctor, R.H., 2012. Identification of gene clusters associated with fusaric acid, fusarin, and perithecial pigment production in Fusarium verticillioides. Fungal Genet. Biol. 49, 521-532.

Crutcher, F., Liu, J., Puckhaber, L., Stipanovic, R., Duke, S., Bell, A., Williams, H., Nichols, R., 2014. Conversion of fusaric acid to fusarinol by Aspergillus tubingensis: a detoxification reaction. J. Chem. Ecol. 40, 84-89.

Cueto, M., Jensen, P.R., Kauffman, C., Fenical, W., Lobkovsky, E., Clardy, J., 2001. Pestalone, a new antibiotic produced by a marine fungus in response to bacterial challenge. J. Nat. Prod. 64, 1444-1446.

Damlmais, B., Schumacher, J., Moraga, J., Le Pecheur, P., Tudzynski, B., Collado, I.G., Viaud, M., 2011. The Botrytis cinerea phytotoxin botcinic acid requires 
two polyketide synthases for production and has a redundant role in virulence with botrydial. Mol. Plant Pathol. 12, 564-579.

Degenkolb, T., Heinze, S., Schlegel, B., Strobel, G., Grafe, U., 2002. Formation of new lipoaminopeptides, acremostatins $\mathrm{A}, \mathrm{B}$, and $\mathrm{C}$, by co-cultivation of Acremonium sp.Tbp-5 and Mycogone rosea DSM 12973. Biosci. Biotechnol. Biochem., 66 (4), 883-886.

Döll, K., Chatterjee, S., Scheu, S., Karlovsky, P., Rohlfs, M., 2013. Fungal metabolic plasticity and sexual development mediate induced resistance to arthropod fungivory. Proc. R. Soc. B Biol. Sci. 280.

Dong, J.Y., Chen, Y.G., Song, H.C., Zhu, Y.H., Zhou, Y.P., Li, L., He, Y.P., Cao, J., Zhang, K.Q., 2007. Hydroxylation of the triterpenoid nigranoic acid by the fungus Gliocladium roseum YMF1.00133. Chem. Biodivers. 4, 112-117.

Fakhouri, W., Walker, F., Armbruster, W., Buchenauer, H., 2003. Detoxification of fusaric acid by a nonpathogenic Colletotrichum sp. Physiol. Mol. Plant Pathol. 63, 263-269.

Fiehn, O., Kopka, J., Dormann, P., Altmann, T., Trethewey, R.N., Willmitzer, L., 2000. Metabolite profiling for plant functional genomics. Nat Biotech 18, 11571161.

Fischedick, J.T., Hazekamp, A., Erkelens, T., Choi, Y.H., Verpoorte, R., 2010. Metabolic fingerprinting of Cannabis sativa L., cannabinoids and terpenoids for chemotaxonomic and drug standardization purposes. Phytochemistry 71, 2058-2073.

García-Granados, A., Gutiérrez, M.C., Parra, A., Rivas, F., 2002. Chemical-microbiological synthesis of cryptomeridiol derivatives by Gliocladium roseum: semisynthesis of 11-hydroxyeudesmanolides. J. Nat. Prod. 65, 1011-1015.

García-Granados, A., Gutiérrez, M.C., Rivas, F., Arias, J.M., 2001. Biotransformation of 4 4 -hydroxyeudesmane-1,6-dione by Gliocladium roseum and Exserohilum halodes. Phytochemistry 58, 891-895.

Hanhineva, K., Kokko, H., Siljanen, H., Rogachev, I., Aharoni, A., Kärenlampi, S.O., 
2009. Stilbene synthase gene transfer caused alterations in the phenylpropanoid metabolism of transgenic strawberry (Fragaria ananassa). J. Exp. Bot. 60, 2093-2106.

Heinrich, J., Valentova, K., Vacek, J., Palikova, I., Zatloukalova, M., Kosina, P., Ulrichova, J., Vrbkova, J., Simanek, V., 2013. Metabolic profiling of phenolic acids and oxidative stress markers after consumption of Lonicera caerulea $L$. fruit. J. Agric. Food Chem. 61, 4526-4532.

Kang, D., Kim, J., Choi, J.N., Liu, K.H., Lee, C.H., 2011. Chemotaxonomy of Trichoderma spp. using mass spectrometry-based metabolite profiling. J. Microbiol. Biotechnol. 21(1), 5-13.

Kim, Y., Lee, J., Park, S.H., Lee, C., Lee, J., Lee, D., Kim, N., Lee, D., Kim, H., Lee, C., 2012. LC-MS-based chemotaxonomic classification of wild-type Lespedeza sp. and its correlation with genotype. Plant Cell Rep. 31, 20852097.

Lee, M.Y., Park, H.M., Son, G.H., and Lee, C.H., 2013. Liquid chromatography-mass spectrometry-based chemotaxonomic classification of Aspergillus spp. and evaluation of the biological activity of its unique metabolite, neosartorin. J. Microbiol. Biotechnol. 23(7), 932 - 941.

Li, C., Zhang, J., Shao, C., Ding, W., She, Z., Lin, Y., 2011. A new xanthone derivative from the co-culture broth of two marine fungi (strain No. E33 and K38). Chem. Nat. Compd. 47, 382-384.

Marín, S., Albareda, X., Ramos, A.J., Sanchis, V., 2001. Impact of environment and interactions of Fusarium verticillioides and Fusarium proliferatum with Aspergillus parasiticus on fumonisin B1 and aflatoxins on maize grain. J. Science of Food and Agriculture 81, 1060-1068.

Oh, D.C., Kauffman, C.A., Jensen, P.R., Fenical, W., 2007. Induced production of Emericellamides A and B from the marine-derived fungus Emericella sp. in competing co-culture. J. Nat. Prod. 70, 515-520.

Park, H.B., Kwon, H.C., Lee, C.H., Yang, H.O., 2009. Glionitrin A, an antibiotic-antitumor metabolite derived from competitive interaction between 
abandoned mine microbes. J. Nat. Prod. 72, 248-252.

Park, S.Y., Lim, S.H., Ha, S.H., Yeo, Y., Park, W.T., Kwon, D.Y., Park, S.U., Kim, J.K., 2013. Metabolite profiling approach reveals the interface of primary and secondary metabolism in colored cauliflowers (Brassica oleracea L. ssp. botrytis). J. Agric. Food Chem. 61, 6999-7007.

Pettit, R., 2009. Mixed fermentation for natural product drug discovery. Appl. Microbiol. Biotechnol. 83, 19-25.

Rodriguez Estrada, A.E., Hegeman, A., Corby Kistler, H., May, G., 2011. In vitro interactions between Fusarium verticillioides and Ustilago maydis through real-time PCR and metabolic profiling. Fungal Genet. Biol. 48, 874-885.

Scherlach, K., Hertweck, C., 2009. Triggering cryptic natural product biosynthesis in microorganisms. Org. Biomol. Chem. 7, 1753-1760.

Schroeckh, V., Scherlach, K., Nützmann, H.W., Shelest, E., Schmidt-Heck, W., Schuemann, J., Martin, K., Hertweck, C., Brakhage, A.A., 2009. Intimate bacterial-fungal interaction triggers biosynthesis of archetypal polyketides in Aspergillus nidulans. Proc. Natl. Acad. Sci. 106, 14558-14563.

Utsumi, R., Hadama, T., Noda, M., Toyoda, H., Hashimoto, H., Ohuchi, S., 1988. Cloning of fusaric acid-detoxifying gene from Cladosporium werneckii: a new strategy for the prevention of plant diseases. J. Biotechnol. 8, 311-316.

Velluti, A., Marín, S., Bettucci, L., Ramos, A.J., Sanchis, V., 2000. The effect of fungal competition on colonization of maize grain by Fusarium moniliforme, $F$. proliferatum and $F$. graminearum and on fumonisin B1 and zearalenone formation. J. Food Microbiol. 59, 59-66.

Zuck, K.M., Shipley, S., Newman, D.J., 2011. Induced production of N-formyl alkaloids from Aspergillus fumigatus by co-culture with Streptomyces peucetius. J. Nat. Prod. 74, 1653-1657. 


\section{Summary}

Fungi are known for their production of chemical and biological diversity of secondary metabolites. To understand the structure, biological activity and function of fungal secondary metabolites contribute to the development of natural product chemistry and mycology. The aim of this study was to investigate the fungal secondary metabolites involved in different fungal interactions.

In this work, HPLC-MS based metabolic profiling was used to study the induction, inhibition and degradation of secreted fungal metabolites in the co-culture of phytopathogen Fusarium verticillioides with mycoparasite Gliocladium roseum. The results indicated that several metabolites were induced or suppressed in the co-cultures; moreover, specific metabolites which only occurred in the dual cultures were found. Large-scale co-cultures of $F$. verticillioides and G. roseum (80 Liter) were prepared for the isolation of interaction-specific metabolites. After extraction, selected metabolites were purified by using combinations of different chromatography methods. Four selected metabolites were obtained from the co-cultures. All of them turned out to be derivatives of fusaric acid (FA). Two of the metabolites (compound 2 and 3) have not been described before.

In addition, the production of known mycotoxins in dual cultures was studied. The amount of fumonisins and FA were decreased in dual cultures with $G$. roseum as compared to $F$. verticillioides single cultures. The comparison of fungal biomass of F. verticillioides in single and co-cultures showed that after 20 days the growth of F. verticillioides was suppressed by G. roseum.

Two experiments were carried out to identify the origin of the FA derivatives. Firstly, both $F$. verticillioides and G. roseum were co-cultivated with other ten different fungal species. Secondly, biotransformation of FA by G. roseum was studied. The results showed that all four FA derivatives only produced in dual cultures of $F$. verticillioides with G. roseum. The two new FA derivatives were biotransformation products of FA 
by G. roseum. Furthermore, we synthesized all the isolated metabolites to test their biological activities against model fungi Botrytis cinerea and Aspergillus niger. The bioassay indicated that the FA derivatives were less toxic than FA; compound 2 was the least toxic FA derivative. We thus showed that the detoxification of FA is involved in fungal interactions.

Another part of this study was on the metabolic profiling of Botrytis species. Metabolic profiling helps to understand how differences in the secondary metabolites among different fungal strains and explain differences in their biological properties. Ten different Botrytis strains, including B. cinerea, B. pseudocinerea, B. fabae and B. group Sa, were incubated for 3 days in Gamborg's B5 medium. After 3 days, the culture supernatants were analyzed by HPLC-MS with full-scan detection and data dependent fragmentation (TurboDDS). Totally 50 metabolic signals were detected and made the comparison of the metabolites such as species-specific or species-nonspecific metabolites, which were used to (i) explore variation in secondary metabolites produced by isolates of Botrytis spp. specialized to different host plants, and (ii) identify changes in metabolic profiles caused by disruption of selected genes of secondary metabolism synthesis. Similarities among aligned metabolic profiles for all tested strains were investigated by hierarchical cluster analysis (HCA) and non-metric multidimensional scaling (MDS). The results support the classification of tested Botrytis strains based on their morphological characteristic and indicate that Botrytis group Sa (D08_H_8 and G09_S33) and B. cinerea were closely related based on their metabolite profiles. The slightly slower growth rate of $B$. cinerea B05.10pks6bot2 and B. cinerea B05.10bot2 than the wild-type B. cinerea B05.10, suggest the phytotoxin botrydial might cause effect in saprophytic growth. Turbo data dependent scanning (TurboDDS) was used for identification of secondary metabolites. Some botrydial-related metabolites were identified by the comparison with the published tandem mass spectrometry data. 


\section{Acknowledgements}

Everyone helped and supported me during my stay in Goettingen, I am thankful to all of you. This thesis would not be finish without any of your help.

First, I would like to acknowledge Prof. Dr. Petr Karlovsky for his guidance and unending support during the last four years. I appreciate his suggestions and discussions throughout this work. It has been a great honor for me to work under his supervision.

Furthermore, I acknowledge Prof. Dr. Matthias Hahn for agreeing to be second supervisor of this work and sincerely appreciate his patient guidance. I am also very grateful to PD. Dr. Micheal Hoppert as an examiner.

I am thankful to the Chinese Scholarship Council (CSC) for the financial support during my study in Germany.

I would like to thank Dr. Subhanker Chatterjee, Dr. Richard Splivallo, Dr. Philip Koessler and Dr. Katharina Doell for their help, guidance and encouragement from the beginning of my work in the lab. I thank PD. Dr. Franze Hadazeck and Dr. Katharina Doell for check of my thesis.

I acknowledge Dr. Kirstin Scherlach and her group (group leader: Prof. Dr. Christian Herweck, Leibniz institute for natural product research and infection biology, Jena, Germany) for their collaboration and help of NMR measurements.

I am thankful to my colleagues and all my institute members, especially Heike Rollwage and Ruth Pilot for their technical support. I thank to Jacqueline Moser and Christian for their technical assistance. I thank to $\mathrm{PhD}$ students and colleagues, in particular to Husam and Raana who consider me as their sister, Patricia for our cooking and dancing together, Eva and Nadine for your introducing of different culture to me. Thanks for the enjoyable time.

I also would like to thank all my Chinese friends. Our friendship makes my life colorful. 
My deepest thanks go to my parents and my grandparents for their support, understanding and the endless love.

I am grateful to my beloved husband, Dr. Shengxiang Yang who supported my study in Germany and tolerated all my bad moods. Thank you for being here in my life. 


\section{Curriculum vitae}

\section{Personal data}

Name:

Yi Kuang

Date of birth:

10.07.1985

Place of birth:

Xi’an, China

\section{Academic education}

Sep 2010 - May 2014 Doctoral research at the Department of Crop Science, Molecular Plant Pathology and Mycotoxin Research Unit, Georg August University Goettingen, Germany

Sep 2007 - Jun 2010 Master of Science, College of Science, Northwest A\&F University, China

Master's thesis "Studies on Alkaloids and Their Bioactivities from the Root of Stephania dolichopoda"

Sep 2003 - Jun 2007

Bachelor of Engine, Institute of Chemical Technology, Xi'an Polytechnic University, China 


\section{Publications}

\section{Publication of the PhD thesis}

Kuang, Y., Chatterjee, S., Scherlach, K., Splivallo, R., Hertweck, C., Karlovsky, P., 2013. Chemical interaction between Fusarium verticillioides and Gliocladium roseum involving mycotoxin transformation. $35^{\text {th }}$ Mycotoxin workshop, Ghent, Belgium (oral presentation).

\section{Other publications}

Li, Y., Ma, Y.T., Kuang, Y., Gao, J.M., Qin, J.C., 2010. Pecipamide, a new sphingosine derivative from the cultures of Polyporus picipes (Basidiomycetes). Lipids 45, 457-461.

Yang, S.X., Wang, H.P., Gao, J.M., Zhang, Q., Laatsch, H., Kuang, Y., 2012. Fusaroside, a unique glycolipid from Fusarium sp., an endophytic fungus isolated from Melia azedarach. Org. Biomol. Chem. 10, 819-824. 